\title{
Extrasolar planets and brown dwarfs around AF-type stars
}

\section{$X$. The SOPHIE sample: combining the SOPHIE and HARPS surveys to compute the close giant planet mass-period distribution around AF-type stars $\star, \star \star$}

\author{
S. Borgniet ${ }^{1}$, A.-M. Lagrange ${ }^{1}$, N. Meunier ${ }^{1}$, F. Galland ${ }^{1}$, L. Arnold ${ }^{2}$, N. Astudillo-Defru ${ }^{3}$, J.-L. Beuzit ${ }^{1}$, \\ I. Boisse ${ }^{4}$, X. Bonfils ${ }^{1}$, F. Bouchy ${ }^{4,5}$, K. Debondt ${ }^{1}$, M. Deleuil ${ }^{4}$, X. Delfosse ${ }^{1}$, M. Desort ${ }^{1}$, R. F. Díaz ${ }^{5}$, \\ A. Eggenberger ${ }^{5}$, D. Ehrenreich ${ }^{5}$, T. Forveille ${ }^{1}$, G. Hébrard ${ }^{2,6}$, B. Loeillet ${ }^{6}$, C. Lovis ${ }^{5}$, G. Montagnier ${ }^{2,6}$, \\ C. Moutou $^{4,7}$, F. Pepe ${ }^{5}$, C. Perrier ${ }^{1}$, F. Pont ${ }^{5}$, D. Queloz ${ }^{5,8}$, A. Santerne ${ }^{4}$, N. C. Santos ${ }^{9,10}$, D. Ségransan ${ }^{5}$, \\ R. da Silva ${ }^{11,12}$, J. P. Sivan $^{2}$, S. Udry ${ }^{5}$, and A. Vidal-Madjar ${ }^{6}$ \\ ${ }^{1}$ CNRS, IPAG, Université Grenoble Alpes, 38000 Grenoble, France \\ e-mail: simon. borgniet@obspm.fr \\ ${ }^{2}$ Observatoire de Haute-Provence, Institut Pythéas UMS 3470, CNRS, Aix-Marseille Université, 04870 St-Michel-1'Observatoire, \\ France \\ ${ }^{3}$ Departamento de Astronomía, Universidad de Concepción, Casilla 160-C, Concepción, Chile \\ ${ }^{4}$ LAM (Laboratoire d'Astrophysique de Marseille) UMR 7326, CNRS, Aix Marseille Université, 13388 Marseille, France \\ ${ }^{5}$ Observatoire Astronomique de l'Université de Genève, 51 Chemin des Maillettes, 1290 Versoix, Switzerland \\ ${ }^{6}$ Institut d'Astrophysique de Paris, UMR 7095 CNRS, Université Pierre \& Marie Curie, 98 bis Boulevard Arago, 75014 Paris, \\ France \\ ${ }^{7}$ Canada-France-Hawaii Telescope Corporation, 65-1238 Mamalahoa Hwy, Kamuela, HI 96743, USA \\ ${ }^{8}$ Cavendish Laboratory, J J Thomson Avenue, Cambridge CB3 0HE, UK \\ ${ }^{9}$ Instituto de Astrofísica e Ciências do Espaço, Universidade do Porto, CAUP, Rua das Estrelas, 4150-762 Porto, Portugal \\ ${ }^{10}$ Departamento de Física e Astronomia, Faculdade de Ciências, Universidade do Porto, Rua do Campo Alegre, $4169-007$ Porto, \\ Portugal \\ 11 ASI - Space Science Data Center (SSDC), Via del Politecnico snc, 00133 Rome, Italy \\ 12 INAF - Osservatorio Astronomico di Roma, Via Frascati 33, 00078 Monte Porzio Catone, Rome, Italy
}

Received 15 May 2018 / Accepted 30 August 2018

\begin{abstract}
Context. The impact of stellar mass on the properties of giant planets is still not fully understood. Main-sequence (MS) stars more massive than the Sun remain relatively unexplored in radial velocity (RV) surveys, due to their characteristics which hinder classical RV measurements.

Aims. Our aim is to characterize the close (up to $\sim 2 \mathrm{au}$ ) giant planet (GP) and brown dwarf (BD) population around AF MS stars and compare this population to stars with different masses.

Methods. We used the SOPHIE spectrograph located on the $1.93 \mathrm{~m}$ telescope at Observatoire de Haute-Provence to observe 125 northern, MS AF dwarfs. We used our dedicated SAFIR software to compute the RV and other spectroscopic observables. We characterized the detected substellar companions and computed the GP and BD occurrence rates combining the present SOPHIE survey and a similar HARPS survey.

Results. We present new data on two known planetary systems around the F5-6V dwarfs HD 16232 and HD 113337. For the latter, we report an additional RV variation that might be induced by a second GP on a wider orbit. We also report the detection of 15 binaries or massive substellar companions with high-amplitude RV variations or long-term RV trends. Based on 225 targets observed with SOPHIE and/or HARPS, we constrain the BD frequency within 2-3 au around AF stars to be below 4\% ( $1 \sigma$ ). For Jupiter-mass GPs within 2-3 au (periods $\leq 10^{3}$ days), we find the occurrence rate to be $3.7_{-1}^{+3} \%$ around AF stars with masses $<1.5 M_{\odot}$, and to be $\leq 6 \%(1 \sigma)$ around AF stars with masses $>1.5 M_{\odot}$. For periods shorter than 10 days, we find the GP occurrence rate to be below 3 and $4.5 \%(1 \sigma)$, respectively. Our results are compatible with the GP frequency reported around FGK dwarfs and are compatible with a possible increase in GP orbital periods with stellar mass as predicted by formation models.
\end{abstract}

Key words. techniques: radial velocities - stars: early-type - planetary systems - stars: variables: general

\footnotetext{
* Based in part on observations made at Observatoire de Haute Provence (CNRS), France.

${ }^{\star \star} \mathrm{RV}$ time series of the full sample are only available at the CDS via anonymous ftp to cdsarc.u-strasbg.fr (130.79.128.5) or via http://cdsarc.u-strasbg.fr/viz-bin/qcat?J/A+A/621/A87
} 


\section{Introduction}

More than three thousand exoplanets and brown dwarfs (BDs) have now been confirmed ${ }^{1}$, while thousands of other Kepler candidates await confirmation. Most of these substellar companions are close $(\leq 5-10 \mathrm{au})$ to their host star and have been detected via radial velocity (RV) and transit surveys (see e.g. Borucki et al. 2010; Howard et al. 2011; Fressin et al. 2013; Mayor et al. 2014). Giant gaseous planets are at the core of the planetary systems as they carry most of their mass. These close giant planets (GPs) have revealed an unexpected diversity of orbital (period, eccentricity, inclination) and physical (mass, composition) properties. This variety emphasizes the complexity of the different processes and interactions that shape the GP population in terms of formation, migration, dynamical interactions, or influence of the primary host star.

While our understanding of the GP formation and evolution mechanisms has dramatically improved over the past twenty years, the influence of stellar properties on GP distribution remains a key topic. For example, it has been clearly established that the GP frequency increases with stellar metallicity for solar-like, main-sequence (MS) FGK dwarfs (Santos et al. 2004; Fischer \& Valenti 2005).

However, the impact of the stellar host mass on its companion's properties is still not fully understood. The core-accretion formation process, which is believed to be at the origin of most of the RV and transit planets, predicts an increase in GP frequency with stellar mass (up to 3-3.5 $M_{\odot}$, Kennedy \& Kenyon 2008). This was confirmed by comparing the GP occurrence rates derived from RV surveys of low-mass M dwarfs (see e.g. Bonfils et al. 2013) and of solar-mass FGK dwarfs (see e.g. Cumming et al. 2008). Subgiant and giant stars seem to confirm this trend for higher stellar masses, as higher GP occurrence rates than those found around solar-mass dwarfs have been reported for these evolved stars (see e.g. Johnson et al. 2010; Reffert et al. 2015). However, the actual mass and origin of these evolved stars is subject to an ongoing controversy, as several studies argue that these giants do not significantly differ from solar-type stars in terms of mass, and are consequently their descendants (instead of being the descendants of AF MS stars; see the list of references in Borgniet et al. 2017).

AF stars with well-established masses above $1 M_{\odot}$ on the main sequence are generally rejected from RV surveys because their specific characteristics (fewer spectral lines and faster rotation) hinder classical RV measurements. A recent survey has targeted chemically peculiar Ap stars, which exhibit lower rotational velocities and more narrow spectral lines than typical AF MS dwarfs (Hartmann \& Hatzes 2015).

Twelve years ago our group developed a tool, Software for the Analysis of the Fourier Interspectrum Radial velocities (SAFIR, Galland et al. 2005b), specifically dedicated to deriving RV measurements of AF MS stars as precisely as possible. For one target, SAFIR cross-correlates each observed spectrum with a reference spectrum built from all the acquired spectra instead of a binary mask, allowing one to reach a better RV precision (closer to the photon noise limit defined by Bouchy et al. 2001). Even if this RV precision remains intrinsically degraded compared to cooler and/or slower rotating stars, we developed SAFIR (and other specific tools) to demonstrate that it is indeed possible to search for close ( $\lesssim 3 \mathrm{au})$ massive $\left(\geq 1 M_{\text {Jup }}\right)$ GP and BD companions around AF MS stars (Galland et al. 2005a, 2006; Lagrange et al. 2009; Meunier et al. 2012).

http://exoplanet. eu, Schneider et al. (2011).
From 2005 to 2016, we carried out a survey targeting GPs and BDs orbiting within $\sim 3$ au around southern AF MS dwarfs with the High-Accuracy Radial velocity Planet Searcher (HARPS, see Pepe et al. 2002) spectrograph on the $3.6 \mathrm{~m}$ ESO telescope at La Silla Observatory in Chile. During this survey, we detected three new GPs around two F5-6V stars (Desort et al. 2008), showed that GPs more massive than Jupiter and BDs are often detectable at periods of up to a thousand days around MS AF dwarfs more massive than the Sun (Lagrange et al. 2009), and derived the first constraints on the occurrence rates of such companions (Borgniet et al. 2017, hereafter Paper IX). Meanwhile, we carried out a survey of northern AF MS stars with the SOPHIE echelle fibre-fed spectrograph (Bouchy \& Sophie Team 2006), which is the successor of the ELODIE spectrograph (Baranne et al. 1996) on the $1.93 \mathrm{~m}$ telescope at Observatoire de Haute-Provence in France. Most of the observations were made in the course of the SP4 programme within the SOPHIE consortium (Bouchy et al. 2009). The first results of our SOPHIE survey, regarding the complex RV variations of HD 185395 and the detection of a $\sim 3 M_{\text {Jup }}$ GP orbiting with a $\sim 320$-day period around HD 113337 , were presented in Desort et al. (2009) and Borgniet et al. (2014), respectively.

This paper first presents our SOPHIE survey and its results, and then presents a statistical analysis combining our SOPHIE and HARPS targets from Paper IX to make a single, global study. The SOPHIE-HARPS combination is possible here because (i) the HARPS and SOPHIE instruments work on the same principles, (ii) they have a similar instrumental RV precision (less than $1 \mathrm{~m} \mathrm{~s}^{-1}$ for HARPS versus a few $\mathrm{m} \mathrm{s}^{-1}$ for SOPHIE), (iii) our surveys have the same overall characteristics, and (iv) we use exactly the same reduction and analysis that we used for our HARPS sample in Paper IX. In Sect. 2, we describe our SOPHIE sample and observations and give a brief outline of our computational tool, our observables, and our RV variability diagnosis. Section 3 is dedicated to the detection and characterization of giant planets and other long-term companions within our SOPHIE survey. In Sect. 4 we make a global analysis of the combined SOPHIE + HARPS sample: (i) we characterize the RV intrinsic variability of our targets, (ii) we derive the search completeness of our combined survey, and (iii) we combine the companion detections and the detection limits to compute the close companion (GP and BD) occurrence rates and estimate their (mass, period) distribution around AF MS stars. We conclude in Sect. 5.

\section{Description of the SOPHIE survey}

\subsection{Sample}

Our SOPHIE sample is made up of 125 AF MS dwarf stars with spectral types in the range A0V to F9V. We detail the physical properties of our sample in Appendix A. We selected our targets using the same selection process as for our HARPS survey (see details in Lagrange et al. 2009, Paper IX and Appendix B). Briefly, our selection relies on the following criteria: (i) the spectral type; (ii) the distance to the Sun, and (iii) the exclusion of known SB2 binary, chemically peculiar stars, or confirmed $\delta$ Scuti or $\gamma$ Doradus pulsators. We ended up with 125 targets with $B-V$ in the range -0.02 to $0.56, v \sin i$ in the range 3 to $250 \mathrm{~km} \mathrm{~s}^{-1}$, and masses in the range 1 to $2.5 M_{\odot}$ (Fig. 1). We note that the exclusion of known pulsators results in a visible dichotomy between our $\sim \mathrm{A}$ - and $\sim$ F-type targets. 

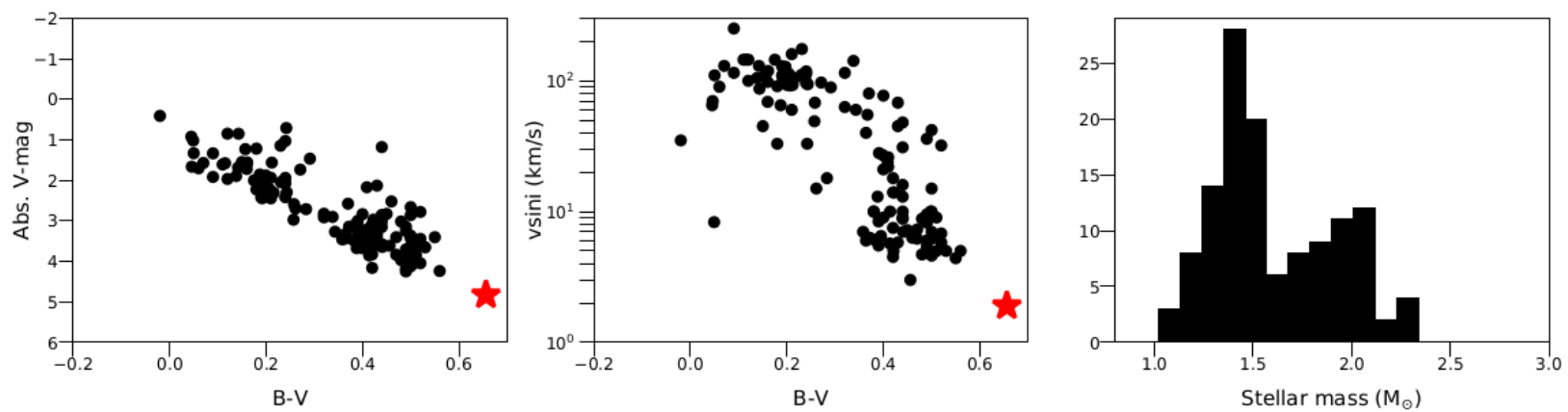

Fig. 1. Main physical properties of our sample. Left panel: HR diagram of our sample, in absolute $V$-magnitude vs $B-V$. Each black dot corresponds to one target. The Sun (red star) is displayed for comparison. Middle panel: $v \sin i$ vs $B-V$ distribution. Right panel: mass histogram of our sample (in $M_{\odot}$ ).
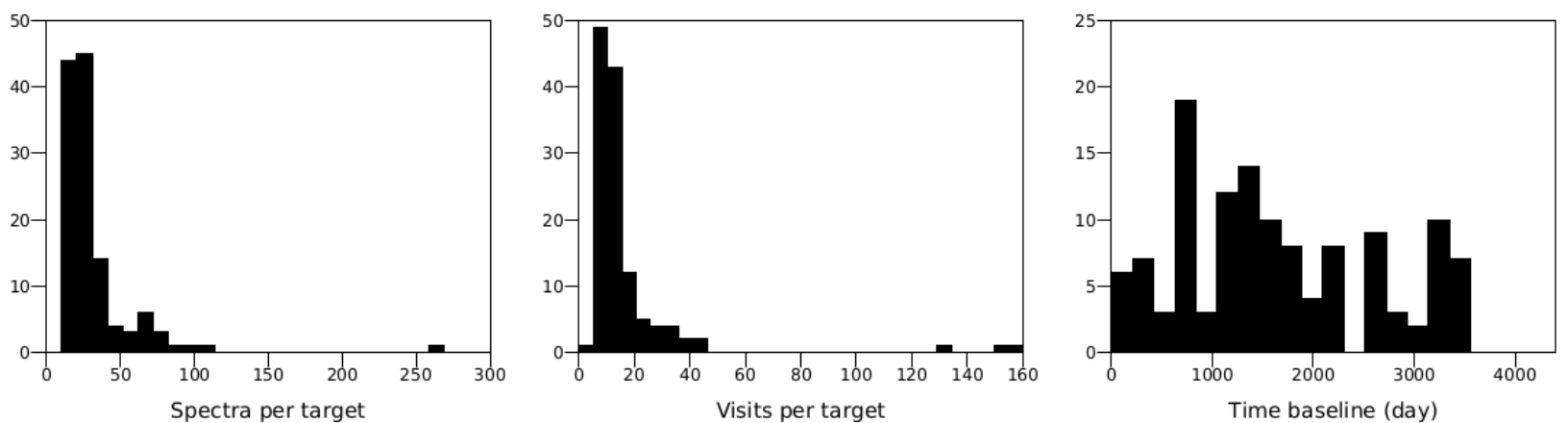

Fig. 2. Observation summary. Left panel: histogram of the spectrum number per target. Middle panel: histogram of the separate observation epoch number per target. Right panel: histogram of the time baselines.

\subsection{Observations}

We observed our 125 SOPHIE targets mainly between November 2006 and April 2014. We made some additional observations in 2015-2016 to complete our survey for the targets with the less data and to further monitor the most interesting targets. We acquired the SOPHIE spectra in high-resolution mode $(R \sim 75000)$, in the 3872-6943 $\AA$ wavelength range. As was done with our HARPS targets, we adapted the exposure times depending on the observing conditions and on the target magnitude in order to get a high signal-to-noise ratio $(\mathrm{S} / \mathrm{N})$, of at least 100 at $\lambda=550 \mathrm{~nm}$. We made most of the exposures in the simultaneousThorium mode, for which the SOPHIE A fibre is centred on the target while the B fibre is fed by a Thorium lamp. This allowed us to follow and correct for a potential instrumental RV drift induced by local temperature or pressure variations, if needed. For very bright targets (very small exposure times), we acquired the spectra in the SOPHIE standard spectroscopy mode (which is appropriate for very bright targets): the A fibre is centred on the target while the B fibre is not illuminated. Our observing strategy and analysis are fully detailed in Paper IX. Briefly, we acquired at least two consecutive spectra at each epoch/visit during several consecutive nights to estimate the short-term stellar jitter of our $\sim$ F-type targets, whereas we acquired multiple consecutive spectra per epoch for our $\sim \mathrm{A}$-type targets, to mitigate the effect of pulsations.

The median time baseline of our SOPHIE survey is 1448 days or $4 \mathrm{yr}$ (the mean time baseline being 1640 days or $4.5 \mathrm{yr}$ ), with a median spectrum number per target of 23 (36 on average) acquired during a median number of 11 visits (17 on average, Fig. 2).

\subsection{Observables}

As was done for our HARPS sample, we computed the RV and, whenever possible, the line profiles and the chromospheric emission in the calcium lines of our targets with our software SAFIR (Chelli 2000; Galland et al. 2005b). The main principles of SAFIR are fully detailed in Paper IX. Our main observables are the RV, the spectrum cross-correlation function (CCF) and its full width at half maximum (FWHM), the bissector velocity span (hereafter BIS; Queloz et al. 2001) and the $\log R_{\mathrm{HK}}^{\prime}$. Our main (but not the only) diagnosis for classifying the RV variability of our targets and distinguishing between its possible sources is the (RV, BIS) diagram; see Paper IX for a fully detailed description of our various observables and of our variability criteria and diagnosis. Very briefly, we classify our targets as RV variable if the total RV amplitude and the RV standard deviation are respectively at least six and two times larger than the mean RV uncertainty. A RV-BIS anti-correlation is most likely the indication of stellar magnetic activity, a RV-BIS vertical diagram indicates pulsations and a flat RV-BIS diagram points toward companionship; CCF distortions and/or RV-FWHM correlation might indicate the presence of a SB2 or SB1 binary. More detailed results on our target observables can be found in Appendix A.

It should be noted that we quadratically add $5 \mathrm{~m} \mathrm{~s}^{-1}$ of systematic error to the SOPHIE uncertainties to take into account the SOPHIE instrumental error (Díaz et al. 2012; Bouchy et al. 2013; Borgniet et al. 2014). Such an error may be slightly excessive for the latest SOPHIE measurements (see below), but we prefer to be conservative and keep the same uncertainty for all our data. 
Table 1. Stellar properties of our targets with detected GPs.

\begin{tabular}{lccc}
\hline \hline Parameter & Unit & HD 16232 & HD 113337 \\
\hline Spectral type & & ${\mathrm{F} 6 V^{a}}^{c}$ & ${\mathrm{~F} 6 V^{b}}^{c}$ \\
$V$ & & $7.09^{c}$ & $6.03^{c}$ \\
$B-V$ & & $0.5^{c}$ & $0.37^{c}$ \\
$v \sin i$ & $\left(\mathrm{~km} \mathrm{~s}^{-1}\right)$ & $30^{c}$ & $6^{c}$ \\
$\pi$ & $(\mathrm{mas})$ & $24.52 \pm 0.68^{d}$ & $27.11 \pm 0.29^{d}$ \\
{$[\mathrm{Fe} / \mathrm{H}]$} & & $-0.03^{e}$ & $0.09^{e}$ \\
$T_{\text {eff }}$ & $(\mathrm{K})$ & $6396 \pm 95^{e}$ & $6669 \pm 80^{e}$ \\
$\log g$ & $(\mathrm{dex})$ & $4.44 \pm 0.08^{f}$ & $4.21 \pm 0.08^{f}$ \\
$M_{\star}$ & $\left(M_{\odot}\right)$ & $1.20 \pm 0.09^{f}$ & $1.41 \pm 0.09^{f}$ \\
Radius & $\left(R_{\odot}\right)$ & $1.10 \pm 0.05^{f}$ & $1.55 \pm 0.07^{f}$ \\
Age & $(\mathrm{Gyr})$ & $0.9 \pm 0.8^{a}$ & $0.15_{-0.05}^{+0.1}{ }^{g}$ \\
\hline
\end{tabular}

References. ${ }^{(a)}$ Guenther et al. (2009). ${ }^{(b)}$ Boesgaard \& Tripicco (1986). ${ }^{(c)}$ Taken from the CDS. The uncertainties on the $v \sin i$ are not provided. ${ }^{(d)}$ van Leeuwen (2007). ${ }^{(e)}$ Casagrande et al. (2011). The catalog does not provide the uncertainties on the metallicity. ${ }^{(f)}$ Allende Prieto \& Lambert (1999). ${ }^{(g)}$ Borgniet et al. (2014).

\subsection{SOPHIE+}

In June 2011 (BJD 2455730), SOPHIE was updated (and called SOPHIE+) by implementing octagonal fibres at the fibre link (Bouchy et al. 2013). Prior to this update, the SOPHIE RV were known to exhibit a systematic bias induced by the insufficient scrambling of the old fibre link. This bias was called the "seeing effect" as seeing variations at the fibre entrance were causing RV variations (Boisse et al. 2011). One way to correct for this bias is to measure the RV in the blue and red halves of each SOPHIE spectral order and compute their difference $\delta_{\mathrm{RV}}$, which should be correlated to the original RV if affected by the seeing effect, as detailed in Díaz et al. (2012). We applied the same correction in SAFIR, computing $\delta_{\mathrm{RV}}$ for all spectra acquired prior to June 2011, and correcting the RV from the $\delta_{\mathrm{RV}}$ correlation if needed (i.e. if the Pearson's correlation coefficient was above 0.5 in absolute value). This happened for 16 of our targets for which the $\delta_{\mathrm{RV}}$ correlation was $>0.5$.

\section{Detected companions in the SOPHIE survey}

\subsection{Giant planets}

In this section we present two planetary systems with three GPs, either confirmed or probable candidates. The host stars' fundamental properties are detailed in Table 1, and the orbital parameters of the detected and/or candidate GPs in Table 2. The $\mathrm{RV}$ and line profile data of these systems are shown in detail in Appendix D.

\subsubsection{HD 113337 system}

Summary of first planet detection. We reported the detection of a giant planet orbiting around HD 113337 and characterized it in Borgniet et al. (2014, hereafter BO14). HD 113337 (HIP 63584) is a F6V star with a $1.41 M_{\odot}$ stellar mass (Allende Prieto \& Lambert 1999), a slight infrared excess attributed to a cold debris disk (Rhee et al. 2007), and may be relatively young (possible age of $150 \pm 100 \mathrm{Myr}$; see BO14). Based on 266 SOPHIE RV measurements spanning six years, we reported clear 320-day periodic RV variations along with a longer term, quadratic-shaped RV drift and a flat (RV, BIS) diagram.
We showed that the periodic RV variations are induced by a $m_{\mathrm{p}} \sin i \sim 2.8 M_{\text {Jup }}$ giant planet orbiting around HD 113337 with a 324-day period on an eccentric orbit $(e \simeq 0.46)$. Based on weak long-term variations observed in the BIS and FWHM, we finally supposed that the long-term quadratic RV variation was more probably induced by a stellar activity cycle than by an additional companion.

New SOPHIE data. As a target of great interest, we continued to observe HD 113337 with SOPHIE in 2014-2016, adding 35 additional spectra to our database and consequently expanding the time baseline by $\sim 3 \mathrm{yr}$, to $9.2 \mathrm{yr}$ (3369 days). We display the main SOPHIE spectroscopic observables for this target in Fig. D.1. Most interestingly, the new RV data exhibits a clear rebound on the long-term scale around Julian Day (hereafter JD) 2457000. It means that the long-term RV variations can no longer be modelled by a quadratic fit and could possibly be periodic. We computed the RV periodograms according to the Lomb-Scargle definition (Scargle 1982; Press \& Rybicki 1989), and also with the CLEAN algorithm (which deconvolves the Lomb-Scargle periodogram from the window function; see Roberts et al. 1987). Both periodograms exhibit two clear peaks (Fig. D.1, top row), one at $\sim 320$ days corresponding to the already-known GP (hereafter HD 113337b), and another at $\sim 3200$ days corresponding to the long-term variations.

The BIS data has a $24.5 \mathrm{~m} \mathrm{~s}^{-1}$ dispersion and does not show any high-amplitude variations. However, a slight longterm variation may be present with a BIS extremum between BJD 2455600 and BJD 2456500, approximately (Fig. D.1, second row). Except for two peaks at $\sim 2$ and $\sim 3$ days which are probably induced by the stellar rotation, the BIS Lomb-Scargle periodogram does not exhibit any significant signal for periods up to 1000 days. However, it shows power between 2000 and 4000 days, i.e. in roughly the same period range as the observed RV long-term variations. When looking at the BIS CLEAN periodogram, the long-term peak is centred at a $\sim 2000$-day periodicity (i.e. quite distinct from the $\sim 3200$-day RV periodicity) and is no longer the highest peak in the periodogram (the highest peak being at $\sim 2.5$ days). The (RV, BIS) diagram is nearly flat (with a $-0.12 \pm 0.02$ linear slope and a Pearson correlation coefficient of -0.32), thus ruling out stellar activity as the only source of the RV variations.

The FWHM time series exhibits a high-amplitude $\left(\sim 250 \mathrm{~m} \mathrm{~s}^{-1}\right)$ long-term variation, with a minimum between BJD 2455700 and BJD 2456200 (Fig. D.1, third row). In the same way as the BIS, the FWHM Lomb-Scargle periodogram exhibits power between 2000 and 4000 days, and the FWHM CLEAN periodogram exhibits a clear, single peak around 2200 days. There is no other significant signal at shorter periods; the peaks present in the Lomb-Scargle periodogram around 200-400 days and at $\sim 30$ days are aliases induced by the temporal sampling (see window function). The Pearson coefficients for the (RV, FWHM) and (BIS, FWHM) couples are 0.25 (no correlation) and -0.49 (anti-correlation), respectively. This suggests a common origin for the long-term (high-amplitude) FWHM and (low-amplitude) BIS variations (possibly an activity cycle) while giving further credence to another origin to the $\mathrm{RV}$ variations.

HD 113337 does not show any chromospheric emission in the calcium $\mathrm{H}$ and $\mathrm{K}$ lines (Borgniet et al. 2014). The $\log R_{\mathrm{HK}}^{\prime}$ has a mean value of -4.76 and does not exhibit any significant signal.

A second GP around $H D$ 113337. Given the new SOPHIE RV data, we tried to fit HD 113337 RV variations with a two-planet Keplerian orbital model (Fig. D.1). 
Table 2. Best orbital solutions.

\begin{tabular}{llccc}
\hline \hline Parameter & Unit & HD 16232b & HD 113337b & HD 113337c \\
\hline Status & & Probable & Confirmed & Possible \\
\hline$P$ & day & $345.4 \pm 3.7$ & $323.4 \pm 0.8$ & $3264.7 \pm 134.3$ \\
$T_{0}$ & BJD-2453000 & $912.8 \pm 42.5$ & $2757.5 \pm 4$ & $5236.8 \pm 217.1$ \\
$e$ & & $0.18 \pm 0.13$ & $0.36 \pm 0.03$ & $0.18 \pm 0.04$ \\
$\omega$ & $\circ$ & $-22.9 \pm 44.1$ & $-130.9 \pm 4.9$ & $-46 \pm 13$ \\
$K$ & $\mathrm{~m} \mathrm{~s}^{-1}$ & $176.6 \pm 31.5$ & $76.1 \pm 2.9$ & $76.7 \pm 2.4$ \\
\hline Lin. & $\mathrm{m} \mathrm{s}^{-1} \mathrm{yr}^{-1}$ & $-44.1 \pm 10.2$ & 0 & - \\
Quad. & $\mathrm{m} \mathrm{s}^{-1} \mathrm{yr}^{-2}$ & 0 & 0 & - \\
$N_{\mathrm{m}}$ & & 110 (from Kane et al. 2015) & 301 & - \\
$\sigma_{\mathrm{O}-\mathrm{C}}$ & $\mathrm{m} \mathrm{s}^{-1}$ & $128(228.4)^{b}$ & $22.3(67.4)^{b}$ & - \\
Reduced $\chi^{2}$ & & $4.4(25.4)^{b}$ & $2.9(8.6)^{b}$ & - \\
\hline$m_{\mathrm{p}} \sin i$ & $M_{\mathrm{Jup}}$ & $6.8 \pm 1.4$ & $3 \pm 0.3$ & $6.9 \pm 0.6$ \\
$a_{P}$ & $\mathrm{au}$ & $1.03 \pm 0.01$ & $1.03 \pm 0.02$ & $4.8 \pm 0.23$ \\
\hline
\end{tabular}

Notes. ${ }^{(a)}$ The orbital parameters for HD 16232b were computed with yorbit based on the RV measurements given by Kane et al. (2015) and the stellar mass value from Allende Prieto \& Lambert (1999). ${ }^{(b)}$ The number in parentheses refers to the model assuming a constant velocity.

To perform the Keplerian fits, we used the yorbit software (Ségransan et al. 2011), as we did in our previous studies (see e.g. BO14 and Paper IX). Our two-planet best solution gives orbital parameters for planet $\mathrm{b}$ that are in agreement with the values that we derived in BO14 in terms of period $(323 \pm 1$ days against $324 \pm 2$ days, respectively) and minimal mass $\left(3 \pm 0.3 M_{\text {Jup }}\right.$ against $2.8 \pm 0.3 M_{\text {Jup }}$, respectively). Interestingly, the eccentricity of planet $\mathrm{b}$ is significantly reduced by going from a one-planet to a two-planet solution (from $0.46 \pm 0.04$ to $0.36 \pm 0.03$, respectively). If considering that the RV long-term variations are induced by a second GP (hereafter planet c), our best model would correspond to a period of $\sim 3265 \pm 134$ days and a $6.9 \pm 0.6 M_{\text {Jup }}$ minimal mass on a slightly eccentric $(e \simeq 0.2)$ orbit. The RV residuals of the two-planet Keplerian fit show a remaining dispersion of $\sim 23 \mathrm{~m} \mathrm{~s}^{-1}$ (Fig. D.1, fifth row), with a significant anti-correlation with the BIS (Pearson coefficient of -0.44). The periodograms of the RV residuals mainly show small peaks at $\sim 2$ and $\sim 4$ days, and at $\sim 90$ days. None of these periodicities can be properly fitted with yorbit. We attribute the short-period peaks to the stellar rotation as they are also present in the BIS periodogram. The origin of the remaining $\sim 90$-day peak is less clear, but a similar peak is present in the BIS periodograms (Fig. D.1, second row). Thus, we do not find any significant sign of a third companioninduced periodicity. The orbital parameters we deduced from the two-planet Keplerian fit are fully detailed in Table 2.

While the long-term RV variation can be modelled well by a Keplerian fit corresponding to a second GP, other possible origins can also be considered, due to the presence of FWHM (and, on a much smaller scale, BIS) long-term variations. We review below the different hypotheses and their pros and cons (second GP included), and explain why we consider the second GP hypothesis to be the most convincing one.

Case of an activity cycle. Magnetic activity cycles are characterized by the rise and fall in the number of magnetically active structures on the stellar photosphere (such as dark spots and bright faculae) over durations much longer than the stellar rotation period. They can induce RV and/or line profile and/or $\log R_{\mathrm{HK}}^{\prime}$ variations. Activity cycles with associated timescales of a few years are relatively common among FGK MS dwarfs (see e.g. Schröder et al. 2013). For example, reconstructed solar RV exhibit a peak-to-peak RV amplitude of $8-10 \mathrm{~m} \mathrm{~s}^{-1}$ over solar cycle 23 (Meunier et al. 2010). Given that HD 113337 $v \sin i$ of $6 \mathrm{~km} \mathrm{~s}^{-1}$ is higher than SOPHIE instrumental resolution $\left(\sim 4 \mathrm{~km} \mathrm{~s}^{-1}\right)$, magnetic stellar activity should induce both RV and line profile (BIS and/or FWHM) variations (see e.g. Desort et al. 2007; Lagrange et al. 2009, and Paper IX).

In this hypothesis, an activity cycle with a duration approximating that of the candidate planet $\mathrm{c}$ period would explain both the FWHM and BIS long-term variations (and their correlation) as well as the RV long-term variations. We consider though that this interpretation has an important weakness: the RV corrected from the Keplerian fit of planet b only and the FWHM are not correlated (Pearson's coefficient $<0.1$, see Figs. D.1 and 3). This is due to the RV and FWHM long-term variations being significantly shifted in phase (considering a period of $~ 3265$ days from the two-planet Keplerian fit). A phase shift of 15 to $30^{\circ}$ between RV, BIS, and FWHM activity-induced variations can be expected in the case of short-term stellar activity linked to the stellar rotational period (see e.g. Santos et al. 2014; Dumusque et al. 2014, who show that an active region crossing the visible stellar disk induces a RV shift and a line profile deformation with a slight phase shift). However, such a phase shift cannot be present in the case of a long-term activity cycle where the RV and line profile long-term variations are induced by the changing active region filling factor of the stellar disk along the cycle (Borgniet et al. 2015), being thus correlated (Dumusque et al. 2011). In the case of HD 113337, we derived the Pearson's coefficient of the $\mathrm{RV}$ residuals of planet $\mathrm{b}$ versus FWHM while shifting in phase the FWHM along the $~ 3265$-day period deduced from the twoplanet Keplerian fit (Fig. 3). The highest correlation $(\sim 0.65)$ is reached for a phase shift of $\sim 0.45$ (or 1470 days), while the highest anti-correlation (approximately -0.65 ) is reached for a phase shift of $\sim 0.7$ (or 2290 days). While we still do not understand everything about stellar magnetic activity cycles, we consider that the observed shift between the RV and FWHM long-term variations is a strong argument against the activity cycle origin for the RV long-term variations, given our present knowledge (Dumusque et al. 2011). We note that long-period RV planets have already been distinguished from activity cycles by looking for the presence of a phase shift between the RV and the $\log R_{\mathrm{HK}}^{\prime}$ taken as an activity proxy (Wright 2016).

Case of a faint binary. A faint, low-mass stellar companion to HD 113337 in a (nearly) pole-on configuration could 

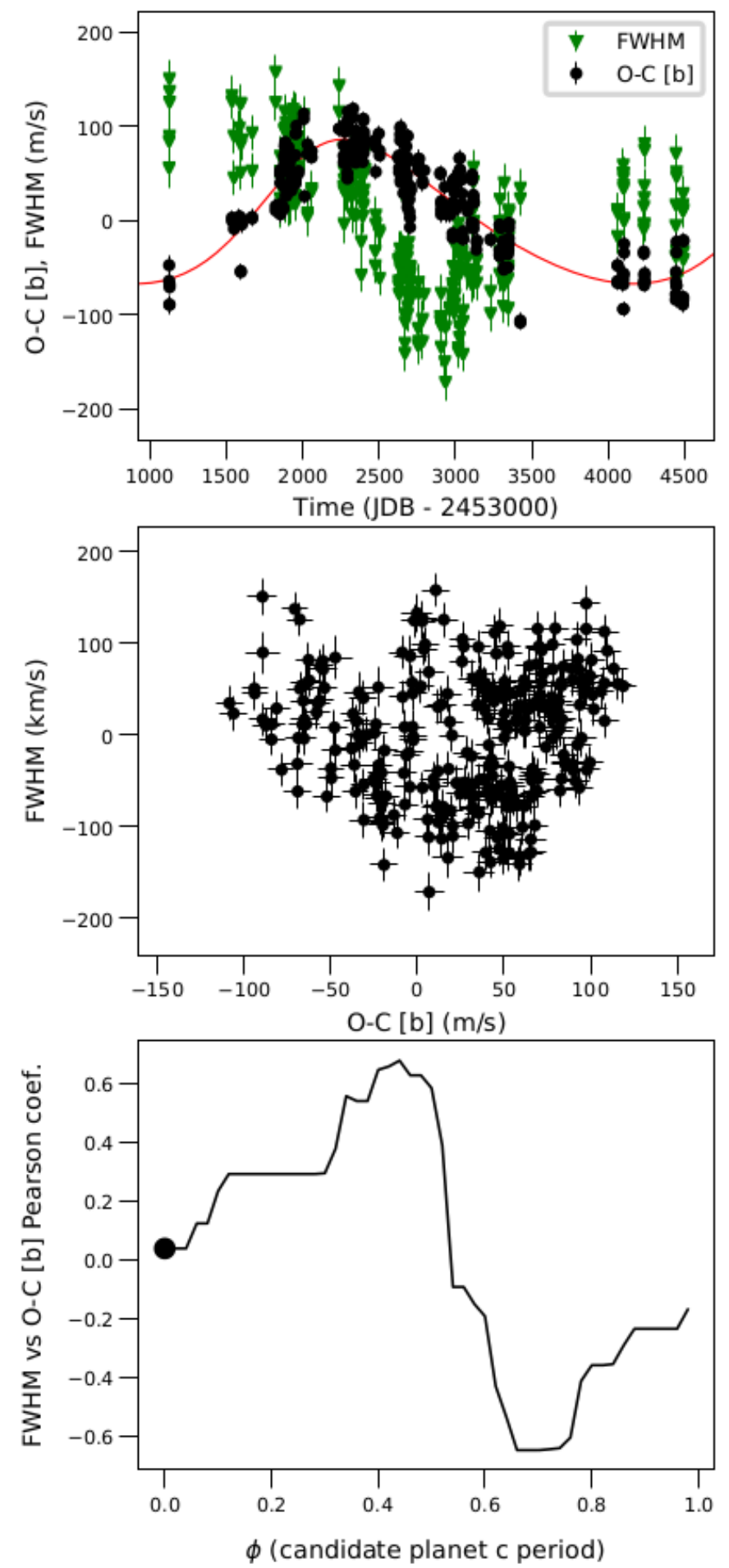

Fig. 3. Top panel: HD 113337 RV residuals from the Keplerian fit of planet b only vs time (black dots) and HD 113337 FWHM vs time (green triangles, same scale as RV). The Keplerian fit of candidate planet $\mathrm{c}$ is overplotted to the RV residuals of planet $b$ (red). The average FWHM $\left(\sim 13.7 \mathrm{~km} \mathrm{~s}^{-1}\right)$ has been set to 0 for clarity. Middle panel: FWHM vs RV residuals of planet b. Bottom panel: Pearson's correlation coefficient of RV residuals of planet b vs FWHM for different FWHM phase shifts, considering a $\sim 3265$-day period (black solid line). The actual Pearson's coefficient (for no FWHM phase shift) is displayed as a black dot.

be just bright enough to slightly blend the primary spectrum, and thus induce the long-term FWHM and (low-amplitude) RV variations. HD $113337 v \sin i$ is quite low for a F6V spectral type (see Borgniet et al. 2014), meaning that the system could be seen inclined (assuming that the star rotates in the same plane as the planetary system). Furthermore, HD 113337 is host to a cold debris disk that is most likely also inclined (K. Su, priv. comm.). However, in such a configuration, the $\mathrm{RV}$ and FWHM companion-induced variations would also have to be correlated (see Sect. 3.3.11), which is not the case here. Moreover, given the $\sim 7 M_{\text {Jup }}$ minimal mass of the second candidate companion, the system inclination would have to be lower than $3^{\circ}$ to allow for an actual companion mass of $\sim 150 M_{\text {Jup }}$, which makes it quite improbable statistically. Stellar mass-luminosity relations (Baraffe et al. 1998) taken for an age between 120 and $500 \mathrm{Myr}$ show that a companion mass of $150 M_{\mathrm{Jup}}$ would translate into a contrast of 6 magnitudes with the primary in the $H$ band, and so even greater in the $V$ band. This would make the effect of the companion light on the primary spectrum completely negligible (especially when using a reference built from the primary spectra for the cross-correlation). Thus, we find the hypothesis of a low-mass stellar companion difficult to support given the observations.

Case of planet $c$. The RV and FWHM long-term variations are phase-shifted, which is not coherent with a magnetic activity cycle (see above). Furthermore, if plotting the BIS versus the RV residuals of planet $b$ (Fig. D.1, fourth row), we find a still nearly flat diagram with a linear slope of $-0.14 \pm 0.02$ and a correlation coefficient of -0.29 . These values are very close to those we find for the (RV, BIS) diagram (see above), meaning that the horizontal spread in the (RV, BIS) diagram (characteristic of the presence of a companion) may well be induced by more than one planet. On the contrary, the RV residuals of the two-planet fit plotted versus the BIS show a linear slope of $-0.49 \pm 0.05$ and an enhanced anti-correlation ( -0.44 , see above), meaning that the remaining RV dispersion is likely induced by stellar activity. We consider that the presence of a second GP in the system is thus a very convincing explanation for the RV long-term variations and the shapes of the RV and RV residuals versus BIS diagrams. The additional presence of a long-term activity cycle would then explain the long-term FWHM and BIS variations, and the anti-correlation present between the RV residuals and the BIS.

To conclude here, we decided to classify HD 113337 as a candidate two-planet system (planet $\mathrm{b}$ being confirmed and planet $\mathrm{c}$ still a candidate).

Additional remarks. In addition to the SOPHIE observations, we are carrying out a multi-technique study (optical interferometry, disk imaging, and deep imaging of the outer environment) of the HD 113337 system. The aim of this study is to bring as many constraints as possible on the system characteristics (stellar fundamental parameters, age, inclination, etc.) and to fully cover the companion (mass, separation) space. This will be the topic of a forthcoming, dedicated paper.

\subsubsection{Giant planet around HD 16232}

HD 16232 (30 Ari B, HIP 12184) is a F6V dwarf star around which Guenther et al. (2009) reported the detection of a substellar companion of $\sim 10 M_{\text {Jup }}$ minimal mass, based on RV observations. This companion was further characterized thanks to new RV measurements (for a total of 110 spectra; see Kane et al. 2015, hereafter K15). These authors made a new estimation of the companion's orbital parameters, making it of planetary nature, and additionally detected a long-term linear RV trend likely induced by a distant stellar companion. The 


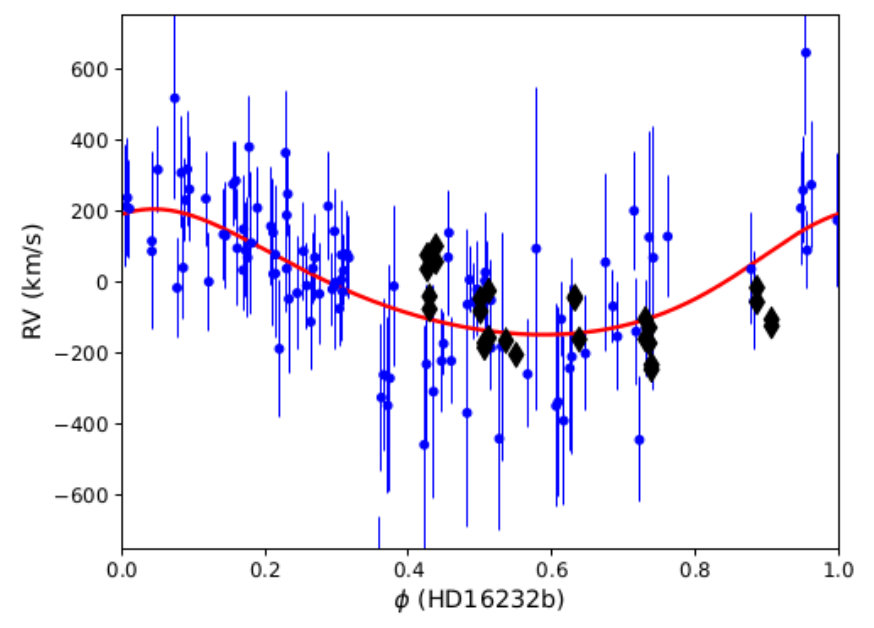

Fig. 4. HD 16232 phased RV. RV from K15 are displayed as blue dots, while our SOPHIE RV are displayed as black diamonds. The data are phased along the orbit of HD $16232 \mathrm{~b}$ according to the Keplerian fit of $\mathrm{K} 15 \mathrm{RV}$ in Table 2 (the corresponding best model is displayed in red).

Keplerian+linear fit performed by K15 thus gives $m_{\mathrm{p}} \sin i=$ $6.6 \pm 0.9 M_{\mathrm{Jup}}, P=345.4 \pm 3.8$ days, and $e=0.18 \pm 0.11$, corresponding to a $1.01 \pm 0.01$ au semi-major axis (sma) for HD $16232 b$.

As part of our SOPHIE survey, we acquired 27 spectra on HD 16232, covering a 1893-day baseline. These data are enough to allow us to clearly see the long-term RV trend first detected by K15 in our SOPHIE RV (Fig. D.2, third row). Furthermore, we find that the SOPHIE BIS versus RV diagram looks composite, with a horizontal spread indicative of a companion and a vertical spread induced by stellar activity and/or low-level pulsations. The SOPHIE BIS and FWHM do not show any significant signal. We characterize the binary companion at the origin of the long-term RV trend in more detail in Sect. 3.3.2. However, we do not detect any signal other than the binary-induced trend in our SOPHIE RV (Fig. D.2, sixth row). This non-detection may be induced by our smaller number of observations compared to K15 and because our GP detectability is significantly degraded by the presence of the additional RV trend induced by the distant stellar companion. When plotting both RV data sets phased along the period from K15 (Fig. 4), we find that our SOPHIE RV and the best model from K15 are not in perfect agreement; however, it is unclear whether this is induced by a phase shift between our data and the fit or by the relatively high stellar noise in the data. If combining both RV data sets with yorbit, we do not achieve a good one-planet Keplerian fit. We note that we obtain a much better RV accuracy than K15 thanks to the use of SOPHIE and SAFIR. The Lomb-Scargle periodogram of the SOPHIE RV is completely dominated by the window function, due to the presence of the RV linear drift. On the contrary, the Lomb-Scargle and CLEAN periodograms of the more consequent K15 RV data set exhibit a clear peak at the planet period.

To investigate the impact of our temporal sampling on HD 16232b detectability, we computed the expected RV given K15 orbital fit at the epochs of our SOPHIE observations. To do so, we extrapolated the Keplerian+linear fit from K15 to our observation epochs, and then added RV white noise with the same dispersion as the short-term (one night) RV jitter we obtained in our SOPHIE RV data. These extrapolated RV look the same as our SOPHIE RV, the RV linear drift being detectable, but not the planet-induced periodic RV variations (Fig. D.2, second row). Moreover, the Lomb-Scargle periodogram of the extrapolated RV look very much the same as for our SOPHIE RV (i.e. it is dominated by the window function), meaning that the GP detected by K15 is most probably non-detectable here, given our temporal sampling, our small number of observations compared to K15, and the level of short-term RV stellar jitter. Additional SOPHIE observations sampled over the orbital period of HD $16232 \mathrm{~b}$ could raise the ambiguity.

In the context of the following statistical analysis, we nonetheless decided to include HD $16232 \mathrm{~b}$ as a strong candidate GP. Based on the Keplerian fit by K15, we computed HD $16232 \mathrm{~b}$ minimal mass considering a stellar mass of $1.2 M_{\odot}$ to be fully consistent with our stellar mass values, finding a slightly increased $m_{\mathrm{p}} \sin i=6.8 \pm 1.4 M_{\text {Jup }}$. We include the detailed parameters of the Keplerian fit in Table 2.

\section{2. $\theta$ Cyg: a system with complex $R V$ variations}

We detail here our results on HD 185395 or $\theta$ Cyg, a target that we observed extensively along our SOPHIE survey and that exhibits intriguingly complex RV and line profile variations. The $\mathrm{RV}$ and line profile data of $\theta$ Cyg are illustrated in detail in Appendix D.

The HD 185395 system. We presented our first results on $\theta$ Cyg $\left(\mathrm{F} 4-5 \mathrm{~V}, 1.37 M_{\odot}\right)$ in Desort et al. (2009, hereafter D09). We made a first analysis of the RV based on 91 ELODIE spectra and our first 162 SOPHIE spectra. We showed in D09 that both ELODIE and SOPHIE data sets exhibit a strong quasi-periodic $\mathrm{RV}$ signal, with a $\sim 220 \mathrm{~m} \mathrm{~s}^{-1}$ amplitude and a main periodicity of $\sim 150$ days. Along with these RV variations was a flat (RV, BIS) diagram, the BIS variations being of much smaller amplitude $\left(\sim 50 \mathrm{~m} \mathrm{~s}^{-1}\right)$ than the RV. We argued in D09 that given the $7 \mathrm{~km} \mathrm{~s}^{-1} v \sin i$ of the target, such a flat (RV, BIS) diagram would usually be a clear sign that the RV variations are induced by a planetary companion and are not of stellar origin. However, we also showed that despite this flat (RV, BIS) diagram, the BIS exhibits a strong periodicity of around 150 days, making the origin of this periodicity puzzling. Furthermore, the $\sim 150$-day RV variations could not be easily fitted with Keplerian models, with no stable and/or satisfying solution. We concluded in D09 that the origin of these complex RV and BIS variations could not yet be assessed. In addition, we reported the detection by imaging of a wide stellar companion to $\theta \mathrm{Cyg}$, that yet could not be responsible for the $\sim 150$-day RV and line profile variations. $\theta$ Cyg was also independently followed in RV by another team (Howard \& Fulton 2016). Based on $223 \mathrm{RV}$ measurements obtained at the Lick Observatory, these authors also detected the long-term RV drift, as well as the quasi-periodic RV variations at $\sim 150$ days, that they deemed statistically non-significant.

Finally, $\theta$ Cyg has been a target of interest in optical and IR interferometry. Multiple VEGA observations allowed Ligi et al. (2012) to make a first estimation of $\theta$ Cyg angular diameter. Furthermore, the unusually high jitter and a possible periodic variability in the squared visibility measurements led the authors to speculate on the presence of a low-mass, unseen close stellar companion as the possible source of the RV and interferometric jitter. However, the more recent study of White et al. (2013), based notably on IR closure phase (CP) measurements made with the Michigan Infrared Combiner (MIRC, Monnier et al. 2004) interferometer on CHARA, found no evidence for a close stellar companion to $\theta \mathrm{Cyg}$. Based on the $\mathrm{CP}$, these authors derived upper limits on a potential companion brightness, and found that a companion would have to be fainter than the primary by at least 4.7 magnitudes in the $H$ band between 0.2 and 0.4 au, and fainter by at least 3.4 magnitudes between 0.4 and 0.7 au. 
Using stellar mass-luminosity relations (Baraffe et al. 1998) for an age of $\sim 1 \mathrm{Gyr}$, this translates into upper masses of 0.3 and $0.5 M_{\odot}$, respectively.

New SOPHIE data. We acquired 164 additional spectra on $\theta$ Cyg from 2009 to 2016, raising the total number of SOPHIE spectra to 326 , and extending the SOPHIE time baseline to 3482 days $(\sim 9.5 \mathrm{yr})$. We display the main SOPHIE spectroscopic observables in Fig. D.3. The new SOPHIE RV data set show both a long-term drift of slightly quadratic shape as well as the quasi-periodic $\sim 150$-day variations. The quadratic trend is probably induced by the wide stellar companion imaged by D09 (see details in Sect. 3.3.10). Once corrected from this quadratic trend, the remaining RV variations have a total amplitude of $275 \mathrm{~m} \mathrm{~s}^{-1}$ and a dispersion of $64.5 \mathrm{~m} \mathrm{~s}^{-1}$. The RV Lomb-Scargle periodogram exhibits several peaks above the 1\% FAP between 100 and 500 days, but only a single peak at 150 days remains when cleaning the periodogram from the window function (CLEAN, Fig. D.3).

HD 185395 BIS shows only low-amplitude variations (rms $12.5 \mathrm{~m} \mathrm{~s}^{-1}$ ), thus leading to a flat (RV, BIS) diagram, indicative of a companion. However, the BIS Lomb-Scargle periodogram exhibits a single peak at a 145-day periodicity (in agreement with D09) which is also present in the CLEAN periodogram. The FWHM shows periodic variations with a $207 \mathrm{~m} \mathrm{~s}^{-1}$ total amplitude (rms $38 \mathrm{~m} \mathrm{~s}^{-1}$ ), corresponding to a single peak at 149 days on the FWHM periodograms. The amount of correlation between the RV corrected from the quadratic fit and the FWHM is low (-0.3), but slightly higher than between the uncorrected RV and the FWHM (-0.2). Both the BIS and FWHM data can be fitted fairly well with a nearly sinusoidal model with yorbit, with periods of 144 and 148 days, respectively, and marginally compatible phases. Finally, we recall that $\theta$ Cyg does not show any chromospheric emission in the calcium lines $\left(\left\langle\log R_{\mathrm{HK}}^{\prime}\right\rangle=-4.82\right)$.

Origin of the $R V$ variations. As already shown by D09, a purely stellar origin to the $\sim 150$-day RV and line profile variations is unlikely. Given $\theta$ Cyg $v \sin i$ of $\sim 7 \mathrm{~km} \mathrm{~s}^{-1}$, the rotational modulation of stellar magnetic activity should induce RV-correlated BIS variability, which is inconsistent with the flat (RV, BIS) diagram. Stellar granulation is not known to induce such high-amplitude RV variations at such a long period, whereas magnetic activity cycles last much longer given our present knowledge (several years, see Baliunas et al. 1995). Finally, most stellar pulsations (i.e. solar-like or $\gamma$ Dor-type) happen at much shorter periodicities; Kepler data have revealed solar-like oscillations on $\theta$ Cyg, but no other clear pulsation pattern (Guzik et al. 2011).

A second hypothesis could be the presence of a close, unseen low-mass stellar companion, which was not explored by D09. Such a companion would be just bright enough to slightly blend the primary spectrum and induce the $\sim 150$-day periodic variation in both the RV and the line profile observables without significantly altering the CCF shape. Santerne et al. (2015) showed that under some configurations, such an unresolved double-lined spectroscopic binary (hereafter SB2) would produce only a very weak correlation between the RV and the BIS, making it looking like a planetary signal. However, these authors also emphasized that even in such configurations, the FWHM should still be correlated to the RV, while here the $\theta$ Cyg RV-FWHM correlation is weak. In contrast, a more convincing example of a RV-FWHM correlation probably induced by a spectroscopic binary can be found for HD 191195 (Sect. 3.3.11). For masses between $\sim 0$ (i.e. negligible compared to the primary) and $0.5 M_{\odot}$, such a companion on a (nearly circular) 150-day orbital period would have a sma between 0.6 and 0.7 au, respectively. This falls into the separation range covered by the interferometric detection limits $\left(\leq 0.5 M_{\odot}\right)$ from White et al. (2013). The $H$-band contrasts provided by these authors may well translate into larger contrasts in the $V$ band (i.e. the SOPHIE range) that would exclude significant effects on the CCF and RV. While not conclusive, this is another argument against the stellar companion hypothesis.

Finally, the planetary (or multi-planetary) hypothesis was already well explored by D09, but with no satisfying results. We tried to fit various Keplerian models with yorbit to $\theta$ Cyg RV (from 1 to 4 planets, with/without trends) and considered the cases detailed by D09, but to no avail. Furthermore, we also considered the ELODIE RV data set from D09, and the RV data set published by Howard \& Fulton (2016). Both these data sets exhibit RV variations of $\sim 300 \mathrm{~m} \mathrm{~s}^{-1}$ amplitude with a periodic character at about 150 days. We also tried to fit these different RV data sets with yorbit, either separately or by combining them. However, we did not obtain a satisfying solution. Furthermore, the main $\sim 150$-day RV periodicity seems rather variable on our timebase, going from $\sim 125$ to $\sim 180$ days if dividing our timebase in several slices (Appendix D).

In conclusion, we consider that the origin of $\theta$ Cyg mid-term $\mathrm{RV}$ variations cannot be definitely determined. The variability of the RV variation period, the similarity of the RV, BIS and FWHM periodograms and the flat (RV, BIS) diagram make it a truly puzzling case.

\subsection{RV long-term trends and stellar binaries}

In this section, we describe fourteen massive and/or distant companions that we confidently identified in our survey. Given our data, most of them must be spectroscopic binaries (SB), while the others are candidate SB. We proceeded in the same way as done and explained before in Paper IX:

1. we identified the companion presence based on various diagnosis - RV variations, flat (RV, BIS) diagram, CCF variability, RV-FWHM correlation;

2. we distinguished between double-lined (SB2) and singlelined (SB1) binaries based on the presence or absence, respectively, of CCF distortions and/or RV-FWHM correlation;

3. if possible, we fitted the RV with various models (linear, quadratic, or Keplerian) using both our SOPHIE data and, whenever possible, other RV data sets available in the literature. We then explored the RV residuals looking for lower-mass companions;

4. for our targets with long-term (linear or quadratic) RV trends, we constrained the companion properties $\left(m_{\mathrm{p}} \sin i\right.$, sma) given the available data, as we did in Paper IX. Briefly, we assumed the companion period to be at least equal to the observation time baseline, and the RV amplitude induced by the companion to be at least equal to the span of the observed RV trend, considering a circular orbit. We then deduced the corresponding minimal mass versus sma relation (see Paper IX for details). If appropriate, we looked for additional constraints from the literature.

\subsubsection{HD 10453}

HD 10453 (HIP 7916, F5V) shows high-amplitude ( $2.2 \mathrm{~km} \mathrm{~s}^{-1}$ peak-to-peak) RV variations over our 1590-day time baseline, along with a distorted CCF and high-amplitude 

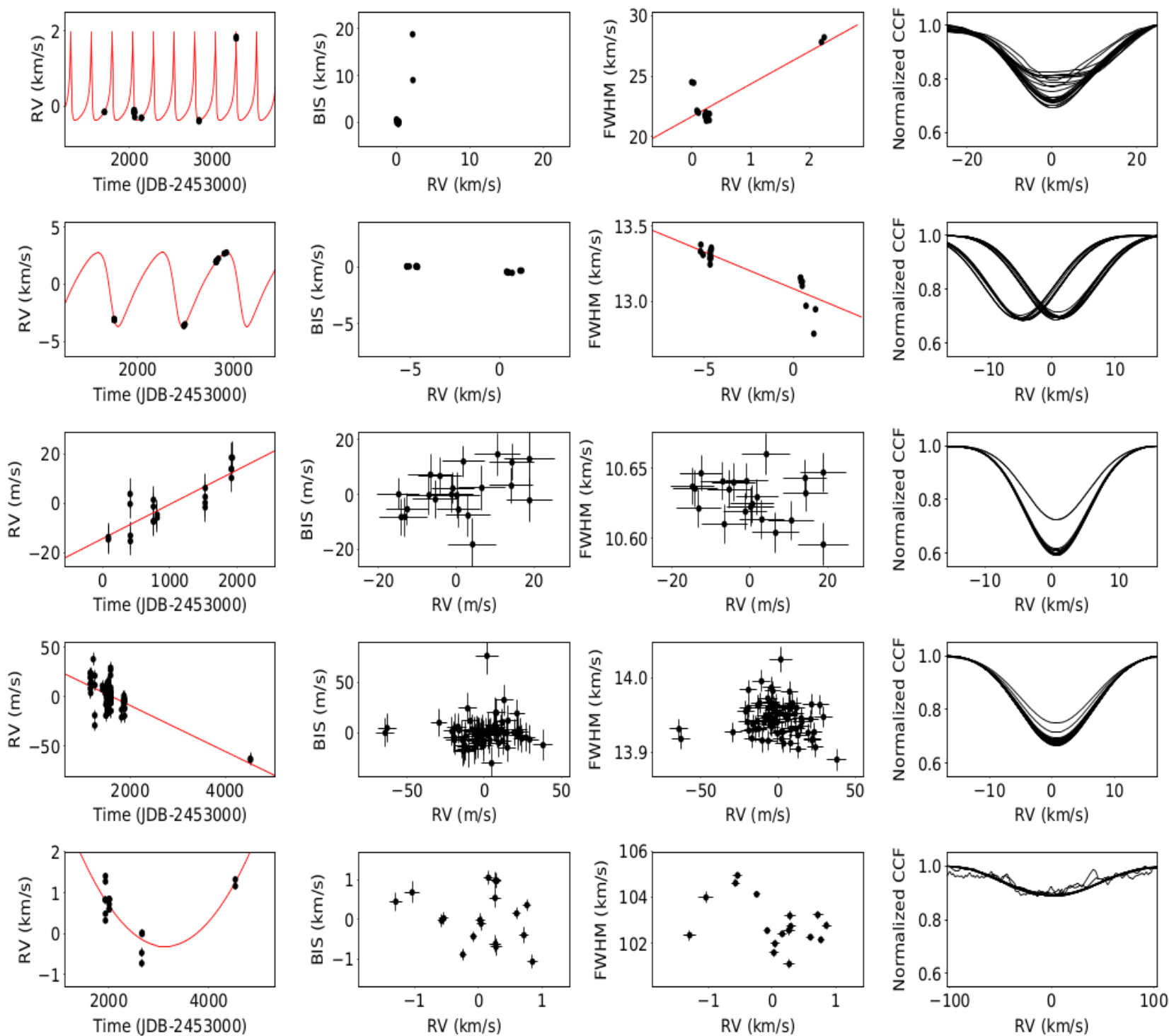

Fig. 5. Candidate binary companions (first part). From top to bottom rows: HD 10453, HD 20395, HD 43318, HD 82328, HD 143584. From left to right columns: RV time series, BIS vs RV, FWHM vs RV, stacked CCF. The best yorbit fit of the RV variations is overplotted in red to the RV. On the RV-FWHM diagram, the RV-FWHM correlation is overplotted in red if the absolute Pearson correlation coefficient is $\geq 0.6$.

BIS and FWHM variations (Fig. 5) that are characteristic of a SB2 binary. We tried to fit the RV variations with yorbit using a Keplerian model. When letting free all the orbital parameters, the best model corresponds to a companion with $m_{\mathrm{p}} \sin i=31.1 M_{\mathrm{Jup}}, P=249.2 \pm 20.5$ days and $e \simeq 0.76$. However, given the obvious SB2 nature of this companion considering the line profile variations, and that our temporal sampling far from covers the orbital phase of the binary secondary component, we consider that the actual companion mass has to be much larger than this estimation. We note that if running yorbit with various forced periods in the range of 100-3000 days, the solutions we find are almost as good as the free-parameter solution in terms of $\chi^{2}$, highlighting our incomplete temporal sampling.

HD 10453 is a known binary. A stellar companion was resolved by speckle interferometry at a projected separation of $\sim 0.05 "$ ( 2 au, Hartkopf et al. 2012) while Riddle et al. (2015) imaged a stellar companion at a projected separation of $\sim 0.18$ " $(\sim 7 \mathrm{au})$. Given the uncertainties on our orbital parameters, we cannot conclude whether the RV variations are induced by the companion detected by Hartkopf et al. (2012) or Riddle et al. (2015), or whether the HD 10453 system has more components.

\subsubsection{HD 16232}

As presented in Sect. 3.1.2, we detect a long-term linear trend in our SOPHIE RV, along with a composite (RV, BIS) diagram indicative of a companion-induced trend (Fig. D.2). A linear RV trend was already reported by Kane et al. (2015). To derive the best constraints on this companion, we combined our SOPHIE RV data set with the RV from K15 and fitted a linear RV drift with yorbit. Such a combination of different RV data sets is possible as yorbit includes the RV offsets between the data sets as fitting parameters. The best solution is a linear trend with a $-77.4 \pm 3.2 \mathrm{~m} \mathrm{~s}^{-1} \mathrm{yr}^{-1}$ slope and a $802 \mathrm{~m} \mathrm{~s}^{-1}$ amplitude over the combined RV data set (Fig. 6). The companion responsible for this RV trend has then to be more massive than $10 M_{\text {Jup }}$ and has to be orbiting further out than $5.2 \mathrm{au}$ (Fig. 7).

A stellar companion to 30 Ari $\mathrm{B}$ has been imaged at a projected separation of $\sim 0.54 "$ ( $\sim 22$ au, Riddle et al. 2015, $\mathrm{K} 15$ ), with a mass estimated to be roughly $0.5 M_{\odot}$ (Roberts et al. 2015). In addition, Kane et al. (2015) estimated that the mass of the imaged companion would have to be higher than $0.29 M_{\odot}\left(\sim 304 M_{\text {Jup }}\right)$ to explain the RV linear trend they detected. 

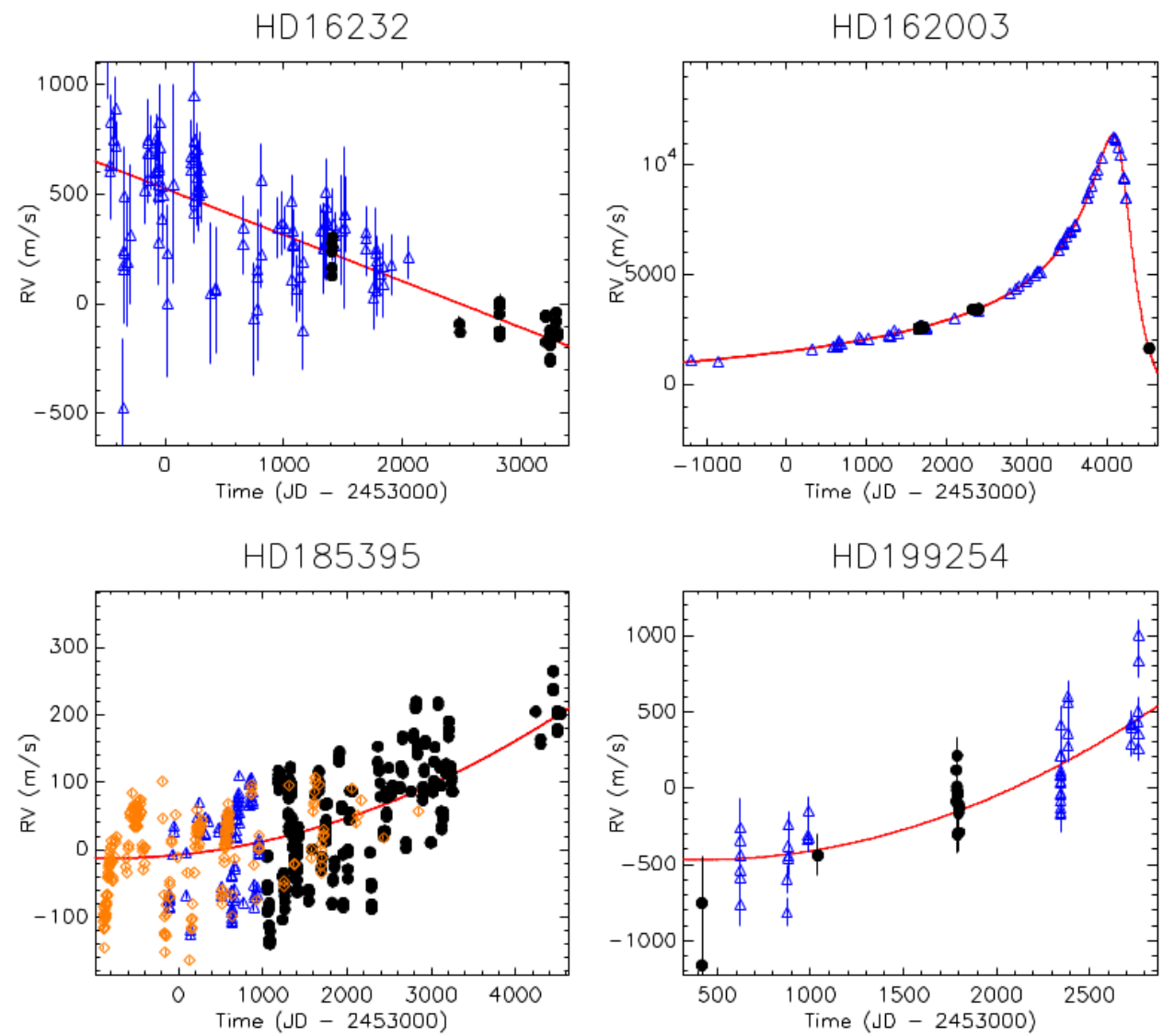

Fig. 6. Targets with combined RV data sets. In all panels, RV data from our SOPHIE survey are plotted as black dots, and the yorbit fit we derived from the combined RV data sets (either linear, quadratic, or Keplerian) is overplotted in red. Top left panel: HD 16232 - RV data from Kane et al. (2015) are plotted as blue triangles. Top right panel: HD 162003 - RV data from Endl et al. (2016) are plotted as blue triangles. Bottom left panel: HD 185395 - our ELODIE RV data (Desort et al. 2009) are plotted as blue triangles and RV data from Howard \& Fulton (2016) are plotted as orange diamonds. Bottom right panel: HD 199254 our HARPS RV data (Paper IX) are plotted as blue triangles.
The mass and separation estimated by Roberts et al. (2015) for the companion they imaged are compatible with our constraints on the minimal mass and sma of the companion responsible for the long-term RV trend detected both in our SOPHIE data and in K15 data (Fig. 7).

\subsubsection{HD 20395}

HD 20395 (14 Eri, HIP 15244, F5V) shows high-amplitude $\left(\sim 6.4 \mathrm{~km} \mathrm{~s}^{-1}\right) \mathrm{RV}$ variations along with a flat (RV, BIS) diagram, large variations in the CCF, and an apparent RV-FWHM anti-correlation (Fig. 5) that are characteristic of a spectroscopic binary. We used the yorbit software to fit the RV variations. The best solution corresponds to a low-mass stellar companion with $P=675.2 \pm 5.9$ days, $m_{\mathrm{p}} \sin i \sim 163 M_{\mathrm{Jup}}$, and $e=0.33 \pm$ 0.02 (corresponding to a $\sim 1.7$ au sma). However, given that our orbital phase coverage is not complete, we cannot exclude longer periods (and higher masses) for HD 20395B. If constraining the companion orbital period to wider ranges (up to $2.10^{4}-3.10^{4}$ days) with yorbit, reliable orbital solutions can be found (with sma up to $\sim 15 \mathrm{au}, m_{\mathrm{p}} \sin i$ up to $\sim 350 M_{\mathrm{Jup}}$, and eccentricities up to 0.8). HD 20395 has been reported as an astrometric binary based on its proper motion (Makarov \& Kaplan 2005; Frankowski et al. 2007), but without estimations of the orbital parameters.

\subsubsection{HD 43318}

HD 43318 (HIP 29716, F5V) shows a small $\left(5 \pm 0.7 \mathrm{~m} \mathrm{~s}^{-1} \mathrm{yr}^{-1}\right)$ long-term linear drift in its RV over our 1800-day timebase, along with a possibly composite (RV, BIS) diagram (Fig. 5). We thus classify this trend as a candidate companion-induced one. When corrected from the linear trend, the RV residuals show a dispersion two times smaller than the RV, and the Lomb-Scargle periodogram of the residuals show significantly less power at periods $>100$ days. Given the small amplitude of the RV trend over our timebase, the candidate distant companion can be either of GP, BD or stellar nature (Fig. 7). HD 43318 has not been reported as a binary before.

\subsubsection{HD 82328}

$\theta$ UMA (HIP 46853, F7V) shows a linear RV drift with a $78 \mathrm{~m} \mathrm{~s}^{-1}$ amplitude over our 3368-day timebase, which is best fitted with a long-term linear trend (Fig. 5). Assuming that the period of the companion responsible for the RV trend is at least equal to the total observation timebase, the companion can then be either of BD or stellar nature and has to be orbiting further than $5.3 \mathrm{au}$ from the primary (Fig. 7). HD 82328 is a known physical binary with a $\sim 5^{\prime \prime}$ projected separation (Allen et al. 2000). Recently, Tokovinin (2014) has estimated the orbital parameters of the companion based on the absolute $V$-magnitudes of the components and assuming that the projected separation corresponds to the actual sma of the companion, finding $P \sim 322 \mathrm{yr}$ and $m_{\mathrm{p}} \sin i=0.16 M_{\odot}$. Such a companion is compatible with the detected RV trend (Fig. 7).

\subsubsection{HD 91312}

HD 91312 (HIP 51658, A7IV) exhibits a large RV drift (of $\sim 4.1 \mathrm{~km} \mathrm{~s}^{-1}$ amplitude) over our 2635-day timebase (Fig. 8). As we cannot compute the line profiles for this target (too few spectral lines are available for SAFIR computation), we cannot check the (RV, BIS) diagram. Even so, we conclude that this RV drift is most probably induced by a companion because its amplitude is far larger than the short-term RV dispersion induced by 

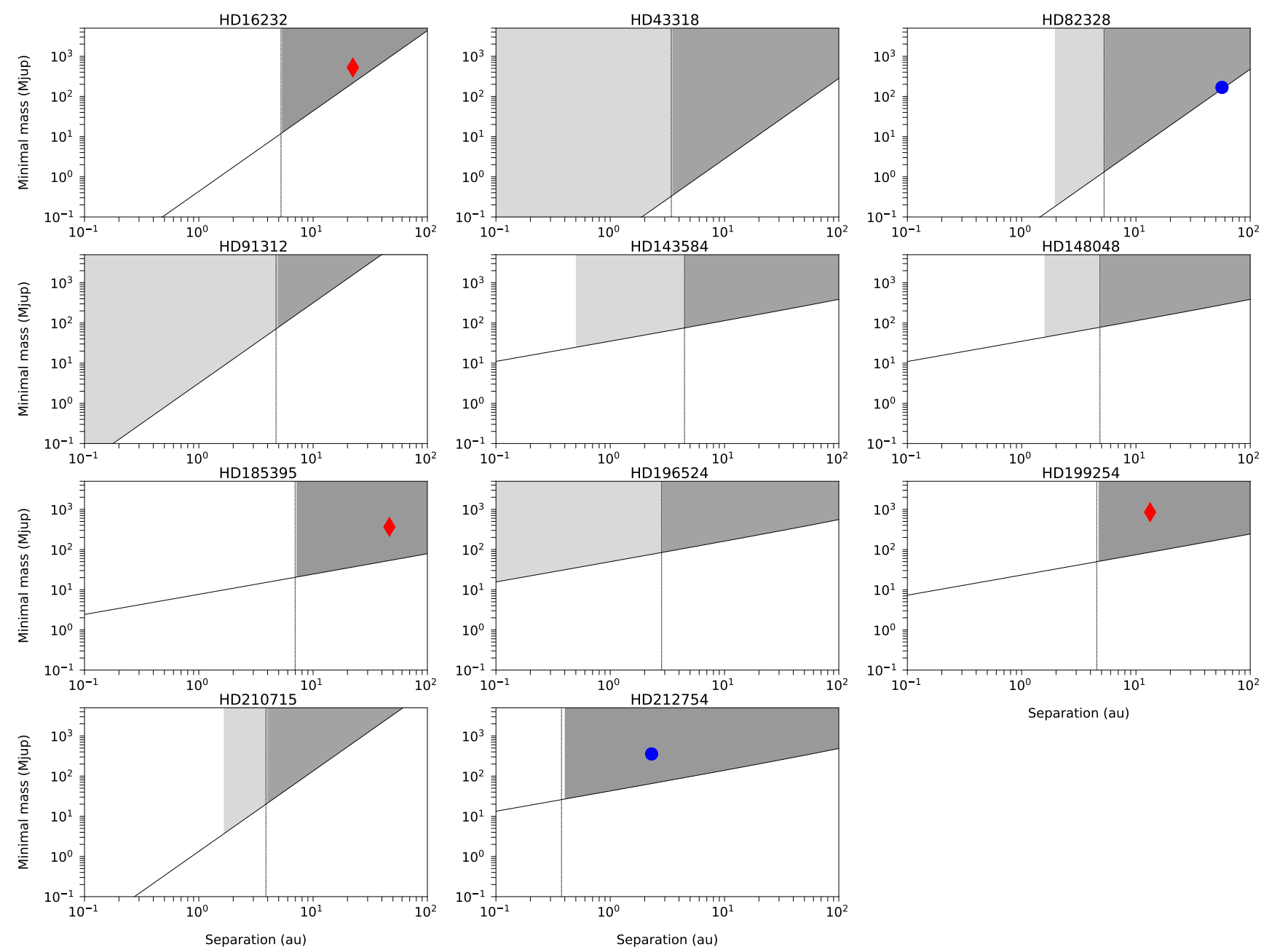

Fig. 7. Constraints on the $m_{\mathrm{p}} \sin i$ and sma of the companions to our targets with RV linear or quadratic trends. Dark grey: possible (mass, sma) domain for the companion given the available RV data, assuming a circular orbit and an orbital period at least equal to our total time baseline. Light grey: extended (mass, sma) domain in the case of orbital periods smaller than our total time baseline can still be considered, given the observation temporal sampling. Red diamonds: previously imaged companions from the literature: HD 16232 - DI from Roberts et al. (2015); HD 185395 DI from Desort et al. (2009); HD 199254 - DI from De Rosa et al. (2014). Blue dots: companions previously detected with astrometry and/or RV from the literature: HD 82328 - astrometry from Tokovinin (2014); HD 212754 - RV from Griffin (2010), and astrometry from Goldin \& Makarov (2007) and Tokovinin (2014).

stellar pulsations for this spectral type. This long-term RV drift is best fitted by a linear trend with $\mathrm{a}-566 \pm 25 \mathrm{~m} \mathrm{~s}^{-1} \mathrm{yr}^{-1}$ slope; once corrected from the drift, the RV dispersion decreases from $1.75 \mathrm{~km} \mathrm{~s}^{-1}$ to $415 \mathrm{~m} \mathrm{~s}^{-1}$.

HD 91312 is a known wide (23" or 796 au) visual binary, which is dynamically linked according to Kiyaeva et al. (2008). The very large projected separation of this companion makes it unlikely to be at the origin of the RV drift we detect. This RV trend is then likely induced by an unknown companion. Assuming an orbital period larger than our time baseline, this companion has then to be of stellar mass and has to orbit further than $4.7 \mathrm{au}$ around the primary (Fig. 7). Interestingly, HD 91312 is still young ( $200 \mathrm{Myr})$ and shows an IR excess characteristic of the presence of a cold debris disk, according to Rhee et al. (2007). A recent SED analysis (Rodriguez \& Zuckerman 2012) gives a dust radius of $179 \mathrm{au}$; this could then be a circumbinary disk.

\subsubsection{HD 143584}

HD 143584 (HIP 78296, F0IV) exhibits high-amplitude $\left(\sim 2 \mathrm{~km} \mathrm{~s}^{-1}\right) \mathrm{RV}$ variations over our 2596-day timebase on both

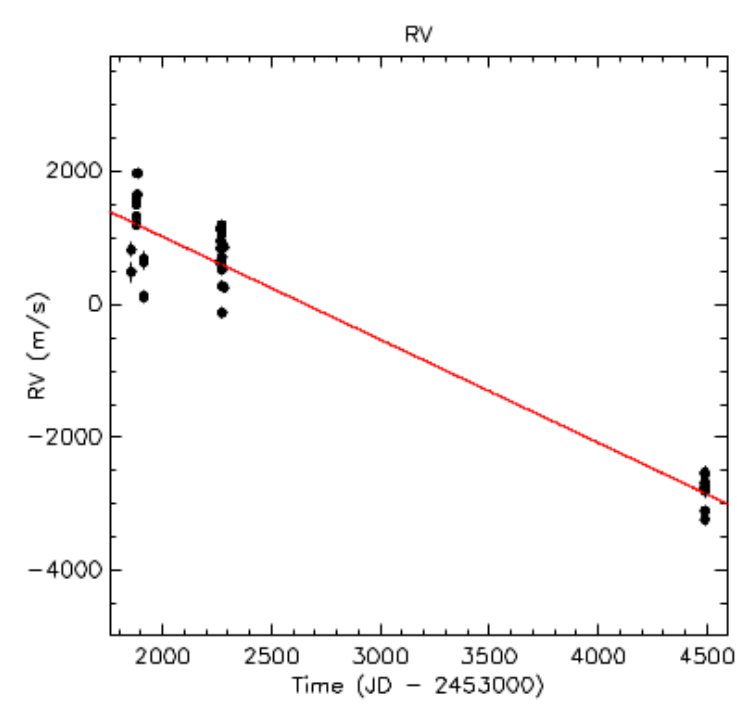

Fig. 8. HD 91312 RV time series. The assumed linear fit is overplotted in red to the RV. 
the short and long term, along with a composite (RV, BIS) diagram (Fig. 5). This is best explained by the presence of both a massive companion and stellar pulsations. The RV variations are best fitted with a long-term quadratic trend, even if periods shorter than our time baseline could still be possible. The dispersion of the RV residuals is $291 \mathrm{~m} \mathrm{~s}^{-1}$, compared to the $592 \mathrm{~m} \mathrm{~s}^{-1} \mathrm{RV}$ dispersion. Assuming an orbital period larger than our time baseline, the companion responsible for the RV longterm variations would have to be of stellar nature and would have to orbit further than 4.5 au from the primary (Fig. 7). HD 143584 has not been reported as a binary before.

\subsubsection{HD 148048}

$\eta$ Umi (HIP 79822, F5V) exhibits long-term RV variations that are best fitted by a quadratic trend of $1600 \mathrm{~m} \mathrm{~s}^{-1}$ amplitude over our 2988-day timebase. The (RV, BIS) diagram is composite with a horizontal spread induced by a companion and a vertical spread induced by stellar pulsations (Fig. 9). Assuming an orbital period larger than our time baseline, the companion responsible for these RV variations would have to be of stellar nature and would have to orbit further than 4.8 au from the primary (Fig. 7). HD 148048 has not been reported as a binary before.

\subsubsection{HD 162003}

$\psi^{1}$ Dra A (HIP 86614, F5IV-V) shows high-amplitude $\left(\sim 2 \mathrm{~km} \mathrm{~s}^{-1}\right)$, long-term RV variations over our 2890-day time baseline, along with a flat (RV, BIS) diagram (Fig. 9) characteristic of a companion. Furthermore, the RV and FWHM variations are strongly correlated (Pearson's coefficient of 0.75), which make it a slightly SB2 binary.

HD 162003 has previously been reported as a spectroscopic binary. Based on $\sim 40 \mathrm{RV}$ measurements spanning over $\sim 1550$ days, Toyota et al. (2009) detected a long-term RV trend of quadratic shape that they attributed to an unseen companion of $m_{\mathrm{p}} \sin i \sim 50 M_{\mathrm{Jup}}$, with a sma lower than 140 au (assuming a circular orbit) so that it remains on a stable orbit given the existence of the wide binary $\psi^{1}$ Dra B (HD 162004) at a projected separation of $\sim 30^{\prime \prime}(\sim 667 \mathrm{au})$ from HD 162003. Gullikson et al. (2015) detected this unseen companion (hereafter $\psi^{1}$ Dra C) by looking for a secondary peak in the CCF of HD 162003 spectra, in a way similar to Bouchy et al. (2016). From this analysis, they estimated that $\psi^{1}$ Dra $C$ had a mass of $\sim 0.7 M_{\odot}$, making it of stellar nature, and a sma of $\sim 9$ au. Finally, Endl et al. (2016) directly detected $\psi^{1}$ Dra $\mathrm{C}$ with speckle imaging on the one hand, and 85 RV measurements spanning 15 yr (2000-2015) on the other hand, estimated the orbital parameters $m_{\mathrm{p}} \sin i \sim 550 \pm 5 M_{\mathrm{Jup}}$, $e=0.67$, and $P=6650 \pm 160$ days, corresponding to a $\sim 8.7 \pm$ 0.1 au sma. We combined our SOPHIE data with the RV from Endl et al. (2016) and fitted a single Keplerian model to the combined RV data set with yorbit. Our best solution corresponds to a companion with a $661 \pm 107 M_{\text {Jup }}$ minimal mass, a sma of $24.3 \pm 3.8$ au and an eccentricity $e=0.87 \pm 0.02$ (Fig. 6). We conclude that the RV variations that we detect in our SOPHIE data are induced by $\psi^{1}$ Dra C. However, the differences between our Keplerian model and the one derived by Endl et al. (2016) show that the sampling of the companion orbit is still not complete enough to adequately cover the period, meaning that large uncertainties still remain on its orbital parameters.

\subsubsection{HD 185395}

As presented in Sect. 3.2, we detect a long-term quadratic RV drift in addition to the RV mid-term complex variability of $\theta$ Cyg. This specific RV long-term trend is induced by a distant companion, as shown by the flat (RV, BIS) diagram (Fig. D.3). To derive the best constraints on this companion, we combined our SOPHIE RV data set to the RV acquired with ELODIE before (Desort et al. 2009) and to the RV data from Howard \& Fulton (2016). This allowed us to expand the timebase to 5422 days (14.8 yr). Over the combined RV data set, the RV quadratic trend induced by the distant companion has an amplitude of $\sim 370 \mathrm{~m} \mathrm{~s}^{-1}$ (Fig. 6). The companion responsible for this trend is then either of BD or stellar nature and has to orbit further than 7 au from the primary (Fig. 7).

A distant stellar companion to $\theta$ Cyg was imaged by Desort et al. (2009) as a projected separation of 46.5 au from the primary. Based on the measured contrast between the two components and on stellar evolutionary models, these authors deduced a mass of $\sim 0.35 M_{\odot}$ for the companion. These estimated mass and projected separation are compatible with the constrains we derive from the RV long-term trend (Fig. 7).

\subsubsection{HD 191195}

HD 191195 (F5V, $1.49 M_{\odot}$ ) exhibits complex RV variations, with a RV peak-to-peak amplitude of $272 \mathrm{~m} \mathrm{~s}^{-1}$ and a dispersion of $56.5 \mathrm{~m} \mathrm{~s}^{-1}$ (Fig. D.4). We consequently followed this target intensively, acquiring 265 spectra over a 3191-day ( $\sim 8.7 \mathrm{yr})$ time baseline. The RV Lomb-Scargle shows multiple peaks between 100 and $10^{3}$ days, but they are all aliases of a single 300-day periodicity, as shown by the RV CLEAN periodogram. The BIS does not show high-amplitude variations (dispersion of $18 \mathrm{~m} \mathrm{~s}^{-1}$ ), hence the flat (RV, BIS) diagram. As did the RV, the FWHM exhibits high-amplitude $\left(266 \mathrm{~m} \mathrm{~s}^{-1}\right.$ peak-to-peak) complex variations. The FWHM Lomb-Scargle and CLEAN periodograms are strikingly similar to those of the RV, with a clear, single peak at $\sim 300$ days in the CLEAN periodogram. We emphasize that HD 191195 RV and FWHM are strongly anti-correlated, with a Pearson's correlation coefficient of -0.65 (Fig. D.4).

Given HD $191195 v \sin i$ of $5.5 \mathrm{~km} \mathrm{~s}^{-1}$, the flat (RV, BIS) diagram points towards a companion as the source of the observed RV variability. We tried to fit single- or multi-companion Keplerian models to HD $191195 \mathrm{RV}$, including one to three planets and an additional linear or quadratic trend. However, none of them was able to reproduce convincingly the RV variations. We consider that this complex RV variability is induced by a faint stellar companion rather than by a planetary companion. This is strongly supported by the observed RV-FWHM anti-correlation, which can be explained by an unresolved SB2 binary (see Santerne et al. 2015, and the discussion about $\theta$ Cyg in Sect. 3.2). Given the relatively small $v \sin i$ of HD 191195, we might be seeing this possible binary system under an inclined or even close to pole-on configuration, which would explain such a low RV amplitude for a companion of stellar nature. HD 191195 has not been reported as a binary before.

\subsubsection{HD 196524}

$\beta$ Del (HIP 101769, F5IV) shows high-amplitude $\left(2 \mathrm{~km} \mathrm{~s}^{-1}\right)$ RV variations over our 1179-day timebase, along with a flat (RV, BIS) diagram characteristic of a companion (Fig. 9). The $\mathrm{RV}$ are best fitted by a quadratic model. Assuming an orbital period larger than our time baseline, the companion responsible for this RV trend would have to be of stellar mass and would have to orbit further than 2.8 au from the primary (Fig. 7). 

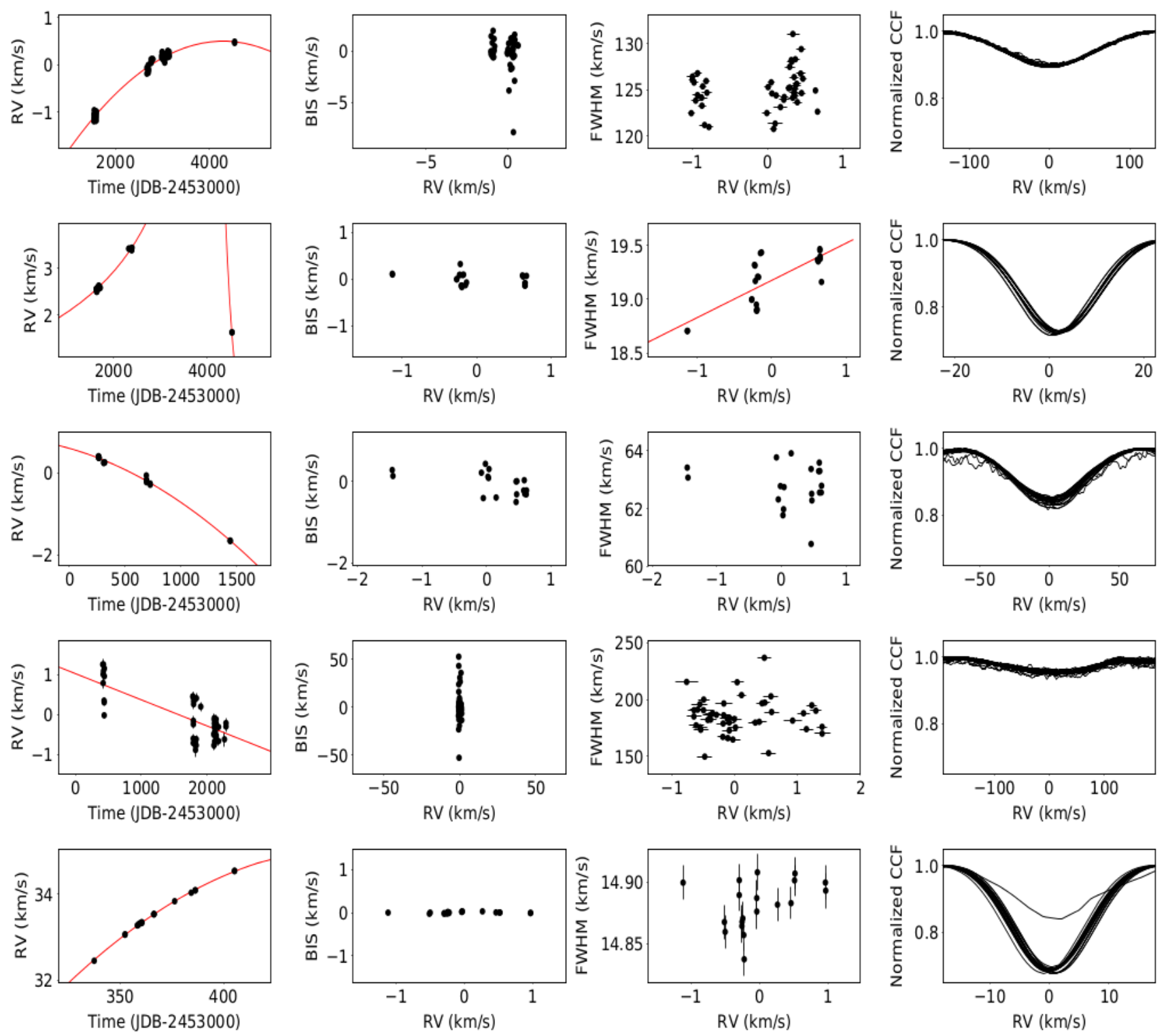

Fig. 9. Candidate binary companions (second part). From top to bottom rows: HD 148048, HD 162003, HD 196524, HD 210715, HD 212754. From left to right columns: RV time series, BIS vs RV, FWHM vs RV, stacked CCF. The best yorbit fit of the RV variations is overplotted in red to the RV. On the RV-FWHM diagram, the RV-FWHM correlation is overplotted in red if the absolute Pearson correlation coefficient is $\geq 0.6$.

HD 196524 was already known as a spectroscopic binary (Pourbaix et al. 2004). Based on astrometric data, Malkov et al. (2012) estimated its period to be of $\sim 26.7 \mathrm{yr}$, its eccentricity of 0.36 , and its sma of $\sim 0.44^{\prime \prime}(\sim 13.6 \mathrm{au})$. At such a separation and given the mass constraints we bring, the companion would have a mass greater than $\sim 200 M_{\text {Jup }}$ (Fig. 7).

\subsubsection{HD 199254}

We have already detected HD 199254 (HIP 103298, A5V) as a spectroscopic binary thanks to our HARPS RV (see Paper IX). We have also observed this target with SOPHIE and we detected a long-term, high-amplitude RV drift. When combining the SOPHIE and HARPS RV data and fitting a trend model with yorbit, we found the best solution to be a slightly quadratic trend of $923 \mathrm{~m} \mathrm{~s}^{-1}$ amplitude over the total time baseline of 2400 days (Fig. 6). Given our combined RV data, the companion responsible for such a RV trend is of stellar nature and orbits further than 4.5 au from the primary (Fig. 7). A stellar companion with properties compatible with these constraints was imaged around HD 199254 by De Rosa et al. (2014), see details in Paper IX.

\subsubsection{HD 210715}

HD 210715 (HIP 109521, A5V) exhibits a clear RV linear drift of $1.2 \mathrm{~km} \mathrm{~s}^{-1}$ amplitude over the 1880-day timebase. Even if the (RV, BIS) diagram is dominated by a vertical spread induced by stellar pulsations characteristic of its stellar type (Fig. 9), we consider that the RV long-term trend is induced by a distant massive companion.

HD 210715 has been reported as an astrometric binary (Makarov \& Kaplan 2005; Frankowski et al. 2007), but no constraints on the companion parameters are available in the literature. Assuming an orbital period larger than our time baseline, the unseen companion responsible for the RV long-term trend would have to be either of $\mathrm{BD}$ or stellar nature and would have to orbit further than 3.9 au from the primary (Fig. 7). 

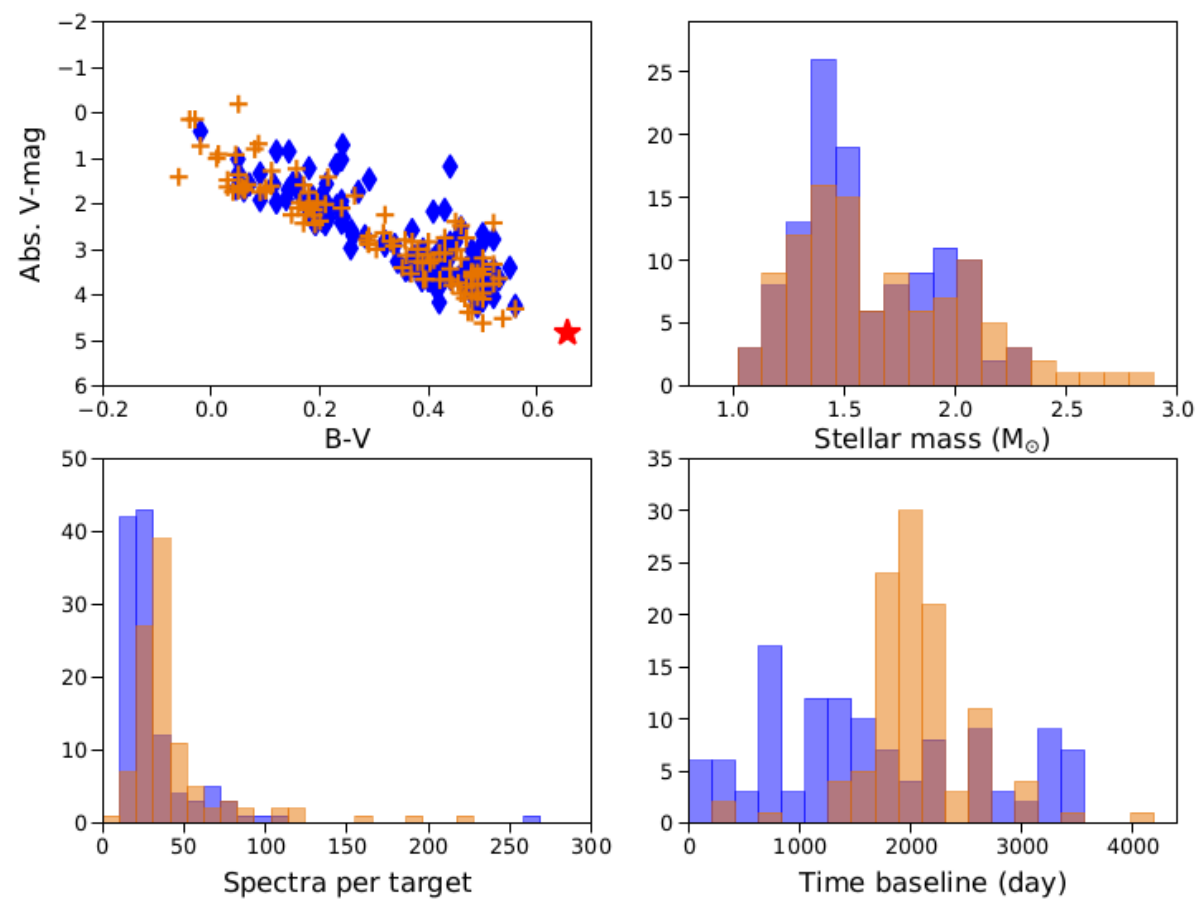

Fig. 10. Properties of our combined sample (SOPHIE +HARPS). Top left panel: position of our targets in an H-R diagram. Blue diamonds: SOPHIE targets; orange crosses: HARPS targets. The Sun is displayed (red star) for comparison. Top right panel: stellar mass histogram of our combined sample. Blue: SOPHIE targets; orange: HARPS targets. Bottom left panel: spectrum number per target. Bottom right panel: timebase histogram.

\subsubsection{HD 212754}

We acquired only 19 RV measurements over 68 days on HD 212754 (HIP 110785, F7V), but we are able to detect a highamplitude $\left(2 \mathrm{~km} \mathrm{~s}^{-1}\right) \mathrm{RV}$ drift of quadratic shape, along with a flat (RV, BIS) diagram and large CCF variations that are indicative of a spectroscopic binary (Fig. 9). Given our small timebase, we were not able to fully determine the orbital parameters of the companion. This companion has to orbit further than 0.4 au from the primary and is most probably of stellar nature (Fig. 7), given the CCF variations.

HD 212754 is a known SB1 (Griffin 2010) and astrometric binary (Goldin \& Makarov 2007) with an orbital period of 931 days, an eccentricity of $0.3-0.4$ and a secondary minimal mass of $0.34 M_{\odot}$ (Tokovinin 2014) at a separation of $\sim 59$ mas ( 2.3 au). This already known stellar companion is most likely at the origin of the detected SOPHIE RV variations.

\section{Combined analysis of the SOPHIE + HARPS surveys}

Here, we combine our SOPHIE AF survey with the similar HARPS survey described in Paper IX to make a global analysis in terms of achieved companion detections, sensitivity and statistics in the 1- to 1000-day period range. In addition to the two GPs detected in this period range in this SOPHIE survey (HD 113337b and HD 16232b), we include the three confirmed GPs detected in our HARPS survey around two F6V targets (HD 60532b, c and HD 111998b) in the following analysis.

\subsection{Characteristics of the combined survey}

We combine the 125 AF dwarf stars of our SOPHIE sample to the 109 targets of our HARPS survey (Paper IX). We note that nine of our SOPHIE targets were also part of our HARPS sample: HD 13555, HD 25490, HD 29488, HD 102647, HD 197890 , HD 199254, HD 211976, HD 218396, and HD 222368. Our combined AF sample is thus made of $N=225$ distinct targets. In the case of the targets with both HARPS and SOPHIE observations, we selected the instrument for which we had the most RV data and the longest time span (i.e. we used the SOPHIE data for HD 102647 and HD 218396, and the HARPS data for the seven other targets) and use the corresponding data in the following.

We do not combine HARPS and SOPHIE RV data for these targets as it is not possible here to compute an accurate value of the zero-point between the two RV data sets, the SAFIR RV being relative RV. Furthermore, our detection limits are computed based on an analysis of the RV periodogram (Sect. 4.3). In this context, a HARPS + SOPHIE RV combination might lead to a biased combined periodogram and thus biased detection limits. Hence, using only the RV data set with the most data and the longest time span ensures an unbiased periodogram, but it will lead to slightly more conservative detection limits. Our analysis of the combined survey is then based on 107 targets observed with HARPS and 118 targets observed with SOPHIE. The combination of these two samples is possible because we use the same target selection process (Appendix B). Both samples are thus very similar in terms of stellar physical properties (Fig. 10).

The median time baseline of our SOPHIE survey is 1448 days (mean time baseline 1640 days), with a median spectrum number per target of 23 (average 36) acquired during a median number of 11 visits (average 17). These values are lower than those obtained for our HARPS sample (see Paper IX and Fig. 10). This difference is mostly explained by the increased performance and better observing conditions of HARPS compared to SOPHIE. When combining the two surveys, we obtain a median timebase of 1888 days (average 1832) and a median number of 30 spectra acquired per target.

\subsection{Stellar intrinsic variability}

To characterize the stellar intrinsic variability of our targets, we display in Fig. 11 the RV dispersion (after having removed the companion-induced RV variations) and the mean RV uncertainties (accounting only for the photon noise) of our targets versus their main physical properties $\left(B-V, v \sin i, M_{\star}\right)$, as we did in Paper IX. Our results are similar to those in Paper IX: the 

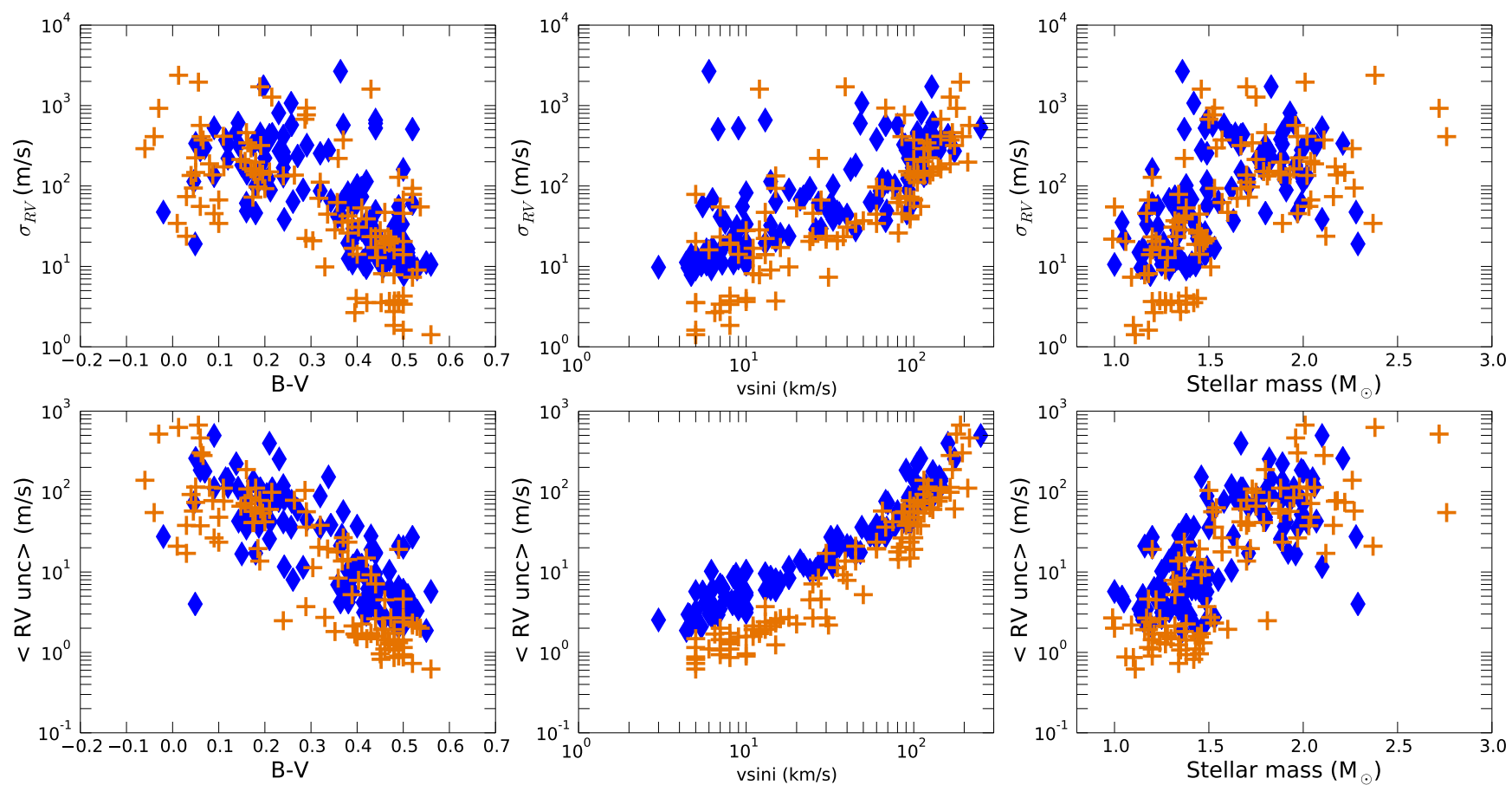

Fig. 11. Stellar intrinsic RV variability vs stellar properties. First row: stellar intrinsic RV jitter vs $B-V$, vs $v \sin i$, and vs mass (from left to right). Blue diamonds: SOPHIE targets; orange crosses: HARPS targets. Second row: averaged RV uncertainty (accounting for the photon noise only) vs $B-V$, vs $v \sin i$, and vs mass.
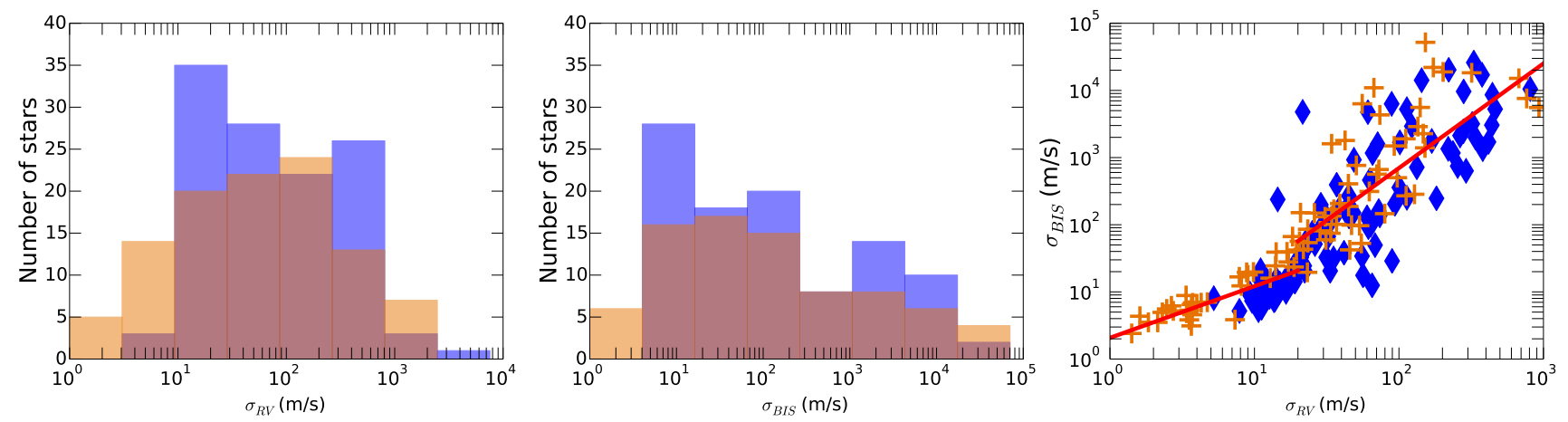

Fig. 12. Stellar intrinsic variability in our observables. Left panel: histogram of the stellar intrinsic RV jitter (blue: SOPHIE targets; orange: HARPS targets). Middle panel: histogram of the BIS dispersion. Right panel: BIS dispersion vs intrinsic RV jitter (SOPHIE targets are shown as blue diamonds and HARPS targets as orange crosses). The best power-law fits to the BIS rms vs RV rms distribution in the two variability regimes are shown as red solid lines.

$\mathrm{RV}$ uncertainties, and to a lesser degree the RV dispersion, are correlated with the $v \sin i$ and anti-correlated with the $B-V$, and consequently are loosely correlated with the stellar mass. In terms of RV and BIS dispersion, the SOPHIE and HARPS targets show the same behaviour (Fig. 12). The only remarkable differences between the two surveys are the larger mean SOPHIE RV uncertainty compared to HARPS (Fig. 11) and the relative lack of small RV and BIS dispersion values $\left(<10 \mathrm{~m} \mathrm{~s}^{-1}\right)$ for the SOPHIE survey compared to HARPS (Fig. 12). This is not induced by a difference between the two stellar samples, but rather by the decreased spectral resolution (and thus decreased RV accuracy) of SOPHIE with respect to HARPS.

The median RV dispersion is $61 \mathrm{~m} \mathrm{~s}^{-1}$ for the combined sample (191 $\mathrm{m} \mathrm{s}^{-1}$ on average), and the median RV uncertainty (not accounting for the instrumental error) is $14 \mathrm{~m} \mathrm{~s}^{-1}\left(49 \mathrm{~m} \mathrm{~s}^{-1}\right.$ on average). As we detailed in Lagrange et al. (2009) and Paper IX, the stellar jitter of AF-type MS dwarfs is mainly induced by stellar magnetic activity (spots and faculae) for our later-type (F) targets, and by high-frequency pulsations for our earlier-type (A) targets. These two variability regimes are easily seen on a BIS dispersion versus RV dispersion plot (Fig. 12). For our targets with a BIS dispersion below $\sim 20 \mathrm{~m} \mathrm{~s}^{-1}$, there is a correlation of 0.7 (Pearson) between the logarithms of the RV and BIS dispersions, and the BIS dispersion increases with the RV dispersion as

$\sigma_{\mathrm{BIS}}=2.1\left(\sigma_{\mathrm{RV}}\right)^{0.78 \pm 0.10}$

In contrast, for our targets with a BIS dispersion above $\sim 20 \mathrm{~m} \mathrm{~s}^{-1}$ (mostly pulsation-dominated jitter), the correlation is 0.73 and the BIS dispersion increases with the RV dispersion as

$\sigma_{\mathrm{BIS}}=0.5\left(\sigma_{\mathrm{RV}}\right)^{1.56 \pm 0.14}$

where the exponent to $\sigma_{\mathrm{RV}}$ happens to be twice that in Eq. (1). For a few of our SOPHIE targets with a RV jitter dominated by 

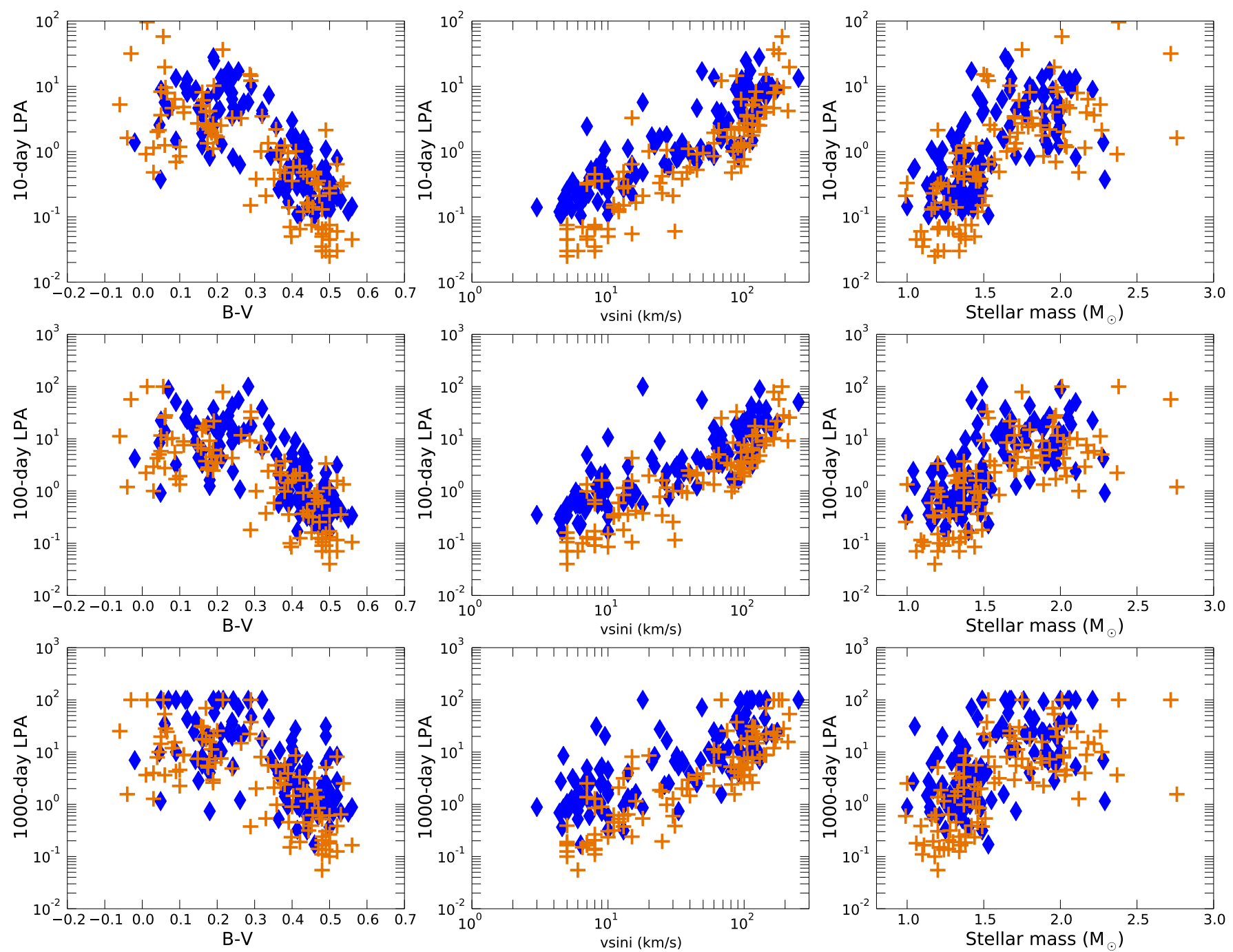

Fig. 13. Detection limits vs stellar properties. From top to bottom rows: 10 -day, $10^{2}$-day, and $10^{3}$-day LPA detection limits; vs (from left to right columns) $B-V, v \sin i$, and mass. SOPHIE targets are shown as blue diamonds and HARPS targets as orange crosses.

stellar activity, we partially corrected the RV stellar variability by using the RV-BIS anti-correlation, as we did in Paper IX. We used the same criterion: we made a linear fit of the BIS versus $\mathrm{RV}$ data and removed it from the RV if the corresponding Pearson's coefficient was above 0.7 in absolute. This was the case for six of our SOPHIE targets (Appendix A). On average, this RVBIS correction decreases the RV variation amplitude by a factor of 1.6 and the RV dispersion (rms) by a factor of 1.7 , similarly to the value we obtained in Paper IX.

\subsection{Detection limits}

We compute the minimal mass $\left(m_{\mathrm{p}} \sin i\right)$ versus period $(P)$ detection limits for each target with no confirmed GPs in our combined survey, except for two targets with too few data points $(<10)$, for which our periodogram-based detection limits are not effective, HD 196724 and HD 216627 from the HARPS sample (see Paper IX), i.e. 219 targets in total. We use the local power analysis (LPA) method (Meunier et al. 2012) to compute the detection limits in the 1 - to $2 \times 10^{3}$-day range on a log-spaced grid of 210 periods. Briefly, the LPA method compares the maximum power of the Lomb-Scargle periodogram of a synthetic planet on a circular orbit to the maximum power of the periodogram of the observed RV within a localized period range to compute the $m_{\mathrm{p}} \sin i$ detection limits at each period. Our method as well as the LPA process are fully described in Paper IX.

We display our detection limits at meaningful ranges $\left(10,10^{2}\right.$, and $10^{3}$ days) versus the main stellar properties $(B-V$, $v \sin i$, mass) in Fig. 13. On the whole, our results are the same as in Paper IX, i.e. the detection limits are correlated with the $v \sin i$ and anti-correlated with the $B-V$, in agreement with our results on the intrinsic variability. Overall, the SOPHIE targets show slightly higher detection limits than the HARPS targets (Fig. 14), which is again in agreement with the slightly decreased RV accuracy of SOPHIE compared to HARPS.

We compute the search completeness $C\left(P, m_{\mathrm{p}} \sin i\right)$ of our combined survey by combining the detection limits of all our targets, as we did in Paper IX for the HARPS targets only. For a given couple $\left(P, m_{\mathrm{p}} \sin i\right), C$ gives the fraction of stars in the combined sample for which a companion with a minimal mass $m_{\mathrm{p}} \sin i$ and an orbital period $P$ (on a circular orbit) would be detected if present, given our observations. We refer to Paper IX for details. We display our combined survey completeness in Fig. 15, along with the confirmed GPs detected in the combined survey. 

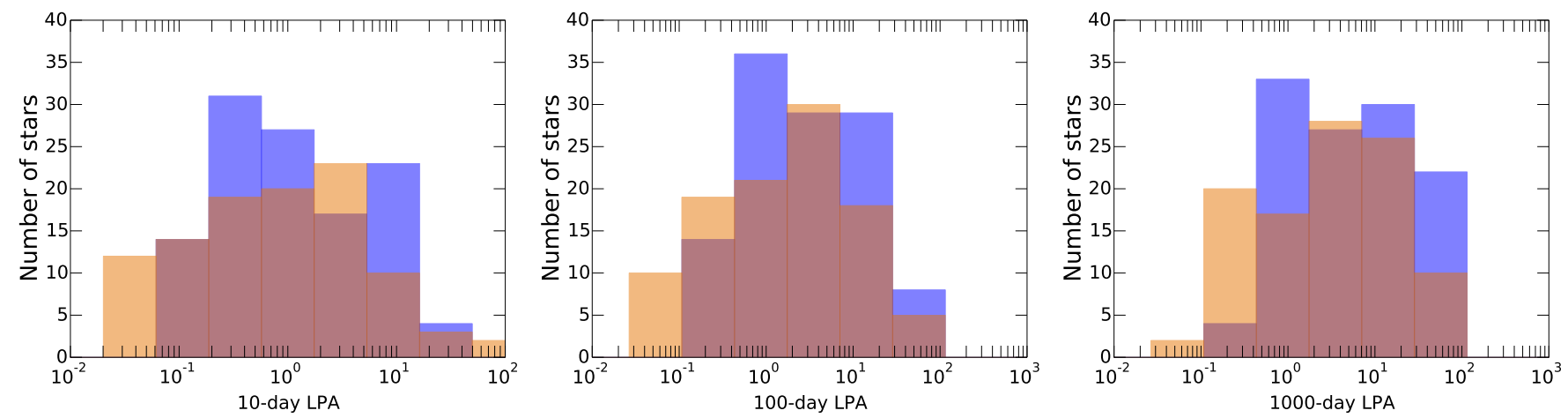

Fig. 14. Distribution of the achieved detection limits. From left to right panels: histograms of the 10-day, $10^{2}$-day, and $10^{3}$-day LPA detection limits . Blue: SOPHIE targets; orange: HARPS targets.

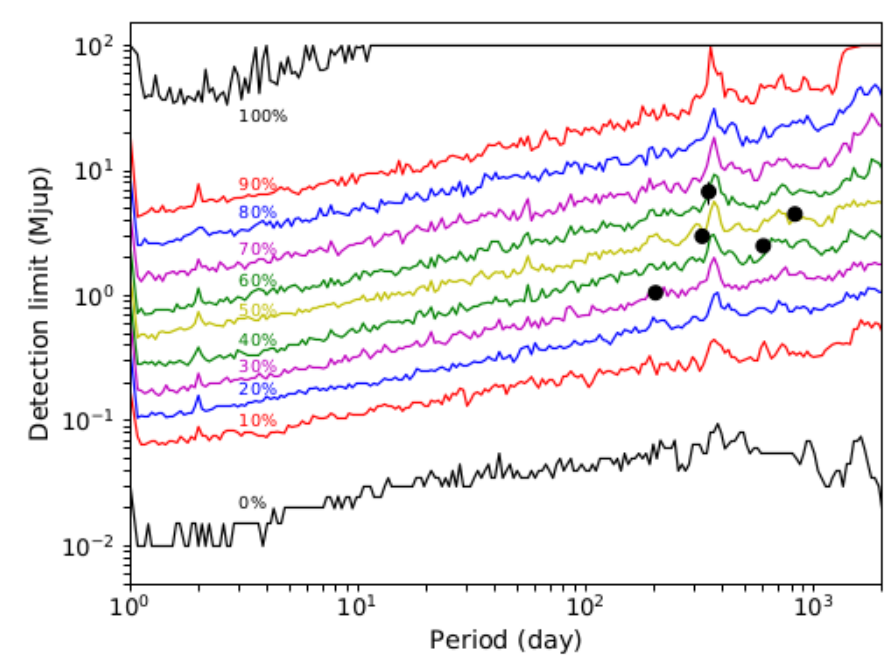

Fig. 15. Search completeness of our combined survey. From bottom to top: 0 to $100 \%$ search completeness (i.e. the fraction of stars with good enough detection limits to rule out a companion of a given $m_{\mathrm{p}} \sin i$ at a given orbital period $P$ ). Black dots: confirmed GPs detected in the combined survey. HD 16232b, whose detection was confirmed by Kane et al. (2015), is included here (see Sect. 3.1.2).

\subsection{Companion occurrence rates}

\subsubsection{Method summary}

We compute the companion frequency around AF MS stars based on the detected GPs and on the detection limits of our 225target combined sample. Our method is fully detailed in Paper IX and in Appendix C. Briefly:

1. We consider different $m_{\mathrm{p}} \sin i$ ranges $\left(0.3-1 M_{\mathrm{Jup}}\right.$ for Saturn-mass GPs, 1-13 $M_{\text {Jup }}$ for Jupiter-mass GPs, 13-80 $M_{\text {Jup }}$ for BDs) and different period ranges $\left(1-10,10-10^{2}\right.$ and $10^{2}-10^{3}$ days) to define the $\left(m_{\mathrm{p}} \sin i, P\right)$ domains $(D)$ where the companion frequency is computed.

2. We consider two target subsamples depending on the stellar mass: 104 targets with masses above $1.5 M_{\odot}$, and 121 targets with masses $\leq 1.5 M_{\odot}$.

3. We sum the search completeness $C\left(P, m_{\mathrm{p}} \sin i\right)$ over $m_{\mathrm{p}} \sin i$ and over $P$ in each defined domain $D$ to get the search completeness function $C_{\mathrm{D}}$ over $D$. Excluding the four targets with detected GPs (HD 16232, HD 113337 from the SOPHIE survey; HD 60532 and HD 111998 from the HARPS survey) and two targets with too few spectra from the HARPS survey (see above), we compute the search completeness for each domain over 219 targets (respectively 102 and 117 targets for the high and low stellar mass subsamples).

4. For each $\left(P, m_{\mathrm{p}} \sin i\right)$ domain, we estimate the number of systems with missed companions $n_{\text {miss }}$ that we potentially did not detect in our survey, based on the search completeness $C_{\mathrm{D}}$ and the number $n_{\mathrm{det}}$ of systems with at least one detected GP.

5. We derive the companion occurrence rate in each domain and for our different mass subsamples using binomial statistics, i.e. by computing the probability distribution function (PDF) of drawing $n_{\text {det }}$ systems with detected GPs among $N=225$ stars, and then computing the product of this PDF by $\left(n_{\mathrm{det}}+n_{\mathrm{miss}}\right) / n_{\mathrm{det}}$ to correct for our search incompleteness (see details in Paper IX). The companion occurrence rate corresponds to the companion frequency value with the maximum probability (i.e. to the mode of the PDF). If $n_{\mathrm{det}}=0$, we consider $n_{\mathrm{det}}^{\prime}=1$ (as an upper limit) and compute the corresponding upper limit on the occurrence rate.

\subsubsection{Results from the combined SOPHIE + HARPS survey}

We display our results in detail in Table 3 and in Fig. 16.

\section{Companion frequency versus $m_{\mathrm{p}} \sin i$.}

1. We first consider companions with masses in the 13-80 $M_{\text {Jup }}$ range. We do not detect any companions in the $1-10^{3}$ day range. Our completeness in this $m_{\mathrm{p}} \sin i$ domain is close to $100 \%$. We thus obtain an upper limit on the BD occurrence rate of $\$ 3.5-4 \%$ (at $1 \sigma$ uncertainty, see below) for both our A- and F-type samples for periods below 1000 days. The differences in occurrence rates between our stellar mass subsamples or between the different considered period ranges are negligible (Fig. 16). Given that these estimations are still upper limits on the occurrence rates (no actual detection), they are compatible with our previous statistical analysis on HARPS targets only (Paper IX). These upper limits on the $\mathrm{BD}$-mass companion occurrence rates are roughly twice as small as in Paper IX (which is in agreement with a sample two times larger).

Our upper limits on the $\mathrm{BD}$ occurrence rate around AF stars $(\leq 4 \%$ at $1 \sigma)$ are compatible with the results obtained for solarmass stars. The close BD occurrence rate for solar-mass stars is estimated to be $<1 \%$ for $P<5 \mathrm{yr}$ (or sma $\lesssim 3 \mathrm{au}$; see Grether \& Lineweaver 2006) and around $0.6 \%$ for $P \lesssim 1 \mathrm{yr}$ (Sahlmann et al. 2011; Grieves et al. 2017). Jones et al. (2017) have found an occurrence rate of $1.2_{-0.4}^{1.5} \%$ for BD companions within 5 au around evolved intermediate-mass giant stars off the main-sequence $\left(1.6_{-0.5}^{2} \%\right.$ when restricted to stars $\left.\geq 1.5 M_{\odot}\right)$, 
Table 3. GP occurrence rate around AF dwarf stars.

\begin{tabular}{|c|c|c|c|c|c|c|c|c|}
\hline \multirow{2}{*}{$\begin{array}{l}m_{\mathrm{p}} \sin i \\
\text { interval } \\
\left(M_{\mathrm{Jup}}\right)\end{array}$} & \multirow{2}{*}{$\begin{array}{l}\text { Orbital period } \\
\text { interval } \\
\text { (day) }\end{array}$} & \multirow{2}{*}{$\begin{array}{c}\text { Search } \\
\text { completeness } \\
C(\%)\end{array}$} & \multirow{2}{*}{$\begin{array}{c}\text { Detected } \\
\text { GP systems } \\
n_{\text {det }} \\
\end{array}$} & \multirow{2}{*}{$\begin{array}{c}\text { Missed } \\
\text { GP systems } \\
n_{\text {miss }} \\
\end{array}$} & \multirow{2}{*}{$\begin{array}{c}\text { GP occ. rate } \\
\text { (computed value) } \\
(\%)\end{array}$} & \multicolumn{2}{|c|}{ Confidence intervals } & \multirow{2}{*}{$\begin{array}{l}\text { GP occ. rate } \\
(\%)\end{array}$} \\
\hline & & & & & & $\begin{array}{l}1 \sigma \\
(\%)\end{array}$ & $\begin{array}{l}2 \sigma \\
(\%)\end{array}$ & \\
\hline \multirow{15}{*}{$\begin{array}{l}13-80 \\
\text { (BD) }\end{array}$} & $1-10$ & 99 & 0 & $\sim 0$ & 0.5 & $0-1.5$ & $0-2.5$ & $\leq 1.5$ \\
\hline & & 98 & $\mathbf{0}$ & $\sim 0$ & 1 & $0-3.2$ & $0-5.5$ & $\leq 3.2$ \\
\hline & & $\sim 99$ & 0 & $\sim 0$ & 0.8 & $0-2.7$ & $0-4.6$ & $\leq 2.7$ \\
\hline & $10-100$ & 95 & 0 & $\sim 0$ & 0.5 & $0-1.5$ & $0-2.6$ & $\leq 1.5$ \\
\hline & & 92 & $\mathbf{0}$ & 0.1 & 1.1 & $0-3.4$ & $0-5.9$ & $\leq 3.4$ \\
\hline & & 98 & 0 & $\sim 0$ & 0.8 & $0-2.7$ & $0-4.7$ & $\leq 2.7$ \\
\hline & $100-1000$ & 87 & 0 & 0.2 & 0.5 & $0-1.7$ & $0-2.9$ & $\leq 1.7$ \\
\hline & & 78 & $\mathbf{0}$ & 0.3 & 1.3 & $0-4.1$ & $0-7$ & $\leq 4.1$ \\
\hline & & 95 & 0 & 0.1 & 0.9 & $0-2.8$ & $0-4.8$ & $\leq 2.8$ \\
\hline & $1-100$ & 97 & 0 & $\sim 0$ & 0.5 & $0-1.5$ & $0-2.6$ & $\leq 1.5$ \\
\hline & & 95 & $\mathbf{0}$ & 0.1 & 1 & $0-3.3$ & $0-5.7$ & $\leq 3.3$ \\
\hline & & 99 & 0 & $\sim 0$ & 0.8 & $0-2.7$ & $0-4.6$ & $\leq 2.7$ \\
\hline & $1-1000$ & 94 & 0 & 0.1 & 0.5 & $0-1.6$ & $0-2.7$ & $\leq 1.6$ \\
\hline & & 89 & $\mathbf{0}$ & 0.1 & 1.1 & 0-3.6 & $0-6$ & $\leq 3.6$ \\
\hline & & 97 & 0 & $\sim 0$ & 0.9 & $0-2.8$ & $0-4.7$ & $\leq 2.8$ \\
\hline \multirow{15}{*}{$\begin{array}{l}1-13 \\
\text { (Jupiters) }\end{array}$} & $1-10$ & 84 & 0 & 0.2 & 0.5 & $0-1.8$ & $0-3$ & $\leq 1.8$ \\
\hline & & 71 & $\mathbf{0}$ & 0.4 & 1.4 & $0-4.5$ & $0-7.6$ & $\leq 4.5$ \\
\hline & & 95 & 0 & 0.1 & 0.9 & $0-2.8$ & $0-4.8$ & $\leq 2.8$ \\
\hline & $10-100$ & 72 & 0 & 0.4 & 0.6 & $0-2.1$ & $0-3.5$ & $\leq 2.1$ \\
\hline & & 49 & $\mathbf{0}$ & 1 & 2 & $0-6.5$ & 0-11 & $\leq 6.5$ \\
\hline & & 91 & 0 & 0.1 & 0.9 & $0-2.9$ & $0-5$ & $\leq 2.9$ \\
\hline & $100-1000$ & 58 & 4 & 2.9 & 3.1 & $2.2-5.5$ & $1.2-7.9$ & $3.1_{-0.9}^{+2.4}$ \\
\hline & & 30 & $\mathbf{0}$ & 2.3 & 3.2 & 0-10.4 & 0-17.7 & $\leq \mathbf{1 0 . 4}$ \\
\hline & & 82 & 4 & 0.9 & 4.1 & $2.9-7.1$ & $1.6-10.2$ & $4.1_{-1.2}^{+3}$ \\
\hline & $1-100$ & 78 & 0 & 0.3 & 0.6 & $0-1.9$ & $0-3.2$ & $\leq 1.9$ \\
\hline & & 60 & $\mathbf{0}$ & 0.7 & 1.6 & $0-5.3$ & 0-9 & $\leq 5.3$ \\
\hline & & 93 & 0 & 0.1 & 0.9 & $0-2.9$ & $0-4.9$ & $\leq 2.9$ \\
\hline & $1-1000$ & 71 & 4 & 1.6 & 2.5 & $1.8-4.5$ & $1-6.4$ & $2.5_{-0.7}^{+2}$ \\
\hline & & 50 & $\mathbf{0}$ & 1 & 2 & $0-6.3$ & 0-10.8 & $\leq 6.3$ \\
\hline & & 89 & 4 & 0.5 & 3.7 & $2.6-6.5$ & $1.5-9.3$ & $3.7_{-1.1}^{+2.8}$ \\
\hline \multirow[t]{5}{*}{$0.3-1$ (Saturns) } & $1-10$ & 76 & 0 & 0.3 & 1.1 & $0-3.5$ & $0-6$ & $\leq 3.5$ \\
\hline & $10-100$ & 56 & 0 & 0.8 & 1.5 & $0-4.8$ & $0-8.2$ & $\leq 4.8$ \\
\hline & $100-1000$ & 31 & 0 & 2.2 & 2.7 & $0-8.6$ & $0-14.7$ & $\leq 8.6$ \\
\hline & 1-100 & 66 & 0 & 0.5 & 1.3 & $0-4.1$ & $0-6.9$ & $\leq 4.1$ \\
\hline & $1-1000$ & 54 & 0 & 0.8 & 1.5 & $0-4.9$ & $0-8.4$ & $\leq 4.9$ \\
\hline
\end{tabular}

Notes. The parameters are displayed in normal, bold, or italic fonts when considering the full star sample, the most massive $\left(>1.5 M_{\odot}\right)$ stars only or the least massive $\left(\leq 1.5 M_{\odot}\right)$ stars only, respectively. For $m_{\mathrm{p}} \sin i$ in the $0.3-1 M_{\text {Jup }}$ range, we display the GP occurrence rate and other parameters only for our low- $M_{\star}$ subsample, as our completeness is almost null in this domain for our higher mass targets. Column 6 gives the value derived from the binomial statistics based on $n_{\text {det }}$ or $n_{\text {det }}^{\prime}$, while Col. 9 gives the corresponding GP occurrence rate or upper limit at a $1 \sigma$ uncertainty.

which is also compatible with our constraints. Interestingly, these authors did not detect any BD companions within 2 au of their targets, which is in agreement with our results. In short, we have not found any significant differences to date in terms of close BD companion frequency between AF dwarfs and FGK dwarfs, and between AF dwarfs and GK subgiants.

2. We then consider Jupiter-mass companions $\left(1 \leq m_{\mathrm{p}} \sin i \leq\right.$ $\left.13 M_{\text {Jup }}\right)$. The GP system occurrence rate for our full AF sample in the $1-10^{3}$ day range is $2.5_{-0.7}^{+2} \%$, which is slightly lower than (but still compatible with) the $3_{-0.6}^{+4.7} \%$ value we obtained in Paper IX. If looking at our two stellar mass subsamples separately, we find that the five planetary-mass companions with periods within $10^{3}$ days we considered in this analysis all happen to orbit around stars $<1.5 M_{\odot}$. For this low- $M_{\star}$ subsample, our survey completeness is close to $90 \%$ (almost the same as in Paper IX); the occurrence rate is $3.7_{-1.1}^{+2.8} \%\left(P \leq 10^{3}\right.$ days $)$, which is very close to the value we found in Paper IX, but with a significantly lower uncertainty. This result is compatible with the $\sim 4 \pm 1 \%$ GP frequency found by Cumming et al. (2008) for GPs above $1 M_{\text {Jup }}$ with sma below 2 au orbiting around FGK solar-like stars.

For our high-mass subsample, we do not detect any GPs and get a completeness of $50 \%$. We thus constrain the 1-13 $M_{\text {Jup }}$ GP system occurrence rate around A-type stars to be $\lesssim 6 \%(1 \sigma)$ for 

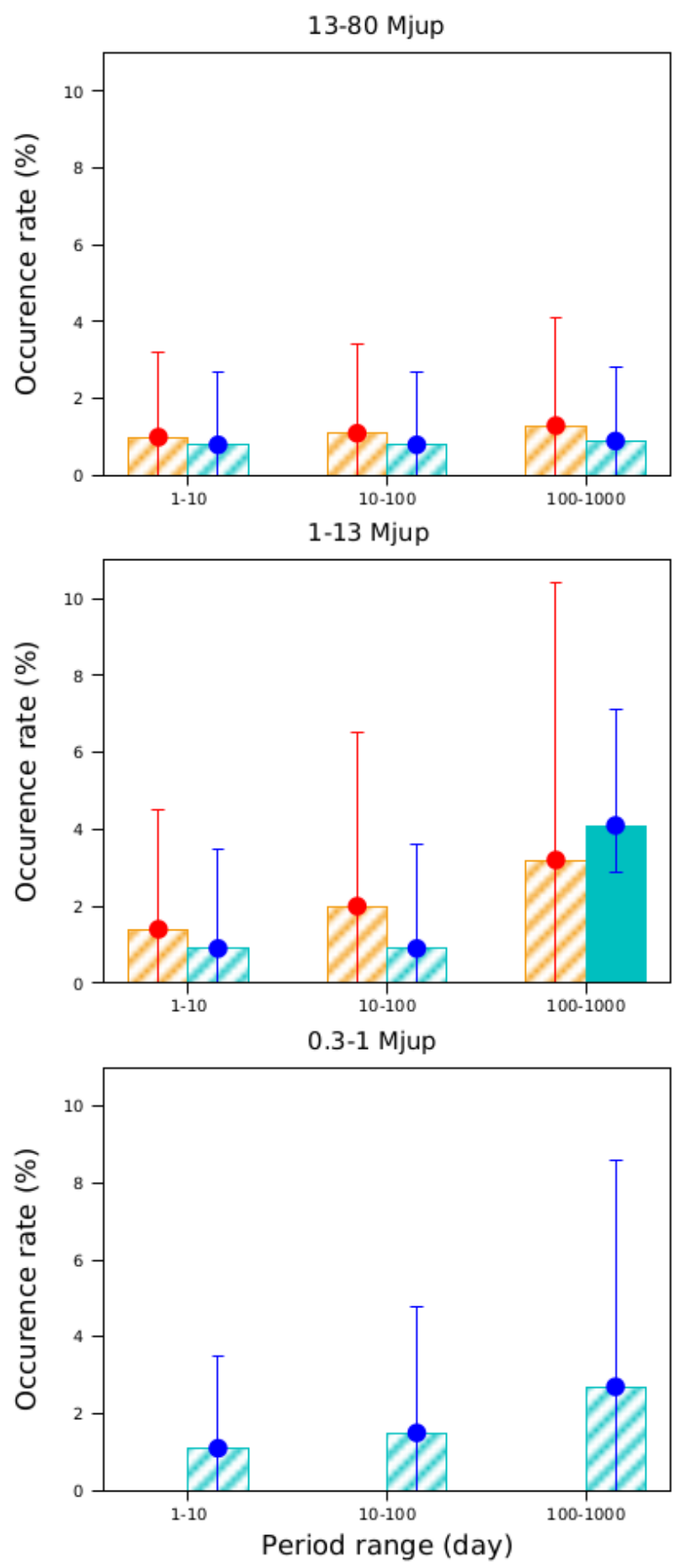

Fig. 16. Constraints on companion occurrence rates from our combined SOPHIE + HARPS survey. From top to bottom panels: brown dwarf companions (13-80 $M_{\mathrm{Jup}}$ ), Jupiter-mass companions (1-13 $\left.M_{\mathrm{Jup}}\right)$, Saturn-mass companions $\left(0.3-1 M_{\text {Jup }}\right)$. From left to right on each row: period ranges of 1-10, 10-100, and 100-1000 days. Cyan: stellar mass $\leq 1.5 M_{\odot}$ subsample (with $1 \sigma$ error bars in blue). Orange: stellar mass $>1.5 M_{\odot}$ subsample (with $1 \sigma$ error bars in red). Actual occurrence rates are displayed in full colour $\left(n_{\mathrm{det}} \geq 1\right)$ and upper limits $\left(n_{\mathrm{det}}=0\right)$ are displayed in hatch.

$P \leq 10^{3}$ days. This upper limit is two to three times lower than the upper limit we obtained in Paper IX (in agreement with a sample twice larger). Still, we cannot determine from this result whether the GP occurrence rate around A-type stars is significantly different from the value we derive for our F-type targets, or from the GP occurrence rate provided by Cumming et al. (2008) for FGK dwarf stars. We can only say that the occurrence rate of super-Jupiter GPs does not rise steeply for A-type dwarfs compared to FGK dwarfs for $P \leq 10^{3}$ days. Given the remaining uncertainty, it is still compatible with the increased global GP frequency versus stellar mass predicted by the coreaccretion theory (Kennedy \& Kenyon 2008, see also below).

3 . Finally, we consider Saturn-mass $\left(0.3 \leq m_{\mathrm{p}} \sin i \leq 1 M_{\text {Jup }}\right)$ companions around our lower mass targets $\left(\leq 1.5 M_{\odot}\right)$. We do not detect any such companions in our SOPHIE survey, which was already the case for our HARPS survey (Paper IX). With a completeness between 75\% (1 $\leq P \leq 10$ days $)$ and $40 \%\left(10^{2} \leq P \leq 10^{3}\right.$ days $)$, we constrain the occurrence rate to be $\leq 3.5$ and $\leq 8.6 \%(1 \sigma)$, respectively. These upper limits are roughly two times lower than those we obtained in Paper IX for the HARPS survey only.

Companion frequency versus period. For BD-mass companions, our completeness is above $90 \%$ for all the considered period ranges between 1 and $10^{3}$ days; thus, there is no really significant difference between the occurrence rate values we get for these period ranges.

In the case of planetary-mass companions, five out of the six GPs we detected (with the exception of candidate HD 113337c with its longer period of $\sim 3260$ days) belong to a somewhat limited $\left(P, m_{\mathrm{p}} \sin i\right)$ domain, with orbital periods between 200 and 1000 days and minimal masses between 1 and $10 M_{\text {Jup }}$. Our sensitivity to companions in this domain ranges from $20-30 \%$ to 70-80\%; we achieve a better completeness closer to the star, yet we do not detect any GPs for these smaller periods. We note that SAFIR preliminary tests based on ELODIE RV were shown to be able to re-detect a known $P \sim 3$-day hot Jupiter (Galland et al. 2005a) and to detect a $P \sim 28$-day BD (Galland et al. 2006). Thus, this apparent concentration of GPs at the largest considered period ranges is most probably not induced by a bias in our method.

In terms of companion frequency, we constrain the hot Jupiter $\left(P<10\right.$ or $<10^{2}$ days) occurrence rate to be below $\leq 3 \%$ for our targets $\leq 1.5 M_{\odot}$ and to be below $\leq 4.5-6.5 \%$ for our targets $>1.5 M_{\odot}$. These upper limits on the occurrence rates and their $1 \sigma$ uncertainties are a factor of $\sim 2$ lower compared to Paper IX. In the case of our low-mass targets, this hotJupiter upper limit is smaller than (but still compatible with) the $4.1_{-1.2}^{+3} \%$ value we get in the $10^{2}-10^{3}$-day range (where most of our detected GPs are located). This result is compatible with a possible increase in the GP frequency with the orbital period, at least for our low-mass subsample. Based on the core-accretion theory, Kennedy \& Kenyon (2009) predicted that GPs would be found at longer periods around more massive stars. That we do not detect any GPs around our high-mass targets in the 1- to $10^{3}$-day range could be in agreement with such a trend, although it remains speculative as long as we are not able to constrain the occurrence rate of GPs on wider $(\sim 3-5 \mathrm{au})$ orbits around A-type stars (with the help of direct imaging surveys and/or Gaia).

Since we do not detect any hot Jupiters in our sample, we only have an upper limit on the hot-Jupiter frequency, i.e. $\leq 3$ $4.5 \%(1 \sigma)$. Then, we cannot tell if there is a difference with the hot-Jupiter rates of $0.5-1.5 \%$ derived for FGK MS stars (Santerne et al. 2016). Since several hot Jupiters have recently been detected by transits and Doppler tomography around AF stars (see e.g. Bourrier et al. 2015; Stevens et al. 2017; Crouzet et al. 2017; McLeod et al. 2017; Zhou et al. 2017), it would be very interesting to compare our constraints on the hot-Jupiter 
frequencies around AF stars with similar statistics derived from transit survey results.

Comparison to other stars. The GP occurrence rate $\left(3.7_{-1.1}^{+2.8} \%\right)$ we derive for our F-type $\left(1-1.5 M_{\odot}\right)$ subsample shows no significant difference when compared to solar-like FGK stars. However, our upper limit on the GP occurrence rate $(\leq 6.3 \%$ at $1 \sigma)$ around A-type stars $\left(1.5-2.5 M_{\odot}\right)$ is significantly smaller than both the GP frequencies around GK subgiants $(11 \pm 2 \%)$ and giants $\left(\simeq 15_{-3}^{+8} \%\right)$ derived in approximately the same period ranges by Johnson et al. (2010) and Reffert et al. (2015), respectively. Since we compute our detection limits only for circular orbits (which is the common approach; see Paper IX for details), the uncertainties on our companion occurrence rates are slightly underestimated by not considering a variable companion eccentricity, and might still be compatible with the above results. Still, this discrepancy in the GP occurrence rate between our A-type subsample and the evolved star samples of Johnson et al. (2010) and Reffert et al. (2015) remains significant. A possible interpretation is that different stellar properties (e.g. stellar mass, metallicity) significantly impact the formation and evolution of giant planets (see e.g. Ghezzi et al. 2018).

Finally, it is also interesting to compare the companion frequency around AF MS stars to the companion population around white dwarfs (WDs), as the latter typically have AF-type progenitors. Based on searches for an infrared excess in the WD spectral energy distribution, Farihi et al. (2005) and Girven et al. (2011) estimated the global BD occurrence rate around WDs to be $\lesssim 1 \%$; this estimation should be robust, as WD are ideal targets to search for low-mass stellar and substellar companions, due to their low luminosity. This result is consistent with the overall 3-4\% upper limit we obtain on the BD occurrence rate in the 1 - to $10^{3}$-day period range. It is also in agreement with the absence of BD detections within 2 au of the primary reported by Jones et al. (2017) for subgiant stars. We note that our upper limit for the considered periods $\left(P=10^{3}\right.$ days, or sma $\left.\simeq 2.5-3 \mathrm{au}\right)$ roughly corresponds to the closest orbits for which a substellar companion is expected to avoid engulfment during the red giant transition (Mustill \& Villaver 2012; Nordhaus \& Spiegel 2013).

\section{Conclusion}

We have carried out a systematic RV search for BD and GP companions around 125 northern AF MS dwarfs with SOPHIE. This survey has led to the detection of one GP (or possibly two) around the F6V dwarf HD 113337 and does not contradict the existence of the already reported GP around HD 16232. We additionally detected fourteen high-mass and/or distant companions based on high-amplitude RV variations or long-term RV trends, of which 12 are probably of stellar nature, based either on $\mathrm{CCF}$ and/or FWHM variations or independent detection from the literature. We finally detected one (possibly two) faint SB2 binary based on a strong RV-FWHM correlation and complex low-amplitude RV variations (HD 191195 and hypothetically HD 185395).

We combined this SOPHIE survey to the HARPS twin survey detailed in Paper IX to conduct a global statistical study of the BD/GP occurrence rate within $\sim 2-2.5$ au around AF MS stars. We were able to derive good constraints on these occurrence rates, with a BD occurrence rate below 3-4\% and a GP ( $1<m_{\mathrm{p}} \sin i<13 M_{\text {Jup }}$ ) occurrence rate below 3-5\% for periods below 100 days and of $\simeq 4_{-1}^{+3} \%$ for periods in the $10^{2}-10^{3}$ day range and stellar masses below $1.5 M_{\odot}$. Our BD occurrence rates are compatible with the BD frequencies reported both for FGK dwarfs and for evolved GK subgiants and giants. Our GP occurrence rates for stars with masses in the 1-1.5 $M_{\odot}$ range are compatible with the GP frequencies reported for FGK dwarfs. However, our upper limits on the GP frequency around stars with masses in the 1.5-2.5 $M_{\odot}$ range are somewhat smaller than the GP frequencies reported for evolved GK stars, which are considered to be their descendants.

The fact that we do not detect any GPs around our A-type targets (compared to the six GPs detected or reported for our F-type targets) is compatible with the prediction that GPs form further away from the primary and migrate less with an increasing stellar mass, though it does not confirm it. Our results can be of interest for planetary population synthesis studies (see e.g. Mordasini et al. 2009, 2012), especially when considering the influence of the stellar mass on the planetary formation and migration processes. In this context, the astrometry from Gaia will probe with an unprecedented sensitivity the GP population at a few au around A-type stars in the solar neighbourhood, thus allowing a promising synergy with our RV survey (Sozzetti 2015; Sahlmann et al. 2016).

Finally, we did not detect any hot Jupiter in our 225-target sample, constraining their occurrence rate to be below $3-5 \%$ if considering $\mathrm{A}$ and $\mathrm{F}$ stars separately. While this result does not show a significant difference with the results reported for solar-mass stars, it would be of great interest to compare it to a statistical study of the hot Jupiters that have recently been detected by transits around more distant AF stars.

Acknowledgements. We acknowledge support from the French CNRS and from the Agence Nationale de la Recherche (ANR grant GIPSE ANR-14-CE33-0018). N.C.S. acknowledges support by Fundação para a Ciência e a Tecnologia (FCT, Portugal) through national funds and by FEDER through COMPETE2020 by grants UID/FIS/04434/2013 \& POCI-01-0145-FEDER-007672 and PTDC/FISAST/1526/2014 \& POCI-01-0145-FEDER-016886, and through Investigador FCT contract no. IF/00169/2012/CP0150/CT0002. These results have made use of the SIMBAD database, operated at the CDS, Strasbourg, France. We would like to thank B. Gaensick and J. Farihi for their useful suggestions on the companion occurrence rate of white dwarfs as the descendants of AF MS stars. We would like to thank our anonymous referee for the useful additional suggestions.

\section{References}

Allen, C., Poveda, A., \& Herrera, M. A. 2000, A\&A, 356, 529

Allende Prieto, C., \& Lambert, D. L. 1999, A\&A, 352, 555

Baliunas, S. L., Donahue, R. A., Soon, W. H., et al. 1995, ApJ, 438, 269

Baraffe, I., Chabrier, G., Allard, F., \& Hauschildt, P. H. 1998, A\&A, 337, 403

Baranne, A., Queloz, D., Mayor, M., et al. 1996, A\&AS, 119, 373

Boesgaard, A. M., \& Tripicco, M. J. 1986, ApJ, 303, 724

Boisse, I., Bouchy, F., Hébrard, G., et al. 2011, Eur. Phys. J. Web Conf., 11, 02005

Bonfils, X., Delfosse, X., Udry, S., et al. 2013, A\&A, 549, A109

Borgniet, S., Boisse, I., Lagrange, A.-M., et al. 2014, A\&A, 561, A65

Borgniet, S., Meunier, N., \& Lagrange, A.-M. 2015, A\&A, 581, A133

Borgniet, S., Lagrange, A.-M., Meunier, N., \& Galland, F. 2017, A\&A, 599, A57

Borucki, W. J., Koch, D., Basri, G., et al. 2010, Science, 327, 977

Bouchy, F., \& Sophie Team. 2006, in Tenth Anniversary of 51 Peg-b: Status of and Prospects for Hot Jupiter Studies, eds. L. Arnold, F. Bouchy, \& C. Moutou (Paris: Frontier Group), 319

Bouchy, F., Pepe, F., \& Queloz, D. 2001, A\&A, 374, 733

Bouchy, F., Hébrard, G., Udry, S., et al. 2009, A\&A, 505, 853

Bouchy, F., Díaz, R. F., Hébrard, G., et al. 2013, A\&A, 549, A49

Bouchy, F., Ségransan, D., Díaz, R. F., et al. 2016, A\&A, 585, A46

Bourrier, V., Lecavelier des Etangs, A., Hébrard, G., et al. 2015, A\&A, 579, A55

Casagrande, L., Schönrich, R., Asplund, M., et al. 2011, A\&A, 530, A138

Chelli, A. 2000, A\&A, 358, L59

Crouzet, N., McCullough, P. R., Long, D., et al. 2017, AJ, 153, 94

Cumming, A., Butler, R. P., Marcy, G. W., et al. 2008, PASP, 120, 531

De Rosa, R. J., Patience, J., Wilson, P. A., et al. 2014, MNRAS, 437, 1216

Desort, M., Lagrange, A.-M., Galland, F., Udry, S., \& Mayor, M. 2007, A\&A, 473,983 
S. Borgniet et al.: Extrasolar planets and brown dwarfs around AF-type stars. X.

Desort, M., Lagrange, A.-M., Galland, F., et al. 2008, A\&A, 491, 883 Desort, M., Lagrange, A.-M., Galland, F., et al. 2009, A\&A, 506, 1469

Díaz, R. F., Santerne, A., Sahlmann, J., et al. 2012, A\&A, 538, A113

Dumusque, X., Lovis, C., Ségransan, D., et al. 2011, A\&A, 535, A55

Dumusque, X., Boisse, I., \& Santos, N. C. 2014, ApJ, 796, 132

Endl, M., Brugamyer, E. J., Cochran, W. D., et al. 2016, ApJ, 818, 34

Farihi, J., Becklin, E. E., \& Zuckerman, B. 2005, ApJS, 161, 394

Fischer, D. A., \& Valenti, J. 2005, ApJ, 622, 1102

Frankowski, A., Jancart, S., \& Jorissen, A. 2007, A\&A, 464, 377

Fressin, F., Torres, G., Charbonneau, D., et al. 2013, ApJ, 766, 81

Galland, F., Lagrange, A.-M., Udry, S., et al. 2005a, A\&A, 444, L21

Galland, F., Lagrange, A.-M., Udry, S., et al. 2005b, A\&A, 443, 337

Galland, F., Lagrange, A.-M., Udry, S., et al. 2006, A\&A, 452, 709

Ghezzi, L., Montet, B. T., \& Johnson, J. A. 2018, ApJ, 860, 109

Girven, J., Gänsicke, B. T., Steeghs, D., \& Koester, D. 2011, MNRAS, 417, 1210

Goldin, A., \& Makarov, V. V. 2007, ApJS, 173, 137

Grether, D., \& Lineweaver, C. H. 2006, ApJ, 640, 1051

Grieves, N., Ge, J., Thomas, N., et al. 2017, MNRAS, 467, 4264

Griffin, R. F. 2010, The Observatory, 130, 17

Guenther, E. W., Hartmann, M., Esposito, M., et al. 2009, A\&A, 507, 1659

Gullikson, K., Endl, M., Cochran, W. D., \& MacQueen, P. J. 2015, ApJ, 815, 62

Guzik, J. A., Houdek, G., Chaplin, W. J., et al. 2011, ArXiv e-prints [arXiv: 1110.2120 ]

Hartkopf, W. I., Tokovinin, A., \& Mason, B. D. 2012, AJ, 143, 42

Hartmann, M., \& Hatzes, A. P. 2015, A\&A, 582, A84

Howard, A. W., \& Fulton, B. J. 2016, PASP, 128, 114401

Howard, A., Marcy, G., Johnson, J. A., et al. 2011, BAAS, 43, 415.06

Johnson, J. A., Aller, K. M., Howard, A. W., \& Crepp, J. R. 2010, PASP, 122, 905

Jones, M. I., Brahm, R., Wittenmyer, R. A., et al. 2017, A\&A, 602, A58

Kane, S. R., Barclay, T., Hartmann, M., et al. 2015, ApJ, 815, 32

Kennedy, G. M., \& Kenyon, S. J. 2008, ApJ, 673, 502

Kennedy, G. M., \& Kenyon, S. J. 2009, ApJ, 695, 1210

Kiyaeva, O. V., Kiselev, A. A., \& Izmailov, I. S. 2008, Astron. Lett., 34, 405

Lagrange, A.-M., Desort, M., Galland, F., Udry, S., \& Mayor, M. 2009, A\&A, 495,335

Ligi, R., Mourard, D., Lagrange, A. M., et al. 2012, A\&A, 545, A5

Makarov, V. V., \& Kaplan, G. H. 2005, AJ, 129, 2420

Malkov, O. Y., Tamazian, V. S., Docobo, J. A., \& Chulkov, D. A. 2012, A\&A, 546, A69

Mayor, M., Lovis, C., \& Santos, N. C. 2014, Nature, 513, 328

McLeod, K. K., Rodriguez, J. E., Oelkers, R. J., et al. 2017, AJ, 153, 263

Meunier, N., Desort, M., \& Lagrange, A.-M. 2010, A\&A, 512, A39
Meunier, N., Lagrange, A.-M., \& De Bondt K. 2012, A\&A, 545, A87

Monnier, J. D., Berger, J.-P., Millan-Gabet, R., \& ten Brummelaar T. A. 2004, in New Frontiers in Stellar Interferometry, ed. W. A. Traub, Proc. SPIE, 5491, 1370

Mordasini, C., Alibert, Y., Benz, W., \& Naef, D. 2009, A\&A, 501, 1161

Mordasini, C., Alibert, Y., Benz, W., Klahr, H., \& Henning, T. 2012, A\&A, 541, A97

Mustill, A. J., \& Villaver, E. 2012, ApJ, 761, 121

Nordhaus, J., \& Spiegel, D. S. 2013, MNRAS, 432, 500

Pepe, F., Mayor, M., Rupprecht, G., et al. 2002, The Messenger, 110, 9

Pourbaix, D., Ivezić, Ž., Knapp, G. R., Gunn, J. E., \& Lupton, R. H. 2004, A\&A, 423, 755

Press, W. H., \& Rybicki, G. B. 1989, ApJ, 338, 277

Queloz, D., Henry, G. W., Sivan, J. P., et al. 2001, A\&A, 379, 279

Reffert, S., Bergmann, C., Quirrenbach, A., Trifonov, T., \& Künstler, A. 2015, A\&A, 574, A116

Rhee, J. H., Song, I., Zuckerman, B., \& McElwain, M. 2007, ApJ, 660, 1556

Riddle, R. L., Tokovinin, A., Mason, B. D., et al. 2015, ApJ, 799, 4

Roberts, D. H., Lehar, J., \& Dreher, J. W. 1987, AJ, 93, 968

Roberts, Jr. L. C., Tokovinin, A., Mason, B. D., et al. 2015, AJ, 149, 118

Rodriguez, D. R., \& Zuckerman, B. 2012, ApJ, 745, 147

Sahlmann, J., Ségransan, D., Queloz, D., et al. 2011, A\&A, 525, A95

Sahlmann, J., Martín-Fleitas, J., Mora, A., et al. 2016, in Space Telescopes and Instrumentation 2016: Optical, Infrared, and Millimeter Wave, Proc. SPIE, 9904, 99042E

Santerne, A., Díaz, R. F., Almenara, J.-M., et al. 2015, MNRAS, 451, 2337

Santerne, A., Moutou, C., Tsantaki, M., et al. 2016, A\&A, 587, A64

Santos, N. C., Israelian, G., \& Mayor, M. 2004, A\&A, 415, 1153

Santos, N. C., Mortier, A., Faria, J. P., et al. 2014, A\&A, 566, A35

Scargle, J. D. 1982, ApJ, 263, 835

Schneider, J., Dedieu, C., Le Sidaner, P., Savalle, R., \& Zolotukhin, I. 2011, A\&A, 532, A79

Schröder, K.-P., Mittag, M., Hempelmann, A., González-Pérez, J. N., \& Schmitt, J. H. M. M. 2013, A\&A, 554, A50

Ségransan, D., Mayor, M., Udry, S., et al. 2011, A\&A, 535, A54

Sozzetti, A. 2015, ArXiv e-prints [arXiv:1502 .03575]

Stevens, D. J., Collins, K. A., Gaudi, B. S., et al. 2017, AJ, 153, 178

Tokovinin, A. 2014, AJ, 147, 86

Toyota, E., Itoh, Y., Ishiguma, S., et al. 2009, PASJ, 61, 19 van Leeuwen F. 2007, A\&A, 474, 653

White, T. R., Huber, D., Maestro, V., et al. 2013, MNRAS, 433, 1262

Wright, J. T. 2016, ArXiv e-prints [arXiv:1611.05398]

Zhou, G., Bakos, G. Á., Hartman, J. D., et al. 2017, AJ, 153, 211 


\section{Appendix A: SOPHIE sample}

The main physical properties and the main characteristics of the spectroscopic observables for each of the targets of our SOPHIE sample are detailed in Table A.1.

Table A.1. Stellar characteristics and detailed results for the 125 targets of our SOPHIE RV survey.

\begin{tabular}{|c|c|c|c|c|c|c|c|c|c|c|c|c|c|c|c|c|c|}
\hline & & tellar cha & acteristi & & & & & & & Sur & detaile & d result & & & & & \\
\hline$\overline{\mathrm{HD}}$ & HIP & ST & $B-V$ & $v \sin i$ & Mass & TBL & $N_{\mathrm{m}}$ & & $\mathrm{RV}$ & & & BIS & & & $\langle F W\rangle$ & $\mathrm{V}$ & $\overline{\mathrm{CL}}$ \\
\hline & & & & & & & & $A$ & $\mathrm{rms}$ & $\langle U\rangle$ & $A$ & rms & $\langle U\rangle$ & corr. & & & \\
\hline & & & & $\mathrm{km} \mathrm{s}^{-1}$ & $M_{\odot}$ & day & & & $\mathrm{m} \mathrm{s}^{-1}$ & & & $\mathrm{~ms} \mathrm{~s}^{-1}$ & & & $\mathrm{~km} \mathrm{~s}^{-1}$ & & \\
\hline 400 & 699 & F8IV & 0.456 & 3 & 1.15 & 1503 & 34 & 40.6 & 9.8 & 5.6 & 29.9 & 7.7 & 6.3 & -0.37 & 11.1 & $\mathrm{C}$ & \\
\hline 1404 & 1473 & $\mathrm{~A} 2 \mathrm{~V}$ & 0.05 & 110 & 2.21 & 1134 & 16 & 1280 & 338 & 259 & & & & & & $\mathrm{C}$ & \\
\hline 3268 & 2832 & F7V & 0.52 & 5.8 & 1.37 & 1493 & 21 & 36.1 & 10.7 & 6.4 & 22.9 & 5.4 & 9.9 & 0.02 & 10.7 & $\mathrm{C}$ & \\
\hline 3440 & 3132 & F6V & 0.501 & 4.7 & 1.19 & 173 & 17 & 32.2 & 7.9 & 5.5 & 23.9 & 5.2 & 5.5 & -0.12 & 9.4 & $\mathrm{C}$ & \\
\hline 6288 & 4979 & A8IV & 0.243 & 94 & 1.76 & 361 & 16 & 743 & 219 & 82.1 & 6114 & 1350 & 205 & -0.17 & 134 & V & \\
\hline 6961 & 5542 & A7V & 0.18 & 91 & 1.8 & 1417 & 14 & 198 & 46.1 & 48.4 & & & & & & $\mathrm{C}$ & \\
\hline 7193 & 5631 & F5V & 0.49 & 8.2 & 1.05 & 357 & 16 & 67.3 & 22.3 & 6.6 & 63.7 & 20.6 & 10.8 & -0.66 & 14.3 & V & \\
\hline 8723 & 6706 & $\mathrm{~F} 2 \mathrm{~V}$ & 0.343 & 60 & 1.38 & 1553 & 25 & 263 & 65.7 & 38.8 & 5293 & 1167 & 96.1 & -0.37 & 88.6 & $\mathrm{C}$ & \\
\hline 8829 & 6748 & F0.5V & 0.283 & 18 & 1.49 & 102 & 16 & 251 & 89.6 & 12.5 & 123 & 28.9 & 28.6 & -0.4 & 28.8 & V & \\
\hline 8907 & 6878 & F8V & 0.49 & 9.5 & 1.23 & 395 & 19 & 219 & 55.5 & 8.3 & 118 & 34.1 & 16.3 & -0.56 & 20.7 & $\mathrm{~V}$ & \\
\hline 9780 & 7447 & FOIV & 0.24 & 113 & 1.8 & 823 & 13 & 888 & 265 & 102 & 6261 & 2129 & 255 & 0.58 & 177 & V & \\
\hline 10453 & 7916 & F5V & 0.44 & 13 & 1.49 & 1589 & 17 & $\begin{array}{c}2244 \\
71.9\end{array}$ & $\begin{array}{l}660 \\
21.6\end{array}$ & 10.9 & 19133 & 4783 & 23.9 & 0.92 & 22.8 & $\begin{array}{l}\mathrm{V} \\
\mathrm{C}\end{array}$ & $a$ \\
\hline 11973 & 9153 & FOV & 0.271 & 97 & 1.77 & 648 & 21 & 926 & 236 & 59.9 & 3876 & 1173 & 149 & -0.2 & 138 & $\mathrm{~V}$ & \\
\hline 12111 & 9480 & $\mathrm{~A} 3 \mathrm{~V}$ & 0.16 & 69 & 1.97 & 1399 & 14 & 202 & 48.8 & 35.5 & 3015 & 927 & 87.8 & 0.32 & 86.5 & $\mathrm{C}$ & \\
\hline 13555 & 10306 & F5V & 0.4 & 9 & 1.45 & 679 & 39 & 80.7 & 21.1 & 6.1 & 104 & 25 & 8.4 & 0.05 & 13.5 & $\mathrm{~V}$ & \\
\hline 16232 & 12184 & F6V & 0.5 & 42 & 1.2 & 1892 & 27 & $\begin{array}{l}568 \\
262\end{array}$ & $\begin{array}{c}161 \\
72.5\end{array}$ & 21 & 700 & 160 & 50.9 & -0.08 & 60.7 & $\begin{array}{l}\text { V } \\
\text { V }\end{array}$ & $a$ \\
\hline 16765 & 12530 & F7V & 0.52 & 32 & 1.2 & 318 & 30 & 276 & 60.1 & 27.6 & 556 & 129 & 67.8 & -0.51 & 48.2 & V & \\
\hline 16895 & 12777 & F8V & 0.51 & 9 & 1.15 & 687 & 23 & 62.4 & 16.5 & 7.2 & 39 & 10 & 12.8 & 0.33 & 14.9 & $\mathrm{~V}$ & \\
\hline 17948 & 13665 & F5V & 0.381 & 6.1 & 1.33 & 2102 & 24 & 62.1 & 12.5 & 6.6 & 45.9 & 11.4 & 10.5 & -0.3 & 13.5 & $\mathrm{C}$ & \\
\hline 18404 & 13834 & F5IV & 0.41 & 26 & 1.35 & 1360 & 14 & 110 & 28.8 & 11.9 & 586 & 198 & 27 & -0.73 & 37.7 & V & \\
\hline 20395 & 15244 & F5V & 0.364 & 6 & 1.36 & 1175 & 22 & $\begin{array}{c}6444 \\
46.3\end{array}$ & $\begin{array}{c}2669 \\
14.5\end{array}$ & 6.6 & 587 & 238 & 10.6 & -0.97 & 13 & $\begin{array}{l}\text { V } \\
\text { V }\end{array}$ & $a$ \\
\hline 20677 & 15648 & $\mathrm{~A} 3 \mathrm{~V}$ & 0.07 & 130 & 2 & 8 & 22 & 1445 & 299 & 178 & & & & & & $\mathrm{C}$ & \\
\hline 25490 & 18907 & $\mathrm{~A} 0.5 \mathrm{~V}$ & 0.045 & 65 & 2.27 & 1817 & 14 & 741 & 197 & 131 & & & & & & $\mathrm{C}$ & \\
\hline 25621 & 18993 & F6V & 0.5 & 15 & 1.45 & 1433 & 15 & $\begin{array}{l}65.5 \\
51.8\end{array}$ & $\begin{array}{l}21.1 \\
14.5\end{array}$ & 7.8 & 137 & 32.8 & 14.9 & $\begin{array}{l}-0.73 \\
0\end{array}$ & 25.5 & $\begin{array}{l}\mathrm{V} \\
\mathrm{C}\end{array}$ & $b$ \\
\hline 27819 & 20542 & $\mathrm{~A} 2 \mathrm{~V}$ & 0.15 & 45 & 1.96 & 1399 & 25 & 633 & 182 & 17.5 & 943 & 247 & 41.9 & -0.05 & 75.3 & $\mathrm{~V}$ & \\
\hline 27934 & 20635 & A7IV-V & 0.143 & 87 & 2.07 & 1883 & 18 & 1323 & 327 & 43.2 & & & & & & $\mathrm{~V}$ & \\
\hline 27946 & 20641 & A7V & 0.231 & 175 & 1.82 & 1750 & 17 & 1104 & 27 & 254 & & & & & & $\mathrm{C}$ & \\
\hline 27962 & 20648 & A2IV-V & 0.049 & 8.3 & 2.29 & 1009 & 70 & 78. & 19 & 6.4 & 91 & 14 & 10 & 0.09 & 18 & V & \\
\hline 28355 & 20901 & $\mathrm{~A} 5 \mathrm{~V}$ & 0.212 & 93 & 1.91 & 1535 & 20 & 408 & 89 & 42.7 & 36564 & 6306 & 106 & 0.36 & 115 & $\mathrm{~V}$ & \\
\hline 29488 & 21683 & A5V & 0.157 & 115 & 2.04 & 1370 & 26 & 1085 & 271 & 79.2 & & & & & & V & \\
\hline 31662 & 23380 & F4V & 0.391 & 28 & 1.44 & 2160 & 33 & 120 & 29.5 & 13 & 64 & 167 & 29.9 & -0.22 & 42.5 & $\mathrm{~V}$ & \\
\hline 31675 & 23484 & F6V & 0.48 & 8.8 & 1.23 & 645 & 32 & 110 & 31.4 & 6.6 & 150 & 32.7 & 10.6 & -0.51 & 19.2 & V & \\
\hline 33608 & 24162 & F5V & 0.423 & 14 & 1.43 & 2216 & 14 & $\begin{array}{c}116 \\
68.2\end{array}$ & $\begin{array}{l}32.4 \\
17.4\end{array}$ & 8 & 195 & 67 & 15.5 & $\begin{array}{l}-0.84 \\
0\end{array}$ & 22.9 & $\begin{array}{l}\mathrm{V} \\
\mathrm{V}\end{array}$ & $b$ \\
\hline 43042 & 29650 & F5IV-V & 0.44 & 10 & 1.33 & 1018 & 49 & 65.8 & 13.6 & 7.2 & 40 & 7.5 & 12.9 & -0.01 & 11.2 & $\mathrm{C}$ & \\
\hline 43318 & 29716 & F5V & 0.5 & 4.6 & 1.4 & 1850 & 21 & $\begin{array}{l}33.7 \\
20.8\end{array}$ & $\begin{array}{c}10.2 \\
5.2\end{array}$ & 5.8 & 32.8 & 8.1 & 7.2 & 0.42 & 10.6 & $\begin{array}{l}\mathrm{C} \\
\mathrm{C}\end{array}$ & $a$ \\
\hline 43386 & 29800 & F5V & 0.42 & 18 & 1.33 & 2247 & 21 & 90.3 & 23.8 & 9.8 & 258 & 66.3 & 21 & -0.55 & 29.9 & V & \\
\hline 48737 & 32362 & F5IV-V & 0.43 & 68 & 1.63 & 1448 & 13 & 123 & 37.1 & 28.5 & 1163 & 393 & 70.1 & -0.15 & 92 & $\mathrm{C}$ & \\
\hline 58461 & 35998 & F5V & 0.42 & 14 & 1.49 & 1861 & 22 & 371 & 114 & 10.1 & 837 & 252 & 21.6 & -0.93 & 24.4 & $\mathrm{~V}$ & $b$ \\
\hline
\end{tabular}

Notes. Spectral type (ST) and $B-V$ values and $v \sin i$ values are taken from the CDS database. Stellar masses are taken from Allende Prieto \& Lambert (1999). The survey results include the observation time baseline (TBL), the number of computed spectra $N_{\mathrm{m}}$, the peak-to-peak (i.e. maximum-to-minimum) amplitude $A$, rms and mean uncertainty $\langle U\rangle$ on the RV and BIS measurements, the RV-BIS correlation (Pearson's coefficient), and the mean FWHM $(\langle\mathrm{FW}\rangle)$. V stands for our RV variability criterion (Paper IX), with V for RV variable stars and C for $\mathrm{RV}$ constant targets. The "CL" column reports the correction applied on the RV, if any: ${ }^{(a)}$ correction from binary or planetary fit; ${ }^{(b)}$ correction from RV-BIS activity correlation. 
Table A.1. continued.

\begin{tabular}{|c|c|c|c|c|c|c|c|c|c|c|c|c|c|c|c|c|c|}
\hline & & tellar char & cteristic & & & & & & & & ey detail & $\mathrm{d}$ results & & & & & \\
\hline HD & HIP & ST & $B-V$ & $v \sin i$ & Mass & TBL & $N_{\mathrm{m}}$ & & $\mathrm{RV}$ & & & BIS & & RV- & $\langle F W\rangle$ & $\mathrm{V}$ & $\overline{\mathrm{CL}}$ \\
\hline & & & & & & & & $A$ & $\mathrm{rms}$ & $\langle U\rangle$ & A & $\mathrm{rms}$ & $\langle U\rangle$ & corr. & & & \\
\hline & & & & $\mathrm{km} \mathrm{s}^{-1}$ & $M_{\odot}$ & day & & & $\mathrm{m} \mathrm{s}^{-1}$ & & & $\mathrm{~m} \mathrm{~s}^{-1}$ & & & $\mathrm{~km} \mathrm{~s}^{-1}$ & & \\
\hline & & & & & & & & 186 & 42.3 & & & & & 0 & & $\mathrm{~V}$ & \\
\hline 58855 & 36439 & F6V & 0.414 & 10 & 1.25 & 793 & 69 & 49.7 & 10.6 & 5.9 & 55.1 & 10 & 7.8 & -0.02 & 14.9 & $\mathrm{C}$ & \\
\hline 58946 & 36366 & F1V & 0.32 & 63 & 1.5 & 456 & 18 & 372 & 86.7 & 35.8 & & & & & & V & \\
\hline 63332 & 38325 & F6V & 0.441 & 7 & 1.13 & 1136 & 24 & 63.2 & 15 & 6.1 & 52.9 & 13.4 & 8.7 & 0.31 & 14.7 & V & \\
\hline 69548 & 40875 & F4V & 0.367 & 55 & 1.34 & 1500 & 24 & 298 & 62.9 & 29.1 & 1923 & 462 & 71.5 & -0.33 & 76.2 & V & \\
\hline 69897 & 40843 & F6V & 0.51 & 5 & 1.25 & 1193 & 27 & 56.9 & 13.5 & 5.7 & 38.8 & 9.2 & 6.8 & 0.03 & 10.9 & $\mathrm{~V}$ & \\
\hline 70313 & 41152 & $\mathrm{~A} 3 \mathrm{~V}$ & 0.12 & 100 & 1.83 & 1004 & 13 & 650 & 221 & 143 & 77613 & 20230 & 358 & -0.15 & 169 & $\mathrm{C}$ & \\
\hline 75332 & 43410 & F7V & 0.498 & 10 & 1.17 & 3412 & 68 & 125 & 33.4 & 6.1 & 95.5 & 20.6 & 8.6 & -0.45 & 15.2 & $\mathrm{~V}$ & \\
\hline 75616 & 43625 & F5V & 0.42 & 7.5 & 1.04 & 826 & 37 & 149 & 35.3 & 7 & 114 & 31.5 & 12.3 & -0.49 & 16.5 & V & \\
\hline 76398 & 43932 & A7IV & 0.161 & 119 & 1.98 & 2246 & 28 & 503 & 143 & 85 & 77355 & 14173 & 212 & -0.1 & 147 & $\mathrm{C}$ & \\
\hline 76582 & 44001 & FOIV & 0.207 & 92 & 1.71 & 754 & 16 & 457 & 123 & 64.9 & 9508 & 2930 & 162 & -0.08 & 130 & $\mathrm{C}$ & \\
\hline 76644 & 44127 & A7V & 0.19 & 129 & 1.64 & 1163 & 20 & 1470 & 443 & 84 & 35467 & 8604 & 210 & 0.19 & 188 & $\mathrm{~V}$ & \\
\hline 78154 & 45038 & F7V & 0.49 & 5.4 & 1.43 & 765 & 82 & 49.1 & 10.4 & 5.4 & 33 & 6.2 & 5.2 & -0.01 & 12 & $\mathrm{C}$ & \\
\hline 79439 & 45493 & $\mathrm{~A} 6 \mathrm{~V}$ & 0.175 & 145 & 1.89 & 742 & 34 & 1348 & 331 & 135 & & & & & & V & \\
\hline 80290 & 45836 & F3V & 0.42 & 4.5 & 1.14 & 2670 & 40 & 40.2 & 9.6 & 5.8 & 32.2 & 7.4 & 7.4 & -0.25 & 10.6 & $\mathrm{C}$ & \\
\hline 82328 & 46853 & F7V & 0.46 & 6.3 & 1.53 & 3367 & 75 & $\begin{array}{l}102 \\
66.5\end{array}$ & $\begin{array}{c}17 \\
11.7\end{array}$ & 5.7 & 106 & 13.3 & 14.3 & 0.04 & 13.9 & $\begin{array}{l}\text { V } \\
\text { V }\end{array}$ & $a$ \\
\hline 89449 & 50564 & F6IV-V & 0.44 & 16 & 1.44 & 3375 & 47 & 112 & 25.1 & 9.1 & 372 & 75.1 & 22.6 & -0.43 & 25.4 & V & \\
\hline 90277 & 51056 & F0V & 0.242 & 33 & 2.1 & 1171 & 30 & 169 & 38 & 12.7 & 800 & 168 & 29.1 & -0.24 & 50.8 & $\mathrm{~V}$ & \\
\hline 90470 & 51200 & $\mathrm{~A} 3 \mathrm{~V}$ & 0.138 & 105 & 1.89 & 2157 & 24 & 2215 & 463 & 222 & 24058 & 5268 & 556 & 0.06 & 187 & V & \\
\hline 91312 & 51658 & A7IV & 0.197 & 128 & 1.83 & 2634 & 34 & $\begin{array}{l}5202 \\
1809\end{array}$ & $\begin{array}{c}1724 \\
417\end{array}$ & 91.5 & & & & & & $\begin{array}{l}\text { V } \\
\text { V }\end{array}$ & $a$ \\
\hline 95418 & 53910 & A1IV & -0.0 & 35 & 2.28 & 2619 & 28 & 187 & 47.3 & 28 & & & & & & $\mathrm{C}$ & \\
\hline 97855 & 55044 & F6V & 0.413 & 5.7 & 1.32 & 2619 & 23 & 41 & 11.8 & 6.5 & 34.9 & 9.6 & 10.4 & -0.42 & 12.8 & $\mathrm{C}$ & \\
\hline 99747 & 56035 & F5V & 0.39 & 8.4 & 1.41 & 1255 & 36 & 49.2 & 11 & 10.1 & 102 & 20.9 & 21.6 & 0.14 & 18.1 & $\mathrm{C}$ & \\
\hline 102647 & 57632 & A3V & 0.09 & 115 & 1.9 & 1145 & 102 & 700 & 134 & 111 & & & & & & $\mathrm{C}$ & \\
\hline 102870 & 57757 & F9V & 0.55 & 4.4 & 1.36 & 3370 & 87 & 54 & 11.2 & 5.4 & 27 & 5.8 & 13.4 & 0.08 & 10.2 & V & \\
\hline 106591 & 59774 & $\mathrm{~A} 2 \mathrm{~V}$ & 0.09 & 251 & 2.1 & 1990 & 21 & 1962 & 52 & 498 & & & & & & $\mathrm{C}$ & \\
\hline 107113 & 59879 & F4V & 0.43 & 5.8 & 1.32 & 1154 & 36 & 71.8 & 16.1 & 6.6 & 52.3 & 14 & 10.5 & -0.06 & 12.7 & $\mathrm{~V}$ & \\
\hline 109141 & 61212 & $\mathrm{~F} 2 \mathrm{~V}$ & 0.338 & 142 & 1.46 & 1392 & 16 & 845 & 279 & 152 & 8004 & 2462 & 380 & 0.87 & 195 & $\mathrm{C}$ & \\
\hline 111270 & 62402 & A9V & 0.2 & 93 & 1.89 & 3309 & 24 & 1031 & 223 & 84.9 & & & & & & $\mathrm{~V}$ & \\
\hline 113337 & 63584 & F6V & 0.372 & 6 & 1.41 & 3368 & 301 & $\begin{array}{l}328 \\
132\end{array}$ & $\begin{array}{l}68.1 \\
22.1\end{array}$ & 7.9 & 148 & 4.4 & 19.8 & -0.32 & 13.7 & $\begin{array}{l}\text { V } \\
\text { V }\end{array}$ & $a$ \\
\hline 115810 & 64979 & A7IV & 0.24 & 98 & 1.78 & 1433 & 27 & 739 & 169 & 83.9 & 8411 & 1766 & 209 & -0.14 & 145 & V & \\
\hline 118232 & 66234 & $\mathrm{~A} 4 \mathrm{~V}$ & 0.12 & 145 & 2.06 & 1435 & 19 & 1285 & 330 & 116 & 115938 & 25992 & 290 & -0.17 & 221 & $\mathrm{~V}$ & \\
\hline 119992 & 67103 & F7IV-V & 0.47 & 6.2 & 1.25 & 2621 & 53 & 49 & 12.2 & 11.5 & 61 & 11.6 & 28.8 & -0.14 & 13.8 & $\mathrm{C}$ & \\
\hline 121164 & 67782 & A8IV & 0.187 & 65 & 1.9 & 3371 & 60 & 492 & 121 & 37.3 & & & & & & $\mathrm{~V}$ & \\
\hline 121560 & 68030 & F6V & 0.56 & 5 & 1 & 2680 & 27 & 40.9 & 10.6 & 7.6 & 27.4 & 6.2 & 19 & 0.11 & 9.9 & $\mathrm{C}$ & \\
\hline 124675 & 69483 & A7IV & 0.23 & 111 & 1.93 & 107 & 17 & 2448 & 810 & 77.5 & 36187 & 10492 & 193 & -0.35 & 174 & V & \\
\hline 125040 & 69751 & F8V & 0.49 & 36 & 1.16 & 2677 & 17 & 171 & 45.1 & 21.6 & 437 & 133 & 54.1 & -0.11 & 52.9 & $\mathrm{~V}$ & \\
\hline 125451 & 69989 & F5IV & 0.364 & 40 & 1.4 & 1476 & 18 & 16 & 43 & 21.9 & 6 & 17 & 53.3 & -0.63 & 58.5 & $\mathrm{C}$ & \\
\hline 126141 & 70310 & F5V & 0.358 & 7 & 1.36 & 2680 & 14 & 112 & 41.7 & 8.6 & 139 & 38.2 & 21.4 & -0.67 & 15.1 & $\mathrm{~V}$ & \\
\hline 129153 & 71759 & F0V & 0.216 & 105 & 1.68 & 48 & 22 & 1805 & 439 & 109 & 16573 & 3043 & 272 & -0.17 & 159 & $\mathrm{~V}$ & \\
\hline 130044 & 72066 & F0V & 0.261 & 15 & 1.55 & 519 & 36 & $\begin{array}{l}245 \\
178\end{array}$ & $\begin{array}{l}63.9 \\
45.5\end{array}$ & 9.5 & 338 & 99.8 & 20.1 & $\begin{array}{l}-0.7 \\
0\end{array}$ & 24.1 & $\begin{array}{l}\text { V } \\
\text { V }\end{array}$ & \\
\hline 132254 & 73100 & F8V & 0.53 & 5 & 1.29 & 1462 & 19 & 34 & 9.3 & 6 & 29.8 & 9 & 8.2 & -0.3 & 14 & $\mathrm{C}$ & \\
\hline 132375 & 73309 & F8V & 0.5 & 6.2 & 1.38 & 1520 & 14 & 25.3 & 9.3 & 6 & 37.3 & 9.4 & 8.1 & -0.44 & 13.7 & $\mathrm{C}$ & \\
\hline 134083 & 73996 & F5V & 0.43 & 45 & 1.33 & 1505 & 20 & 110 & 28.4 & 19.8 & 372 & 85.6 & 47.7 & -0.23 & 64 & $\mathrm{C}$ & \\
\hline 136729 & 75043 & $\mathrm{~A} 4 \mathrm{~V}$ & 0.115 & 145 & 2.05 & 2956 & 32 & 1517 & 373 & 144 & & & & & & $\mathrm{~V}$ & \\
\hline 137391 & 75411 & FOIV & 0.291 & 89 & 1.8 & 668 & 21 & 1154 & 322 & 44.6 & 16935 & 3174 & 111 & 0.35 & 124 & V & \\
\hline 139389 & 76456 & F5V & 0.4 & 21 & 1.29 & 1224 & 16 & 287 & 68.3 & 14.7 & 177 & 49.7 & 34.6 & 0.57 & 34.1 & V & \\
\hline 142860 & 78072 & F6IV & 0.5 & 10 & 1.28 & 2071 & 23 & 49.8 & 14.2 & 6.2 & 69.4 & 15.5 & 8.8 & -0.02 & 17.1 & $\mathrm{~V}$ & \\
\hline 143584 & 78286 & FOIV & 0.258 & 68 & 1.58 & 2595 & 18 & $\begin{array}{l}2137 \\
1180\end{array}$ & $\begin{array}{l}575 \\
291\end{array}$ & 75.8 & 2123 & 634 & 190 & -0.21 & 103 & $\begin{array}{l}\text { V } \\
\text { V }\end{array}$ & \\
\hline
\end{tabular}


Table A.1. continued.

\begin{tabular}{|c|c|c|c|c|c|c|c|c|c|c|c|c|c|c|c|c|c|}
\hline \multicolumn{6}{|c|}{ Stellar characteristics } & \multicolumn{12}{|c|}{ Survey detailed results } \\
\hline \multirow[t]{3}{*}{ HD } & \multirow[t]{3}{*}{ HIP } & \multirow[t]{3}{*}{ ST } & \multirow[t]{3}{*}{$B-V$} & \multirow{3}{*}{$\begin{array}{c}v \sin i \\
\mathrm{~km} \mathrm{~s}^{-1}\end{array}$} & \multirow{3}{*}{$\begin{array}{c}\text { Mass } \\
\\
M_{\odot}\end{array}$} & \multirow{3}{*}{$\begin{array}{l}\text { TBL } \\
\text { day } \\
\end{array}$} & \multirow[t]{3}{*}{$N_{\mathrm{m}}$} & \multicolumn{3}{|c|}{$\mathrm{RV}$} & \multicolumn{3}{|c|}{ BIS } & \multirow{3}{*}{$\begin{array}{l}\text { RV- } \\
\text { BIS } \\
\text { corr. }\end{array}$} & \multirow{3}{*}{$\begin{array}{l}\langle F W\rangle \\
\mathrm{km} \mathrm{s}^{-1}\end{array}$} & \multirow[t]{3}{*}{$\mathrm{V}$} & \multirow[t]{3}{*}{$\overline{C L}$} \\
\hline & & & & & & & & $A$ & $\mathrm{rms}$ & $\langle U\rangle$ & $A$ & $\mathrm{rms}$ & $\langle U\rangle$ & & & & \\
\hline & & & & & & & & \multicolumn{3}{|c|}{$\mathrm{m} \mathrm{s}^{-1}$} & \multicolumn{3}{|c|}{$\mathrm{m} \mathrm{s}^{-1}$} & & & & \\
\hline 147365 & 80008 & F4V & 0.4 & 77 & 1.38 & 2252 & 14 & 357 & 102 & 37.5 & 7755 & 1685 & 93 & -0.05 & 104 & $\mathrm{~V}$ & \\
\hline 148048 & 79822 & F5V & 0.37 & 80 & 1.52 & 2987 & 46 & $\begin{array}{l}1678 \\
272\end{array}$ & $\begin{array}{c}571 \\
71.2\end{array}$ & 56.5 & 9907 & 1582 & 141 & -0.16 & 125 & $\begin{array}{l}\text { V } \\
\text { C }\end{array}$ & $a$ \\
\hline 149681 & 80480 & FoV & 0.24 & 118 & 1.62 & 2891 & 27 & 1942 & 417 & 119 & 6499 & 1707 & 297 & -0.29 & 176 & V & \\
\hline 152303 & 81854 & F4V & 0.41 & 22 & 1.32 & 1803 & 24 & 278 & 73.7 & 13.2 & 531 & 138 & 30.4 & -0.6 & 36.1 & V & \\
\hline 154431 & 83494 & A6V & 0.19 & 110 & 1.67 & 3195 & 23 & 561 & 150 & 109 & & & & & & $\mathrm{C}$ & \\
\hline 156295 & 84183 & A7V & 0.192 & 103 & 1.66 & 3157 & 20 & 1184 & 331 & 80.3 & & & & & & V & \\
\hline 159332 & 85912 & F4V & 0.45 & 7.2 & 1.41 & 3285 & 29 & 48.9 & 10.9 & 8.3 & 54.9 & 15.1 & 20.7 & -0.26 & 15.7 & $\mathrm{C}$ & \\
\hline 162003 & 86614 & F5IV-V & 0.44 & 9 & 1.48 & 2890 & 22 & $\begin{array}{c}1806 \\
126\end{array}$ & $\begin{array}{c}524 \\
34.4\end{array}$ & 8.6 & 492 & 124 & 21.5 & -0.28 & 19.2 & $\begin{array}{l}\text { V } \\
\text { V }\end{array}$ & $a$ \\
\hline 168151 & 89348 & F5V & 0.38 & 10 & 1.45 & 3197 & 23 & 79.5 & 19.5 & 11.5 & 89.3 & 18.1 & 28.7 & -0.46 & 14.4 & $\mathrm{C}$ & \\
\hline 184960 & 96258 & F7V & 0.51 & 6.5 & 1.28 & 1900 & 29 & 66.4 & 14.8 & 6.1 & 44.9 & 9.9 & 8.7 & -0.45 & 14.1 & V & \\
\hline 185395 & 96441 & F3V & 0.38 & 10 & 1.37 & 3482 & 326 & $\begin{array}{l}405 \\
288\end{array}$ & $\begin{array}{l}82.3 \\
65.2\end{array}$ & 7.5 & 82.4 & 12.5 & 18.6 & 0.02 & 11.1 & $\begin{array}{l}\text { V } \\
\text { V }\end{array}$ & $a$ \\
\hline 186689 & 97229 & A3IV & 0.18 & 33 & 1.71 & 3193 & 71 & $\begin{array}{l}513 \\
270\end{array}$ & $\begin{array}{c}101 \\
59.4\end{array}$ & 17.9 & 1738 & 358 & 44.7 & $\begin{array}{l}-0.81 \\
0\end{array}$ & 50 & $\begin{array}{l}\text { V } \\
\text { V }\end{array}$ & $b$ \\
\hline 187013 & 97295 & F5IV-V & 0.47 & 7.3 & 1.21 & 3234 & 27 & 92.1 & 17.7 & 8 & 81.8 & 18.9 & 20 & 0.15 & 16 & V & \\
\hline 191195 & 99026 & F5V & 0.39 & 5.5 & 1.49 & 3191 & 265 & 272 & 56.4 & 7.6 & 98 & 17.6 & 18.9 & 0.2 & 12.4 & V & \\
\hline 192985 & 99889 & F5V & 0.391 & 8.7 & 1.44 & 3236 & 19 & 94 & 22.3 & 10.6 & 167 & 41.6 & 26.6 & -0.5 & 18.6 & V & \\
\hline 193369 & 100108 & $\mathrm{~A} 2 \mathrm{~V}$ & 0.06 & 90 & 1.99 & 2795 & 37 & 1167 & 280 & 185 & & & & & & $\mathrm{C}$ & \\
\hline 196524 & 101769 & F5IV & 0.44 & 48 & 1.92 & 1178 & 19 & $\begin{array}{c}2075 \\
197\end{array}$ & $\begin{array}{c}601 \\
45\end{array}$ & 18.3 & 934 & 269 & 43.8 & -0.52 & 62.8 & $\begin{array}{l}\text { V } \\
\text { V }\end{array}$ & $a$ \\
\hline 197373 & 102011 & F6IV & 0.399 & 27 & 1.34 & 387 & 23 & 262 & 61.5 & 12.9 & 303 & 91.9 & 29.7 & -0.56 & 42.9 & V & \\
\hline 197950 & 102253 & A8V & 0.21 & 160 & 1.67 & 783 & 21 & 1598 & 428 & 399 & & & & & & $\mathrm{C}$ & \\
\hline 198390 & 102805 & F5V & 0.395 & 6.5 & 1.21 & 773 & 25 & 35.5 & 9.6 & 7.3 & 70.1 & 15.5 & 13.3 & 0.28 & 14.2 & $\mathrm{C}$ & \\
\hline 199254 & 103298 & $\mathrm{~A} 5 \mathrm{~V}$ & 0.11 & 145 & 2.02 & 1382 & 15 & $\begin{array}{l}1371 \\
597\end{array}$ & $\begin{array}{l}340 \\
158\end{array}$ & 151 & & & & & & $\begin{array}{l}\text { V } \\
\text { C }\end{array}$ & $a$ \\
\hline 204153 & 105769 & F0V & 0.32 & 115 & 1.49 & 340 & 15 & 814 & 255 & 88.3 & 3254 & 748 & 220 & 0.34 & 164 & V & \\
\hline 204414 & 105966 & A1V & 0.046 & 70 & 2 & 748 & 19 & 459 & 113 & 72.3 & 26815 & 5247 & 180 & 0.28 & 124 & $\mathrm{C}$ & \\
\hline 206677 & 107302 & A7IV-V & 0.204 & 113 & 1.67 & 1414 & 20 & 1412 & 338 & 92 & 8022 & 1992 & 230 & -0.5 & 168 & V & \\
\hline 209369 & 108535 & F5V & 0.409 & 24 & 1.62 & 1056 & 24 & $\begin{array}{l}369 \\
229\end{array}$ & $\begin{array}{l}93.8 \\
62.5\end{array}$ & 11.7 & 728 & 204 & 26.2 & $\begin{array}{l}-0.75 \\
0\end{array}$ & 39.9 & $\begin{array}{l}\text { V } \\
\text { V }\end{array}$ & $b$ \\
\hline 210715 & 109521 & A5V & 0.142 & 130 & 1.95 & 1879 & 45 & $\begin{array}{l}2152 \\
1363\end{array}$ & $\begin{array}{l}609 \\
376\end{array}$ & 111 & 105313 & 17072 & 277 & -0.05 & 185 & $\begin{array}{l}\text { V } \\
\text { V }\end{array}$ & $a$ \\
\hline 211976 & 110341 & F5V & 0.421 & 5 & 1.3 & 323 & 70 & 64.5 & 13 & 5.9 & 35.9 & 8.1 & 7.6 & -0.15 & 12.5 & V & \\
\hline 212754 & 110785 & F7V & 0.52 & 6.8 & 1.37 & 67 & 19 & $\begin{array}{l}2083 \\
39.4\end{array}$ & $\begin{array}{l}508 \\
11.8\end{array}$ & 5.8 & 56.7 & 16.4 & 7.3 & 0.16 & 14.9 & $\begin{array}{l}\text { V } \\
\text { V }\end{array}$ & $a$ \\
\hline 214454 & 111674 & A9V & 0.24 & 96 & 2.02 & 1331 & 15 & 395 & 133 & 42.2 & 2256 & 717 & 105 & -0.19 & 150 & V & \\
\hline 215588 & 112324 & F5V & 0.388 & 13 & 1.26 & 1829 & 57 & 82.6 & 19.5 & 7.8 & 132 & 27.7 & 14.9 & -0.27 & 22.1 & V & \\
\hline 215648 & 112447 & F6V & 0.49 & 9 & 1.34 & 769 & 18 & 45.4 & 12.7 & 6.1 & 30.4 & 8.4 & 8.7 & -0.16 & 14 & V & \\
\hline 216385 & 112935 & F6V & 0.48 & 4.7 & 1.48 & 760 & 74 & 76.9 & 13.9 & 5.5 & 40.3 & 7.7 & 5.8 & -0.34 & 10.9 & V & \\
\hline 218261 & 114096 & F6V & 0.49 & 6 & 1.16 & 509 & 65 & 82 & 15.3 & 5.9 & 79.2 & 12.1 & 7.5 & -0.29 & 13.4 & V & \\
\hline 218396 & 114189 & F0V & 0.257 & 49. & 1.42 & 808 & 111 & 4508 & 1072 & 36.4 & & & & & & V & \\
\hline 218470 & 114210 & F5V & 0.44 & 8.8 & 1.48 & 2213 & 27 & 93.1 & 26.2 & 6.9 & 221 & 51.5 & 11.6 & 0.23 & 18.8 & V & \\
\hline 220974 & 115770 & A6IV & 0.16 & 98 & 1.99 & 1592 & 27 & 200 & 61 & 52.1 & 28092 & 4746 & 130 & 0.22 & 153 & $\mathrm{C}$ & \\
\hline 222368 & 116771 & F7V & 0.5 & 7 & 1.38 & 3158 & 38 & 83.2 & 19.1 & 5.4 & 25.9 & 6.3 & 4.9 & 0.3 & 12.3 & V & \\
\hline 222603 & 116928 & A7V & 0.21 & 60 & 1.88 & 788 & 22 & 1556 & 380 & 26.3 & 4069 & 1336 & 64.5 & -0.49 & 85.5 & V & \\
\hline 223731 & 117681 & F5V & 0.44 & 31 & 1.33 & 778 & 21 & 188 & 48.5 & 17.7 & 500 & 151 & 42.5 & -0.21 & 46.9 & V & \\
\hline
\end{tabular}

\section{Appendix B: Sample selection}

We selected the targets in our sample to try to keep it as representative as possible of AF MS dwarfs more massive than the Sun, while making it appropriate for accurate RV measurements with SAFIR. In terms of spectral type, we selected stars between $\mathrm{A} 0$ and $\sim \mathrm{F} 8$ types. The A0 limit corresponds roughly to the earliest spectral type for which close (sma $\lesssim 2.5 \mathrm{au}$ ) GP and/or BD companions are detectable with SAFIR RV, while the
F7-F9 limit roughly corresponds to the earliest spectral types for which the traditional cross-correlation technique with a binary mask gives accurate RV measurements. In terms of distance to the Sun ( $d$, taken from the HipPARCos catalogue), we set two cutoffs in order to obtain a statistically significant sample of nearby stars and to keep roughly the same proportion of A- and F-type targets: $d \leq 56$ and $d \leq 33$ parsecs for A- and F-type stars, respectively. To keep only objects of dwarf nature, we selected stars with an absolute magnitude 
within 2.5 mag from the main sequence (Lagrange et al. 2009).

Then we removed confirmed spectroscopic binaries of SB2 nature, close ( $\leq 5 \operatorname{arcsec})$ visual binaries, and targets with highly variable luminosity to keep targets observable in RV. Finally, we also removed confirmed $\delta$ Scuti and $\gamma$ Doradus pulsators, and chemically peculiar Am/Ap stars that are often associated with spectroscopic binaries (Lagrange et al. 2009). This selection led to a visible dichotomy between our A- and F-type targets (as seen in Fig. 1), due to the removal of a significant number of late A and early $\mathrm{F}$ stars belonging to the instability strip. We initially ended up with $\sim 320$ stars. We then restrained our effective SOPHIE observing sample to 125 of these more than 300 targets (without any selection prior) to be able to fully cover the 1- to 1000-day periodicity space for all targets during the SOPHIE observations. These 125 targets can be divided into 72 stars with a mass $\leq 1.5 M_{\odot}$ and 53 stars with a mass $>1.5 M_{\odot}$.

\section{Appendix C: Occurrence rate computation}

For a given sample with $N$ stars, the search completeness function $C\left(P, m_{\mathrm{p}} \sin i\right)$ gives the fraction of targets for which we can rule out a planet with a period $P$ and a minimal mass $m_{\mathrm{p}} \sin i$ given the computed detection limits (see e.g. Howard et al. 2011). At a given $\left(P, m_{\mathrm{p}} \sin i\right)$ node, the completeness $C$ is defined as

$C=\frac{1}{N} \sum_{i=1}^{N} \delta_{i}$

Here, for each target $(i)$ of our sample without a detected planet, we have $\delta_{i}=1$ if the computed detection limit at this $(P$, $\left.m_{\mathrm{p}} \sin i\right)$ node is $<m_{\mathrm{p}} \sin i$ and $\delta_{i}=0$ otherwise. We compute $C\left(P, m_{\mathrm{p}} \sin i\right)$ for all the targets of our SOPHIE +HARPS combined sample without a detected planet and with more than ten spectra (219 targets overall). We compute the search completeness function using the detection limits computed from $\mathrm{RV}$ corrected from binary trends or RV-BIS correlations where appropriate.

Then, we consider a $\left(P, m_{\mathrm{p}} \sin i\right)$ domain ranging from $P_{1}$ to $P_{2}$ in periods and from $m_{\mathrm{p} 1} \sin i$ to $m_{\mathrm{p} 2} \sin i$ in minimal masses, the $\left(P, m_{\mathrm{p}} \sin i\right)$ grid being the same as used for the detection limit computation. The number of systems with detected planets within this domain is $n_{\text {det }}$ (i.e. a system with two planets within the same given domain counts as one system). To derive the search completeness of our sample over $D$, we have to sum the search completeness function $C\left(P, m_{\mathrm{p}} \sin i\right)$ over the period and the minimal mass ranges:

$$
C_{D}=\frac{\sum_{P_{1}}^{P_{2}} \sum_{m_{\mathrm{p} 1} \sin i}^{m_{\mathrm{p} 2} \sin i}\left(\frac{1}{N} \sum_{i=1}^{N} \delta_{i}\right) \mathrm{d} P \mathrm{~d}\left(m_{\mathrm{p}} \sin i\right)}{\sum_{P_{1}}^{P_{2}} \sum_{m_{\mathrm{p} 1} \sin i}^{m_{\mathrm{p} 2} \sin i} 1 \mathrm{~d} P \mathrm{~d}\left(m_{\mathrm{p}} \sin i\right)} .
$$

For a given domain $D$ with a search completeness $C_{D}$ and $n_{\text {det }}$ systems with planets, the search completeness may be far from $100 \%$. It is thus necessary to estimate the number $n_{\text {miss }}$ of potentially missed planets in order to correct for this incompleteness (Howard et al. 2011). We define $n_{\text {miss }}$ as follows:

$n_{\mathrm{miss}}=n_{\mathrm{det}} \times\left(\frac{1}{C_{D}}-1\right)$.

Now we can derive the GP occurrence rate and its $1 \sigma$ and $2 \sigma$ uncertainties. We use binomial statistics, computing the binomial distribution of $n_{\text {det }}$ GP systems among the $N$ targets. Given the probability $p$ of having one or more GPs around one given star, we compute the probability $f$ of drawing $n_{\text {det }}$ systems among the $N$ stellar targets of our sample as follows:

$f\left(n_{\mathrm{det}}, N, p\right)=\left(\begin{array}{c}N \\ n_{\mathrm{det}}\end{array}\right) \times p^{n_{\mathrm{det}}} \times(1-p)^{N-n_{\mathrm{det}}}$.

The result is the probability density function (PDF) of the GP occurrence rate (i.e. probability density versus $f$ ) in the given $\left(P, m_{\mathrm{p}} \sin i\right)$ domain. The GP occurrence rate is thus equal to the probability $f$ for which the PDF is the highest, multiplied by $\left(n_{\text {det }}+n_{\text {miss }}\right) / n_{\text {det }}$ to account for the missed GPs (see Fig. 14 in Paper IX). To derive the $1 \sigma$ and $2 \sigma$ uncertainties, we integrate the PDF and compute its standard deviations at $\sim 68$ and $\sim 95 \%$, respectively.

In the case of a domain with no detected GPs (i.e. $n_{\mathrm{det}}=0$ ), we (re-)define $n_{\text {det }}^{\prime}=1, n_{\text {miss }}=1 / C_{D}-1$, and compute the corresponding occurrence rate. The obtained value is then an upper limit on the real GP occurrence rate.

\section{Appendix D: Targets of peculiar interest: detailed plots}

Here, we display in detail the spectroscopic data of our targets either with planetary companions (HD 113337, HD 16232) or that exhibit complex RV variations (HD 185395, HD 191195). These plots include specifically the Lomb-Scargle and CLEAN periodograms of the different observables. Regarding $\theta$ Cyg, we explored in more detail the $\sim 150$-day RV periodicity to try to understand why no Keplerian model can be correctly fitted. We separated the SOPHIE RV data set over four 600-day slices (with approximately the same amount of data) and computed the corresponding Lomb-Scargle and CLEAN periodograms (Fig. D.5). We find, quite astonishingly, that the main mid-term RV periodicity is not stable at around 150 days, but decreases from $\sim 180$ to $\sim 120$ days. Furthermore, if looking at the ELODIE RV data set (which extends from BJD $-2453000 \simeq-100$ to $\simeq 1000$, i.e. before the SOPHIE observations), we find instead a periodicity of $\sim 130$ days. A similar process applied to the Howard \& Fulton (2016) RV data set (two 2000-day slices) gives a main mid-term RV periodicity of 260 or 150 days, depending on the selected time slice. We thus conclude that the observed $\theta$ Cyg $\mathrm{RV}$ variations around $\sim 150$ days are probably not really periodic, but rather quasi-periodic. This does not favour the hypothesis of a substellar companion for which the RV periodicity would have to be more stable. 

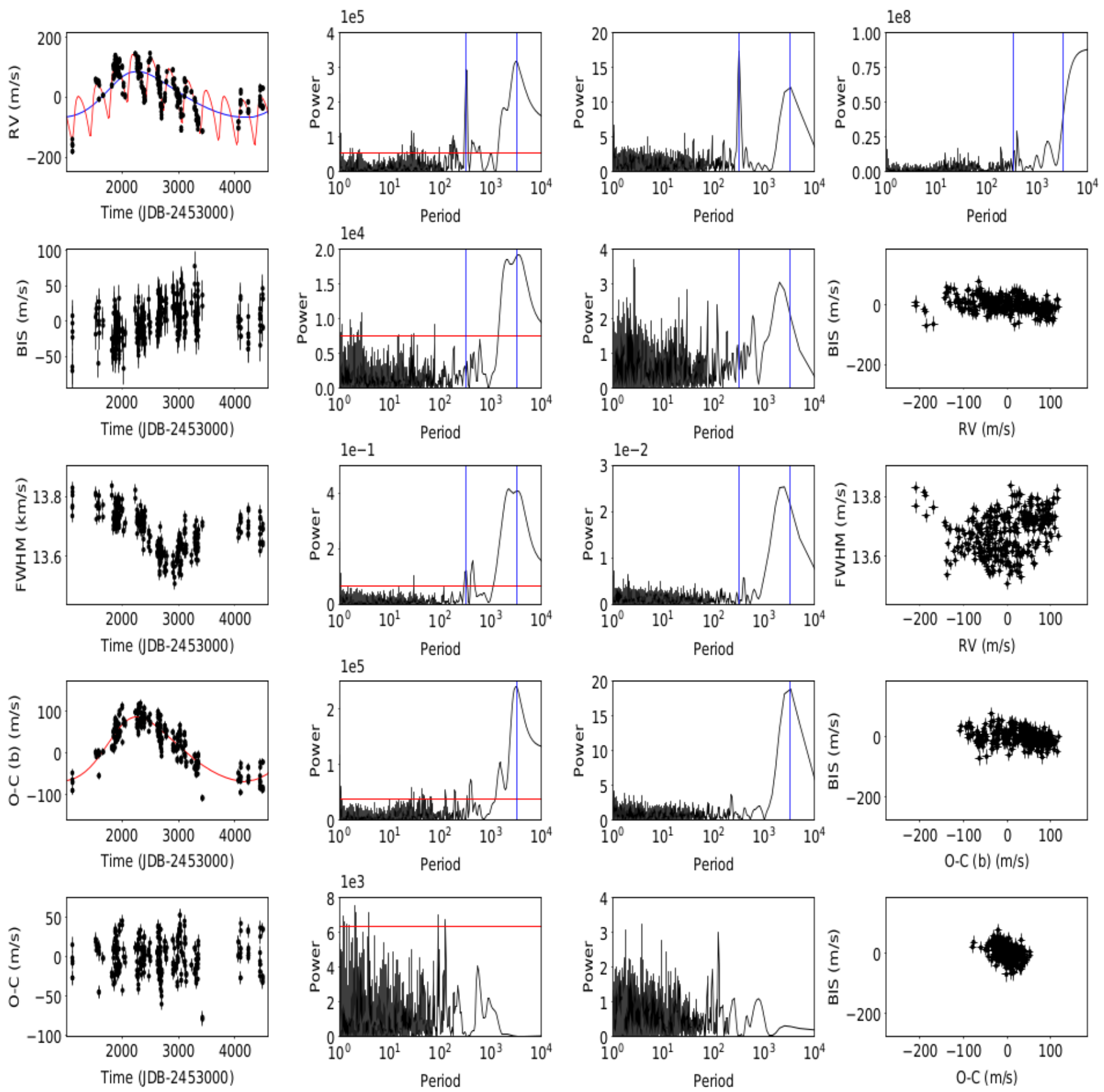

Fig. D.1. HD 113337 spectroscopic data. On all Lomb-Scargle periodograms, the $1 \%$ false-alarm probability (FAP) is plotted in red. On all periodograms, the periods of planet $\mathrm{b}$ and/or candidate planet $\mathrm{c}$ are plotted in blue. Top row, from left to right: RV time series, Lomb-Scargle RV periodogram, CLEAN RV periodogram, and observational window function. Overplotted on the RV is the two-planet Keplerian fit (red curve) and the Keplerian fit of the second candidate planet only (blue curve). Second and third rows: BIS and FWHM time series, corresponding LombScargle and CLEAN periodograms, and correlations with the RV data. Fourth row: RV residuals from planet b, corresponding Lomb-Scargle and CLEAN periodograms, and correlation with the BIS. The Keplerian fit of the candidate second planet is overplotted on the RV residuals (red curve). Fifth row: same, for the RV residuals of the two-planet Keplerian fit. 
S. Borgniet et al.: Extrasolar planets and brown dwarfs around AF-type stars. X.
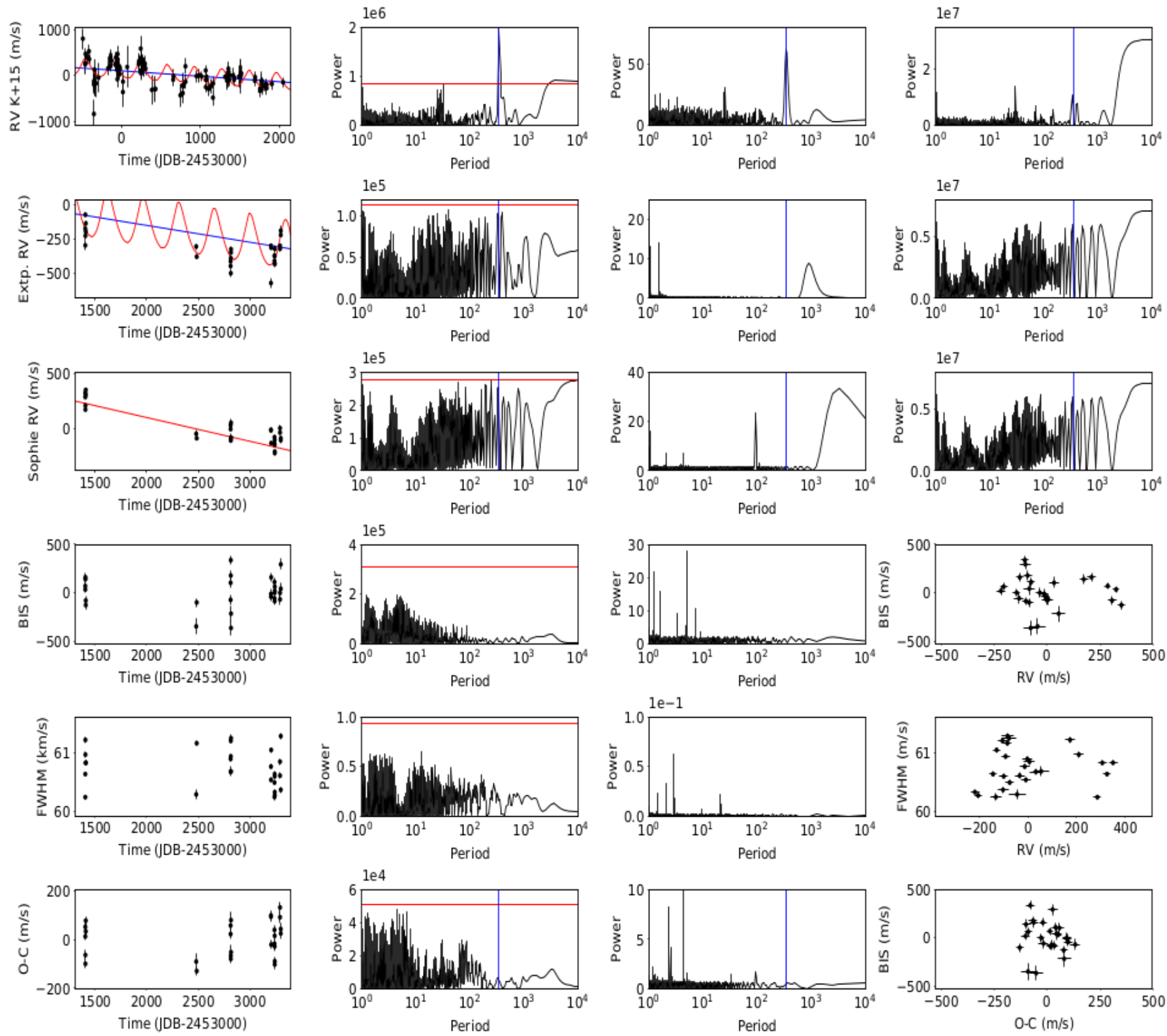

Fig. D.2. HD 16232 spectroscopic data. On the RV periodograms, HD 16232b period is overplotted in blue. First row, from left to right: HD 16232 $\mathrm{RV}$ time series from K15, corresponding Lomb-Scargle and CLEAN periodograms, and window function. Overplotted on the RV is the Keplerian fit of HD 16232b we obtained with yorbit based on the K15 data (red) and the linear drift (blue). Second row: same for the RV expected from the Keplerian+linear fit of K15 data extrapolated at the epochs of our SOPHIE observations, plus added noise. Third row: same for the SOPHIE RV time series. Overplotted on the RV is the linear fit we computed with yorbit based on the SOPHIE RV (red solid line). Each RV data set (SOPHIE and K15 data) was independently centred on 0, hence the global RV offset between the two. Fourth and fifth rows: SOPHIE BIS and FWHM time series, corresponding periodograms and correlations with SOPHIE RV. Sixth row: residuals from the linear fit to the SOPHIE RV, corresponding periodograms and correlation to SOPHIE BIS. 

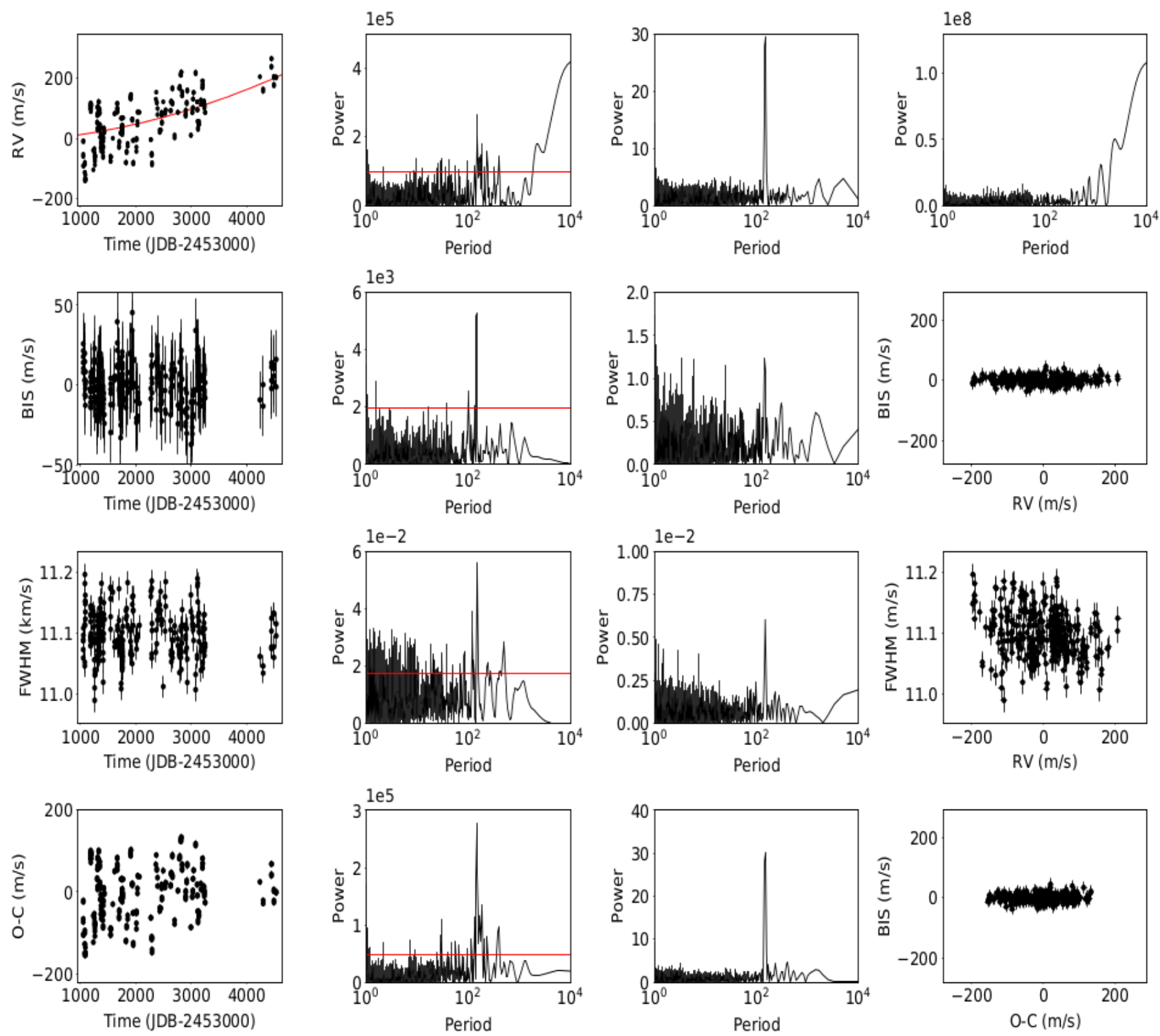

Fig. D.3. $\theta$ Cyg spectroscopic data. Top row, from left to right: RV time series, Lomb-Scargle and CLEAN periodograms of the RV, and window function of the observations. The RV long-term quadratic trend is overplotted on the RV (red solid curve). Second and third rows: BIS and FWHM time series, corresponding Lomb-Scargle and CLEAN periodograms, and correlations with the RV. Fourth row: RV residuals from the quadratic fit, Lomb-Scargle and CLEAN periodograms of the RV residuals, and correlation of the RV residuals with the BIS. 
S. Borgniet et al.: Extrasolar planets and brown dwarfs around AF-type stars. X.
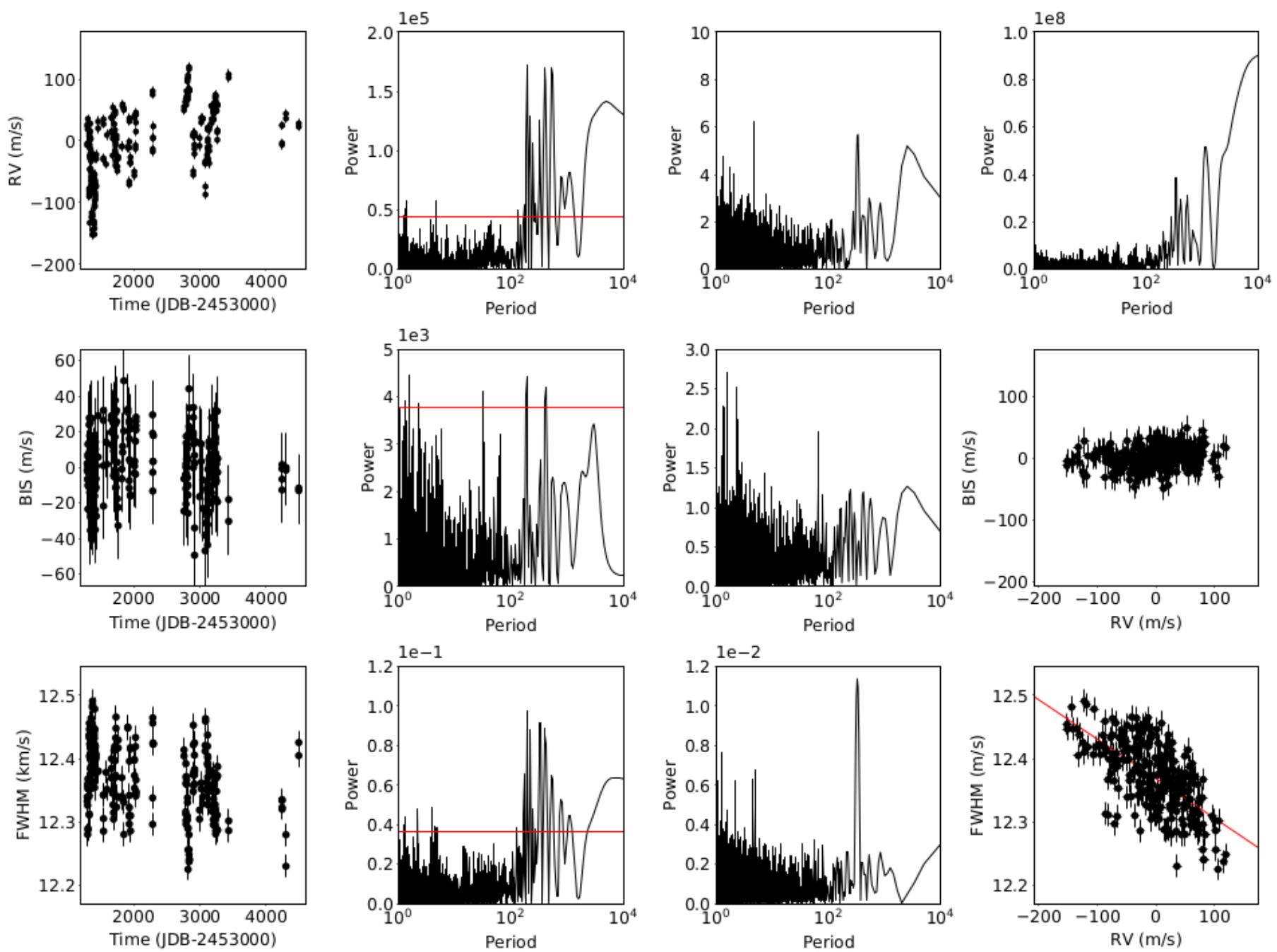

Fig. D.4. HD 191195 spectroscopic data. Top row, from left to right: RV time series, Lomb-Scargle and CLEAN periodograms of the RV, and window function of the observations. Second and third rows: BIS and FWHM time series, corresponding Lomb-Scargle and CLEAN periodograms, and correlations with the RV. The RV-FWHM correlation is overplotted in red. 

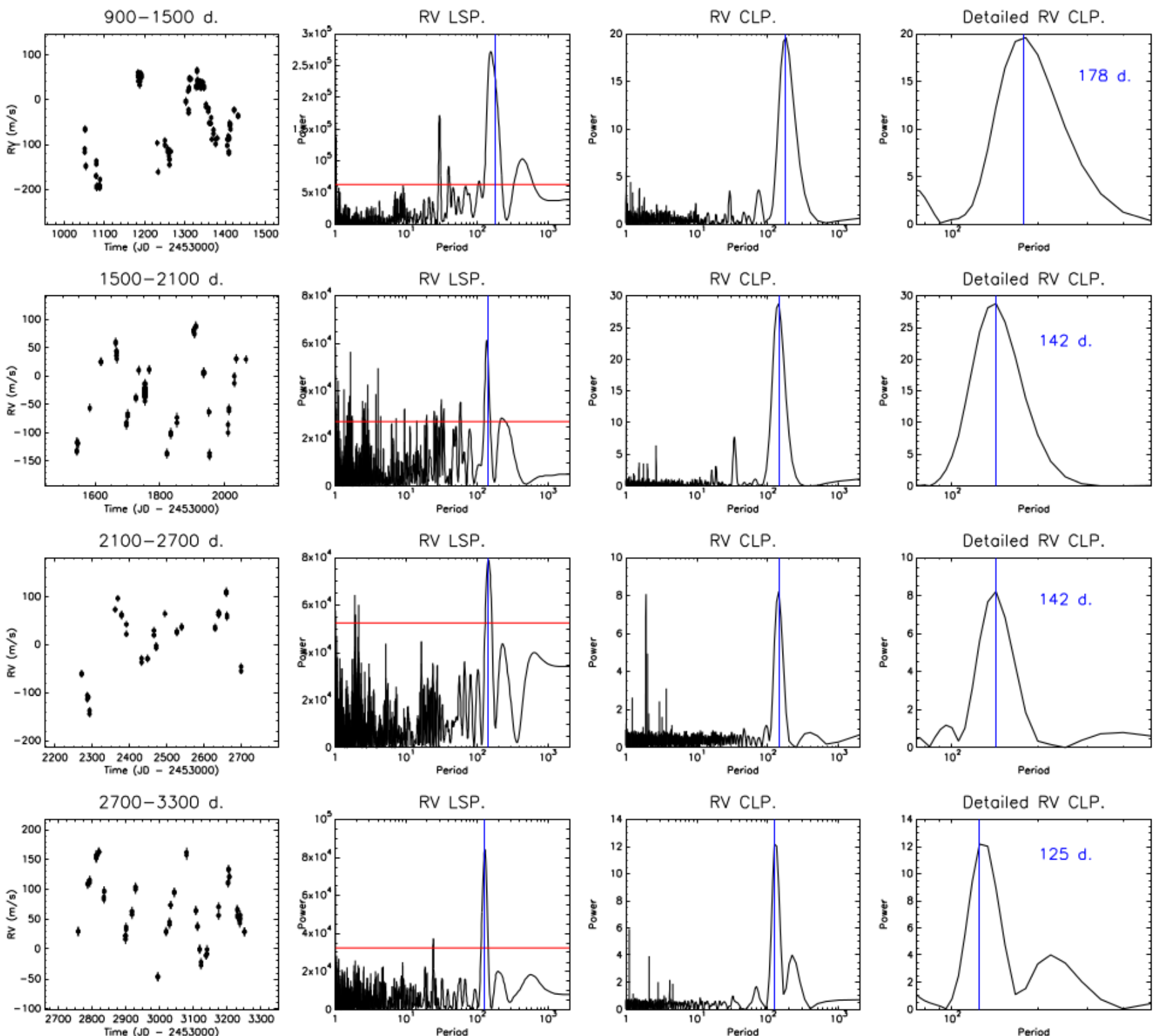

Fig. D.5. Detail of $\theta$ Cyg SOPHIE RV variations (from top to bottom rows) over four 600-day slices. From left to right columns: RV variations over the time slice, RV Lomb-Scargle periodogram (with 1\% FAP in red), RV CLEAN periodogram, detail of the CLEAN periodogram. For each time slice, the main RV periodicity deduced from the CLEAN periodogram is overplotted in red. 